\title{
Asymptotic M5-brane entropy from S-duality
}

\author{
Seok Kim and June Nahmgoong \\ Department of Physics and Astronomy \& Center for Theoretical Physics, \\ Seoul National University, \\ 1 Gwanak-ro, Gwanak-gu, Seoul 08826, Korea \\ E-mail: skim@phya.snu.ac.kr, earendi125@snu.ac.kr
}

Abstract: We study M5-branes compactified on $S^{1}$ from the D0-D4 Witten index in the Coulomb phase. We first show that the prepotential of this index is S-dual, up to a simple anomalous part. This is an extension of the well-known S-duality of the $4 \mathrm{~d} \mathcal{N}=4$ theory to the $6 \mathrm{~d}(2,0)$ theory on finite $T^{2}$. Using this anomalous S-duality, we find that the asymptotic free energy scales like $N^{3}$ when various temperature-like parameters are large. This shows that the number of 5d Kaluza-Klein fields for light D0-brane bound states is proportional to $N^{3}$. We also compute some part of the asymptotic free energy from $6 \mathrm{~d}$ chiral anomalies, which precisely agrees with our D0-D4 calculus.

Keywords: Duality in Gauge Field Theories, Field Theories in Higher Dimensions, MTheory, Supersymmetric Gauge Theory

ArXiv EPrint: 1702.04058 


\section{Contents}

1 Introduction 1

2 S-duality of $6 \mathrm{~d}(2,0)$ theories on $\mathbb{R}^{4} \times T^{2} \quad 3$

$\begin{array}{lll}2.1 & \text { S-duality and its anomaly } & 10\end{array}$

$\begin{array}{ll}2.2 & \text { Derivation from M-strings } \\ 2.3\end{array}$

$\begin{array}{ll}2.36 \mathrm{~d}(2,0) \text { theories of } D_{N} \text { and } E_{N} \text { types } & 25\end{array}$

3 High temperature limit of the index $\quad 28$

3.1 Tests with $\mathrm{U}(1)$ partition function 34

3.26 d chiral anomalies on Omega-deformed $\mathbb{R}^{4} \times T^{2}$

4 Conclusions and remarks $\quad 42$

\section{Introduction}

Strong/weak-coupling duality, or S-duality, exists in a number of quantum systems. In $4 \mathrm{~d}$ gauge theories, it takes the form of electromagnetic duality, which inverts the gauge coupling and exchanges the roles of elementary charged particles and magnetic monopoles [1]. It is realized in the simplest manner in maximally supersymmetric Yang-Mills (SYM) theory [2]. In this case, the spectrum of dyons in the Coulomb phase was shown to exhibit $\operatorname{SL}(2, \mathbb{Z})$ duality [3], providing a robust evidence of S-duality. S-duality in quantum field theories has also been a cornerstone of developing string dualities [4]. In both QFT and string theory, S-duality provides valuable insights on the strongly coupled regions of the systems.

S-duality of maximal SYM has many implications. In particular, this duality is related to the existence of $6 \mathrm{~d}$ superconformal field theories called $(2,0)$ theories [5]. $4 \mathrm{~d}$ maximal SYM theories with gauge groups $\mathrm{U}(N), \mathrm{SO}(2 N), E_{N}$ are realized by compactifying $6 \mathrm{~d}(2,0)$ theories on small $T^{2}$. The $\mathrm{SL}(2, \mathbb{Z})$ duality originates from the modular transformation on $T^{2}$. On one side, this relation highlights the far-reaching implications of the $6 \mathrm{~d}$ CFTs to challenging lower dimensional systems. On the other hand, the 6d CFTs lack microscopic definitions, so that S-duality can provide useful clues to better understand the mysterious 6d CFTs. In this paper, we study the S-duality of the $6 \mathrm{~d}(2,0)$ theories compactified on finite $T^{2}$, and use it to explore some interesting properties of these systems.

Our key observable of the $6 \mathrm{~d}(2,0)$ theory is the partition function of the D0-D4 system. More precisely, we study the Witten index of the quantum mechanical $\mathrm{U}(k)$ gauge theory for $k$ D0-branes bound to $N$ separated D4-branes and fundamental open strings, and also study their generating function for arbitrary $k$. In M-theory, this system is made of $N$ M5-branes wrapping $S^{1}$. The D0-D4 systems describe the 6d $(2,0)$ CFT on M5-branes in 
the sectors with nonzero Kaluza-Klein momenta. From the viewpoint of 5d super-YangMills theory on D4-branes, this partition function is also known as Nekrasov's instanton partition function [6]. Although the 5d Yang-Mills description of its instanton solitons is UV incomplete, the D0-D4 system provides a UV complete description for computing the instanton partition function. The results in our paper rely only on the UV complete D0-D4 quantum mechanics.

The D0-D4 index was explored in [7]. From the 5d viewpoint, this is a partition function on $\mathbb{R}^{4} \times S^{1}$, where $S^{1}$ is the temporal circle for the Witten index. However, with D0-branes (Yang-Mills instantons) providing the Kaluza-Klein (KK) modes of M-theory, there are evidences that this index (multiplied by a $5 \mathrm{~d}$ perturbative part) is a partition function of the $6 \mathrm{~d}(2,0) \mathrm{SCFT}$ on $\mathbb{R}^{4} \times T^{2}$. See $[7,8]$ for the $A_{N-1}$ theories, and [9] for the $D_{N}$ theories.

Regarding the D0-D4 index as a 6d partition function on $\mathbb{R}^{4} \times T^{2}$, one can naturally ask if it transforms in a simple manner under the S-duality acting on $T^{2}$. In this paper, we establish the S-duality of the prepotential of this index, finding a simple anomaly of S-duality which we can naturally interpret with $6 \mathrm{~d}$ chiral anomalies. Note that the prepotential $F$ is the leading coefficient of the free energy $-\log Z \sim \frac{F(a, m)}{\epsilon_{1} \epsilon_{2}}$ when the socalled Omega deformation is taken to zero, $\epsilon_{1}, \epsilon_{2} \rightarrow 0$. $a$ and $m$ are Coulomb VEV and $5 \mathrm{~d} \mathcal{N}=1^{*}$ mass parameter, explained in section 2 . The anomaly of S-duality takes the following form. The prepotential $F$ can be divided into two parts, $F(a, m)=F_{\text {S-dual }}(a, m)+$ $F_{\text {anom }}(m)$, where $F_{\mathrm{S} \text {-dual }}$ is related to its $\mathrm{S}$-dual prepotential by a 6 d generalization of the Legendre transformation. (See section 2 for details.) $F_{\text {anom }}$ is a simple function which does not obey S-duality, thus named anomalous part. We find $F_{\text {anom }}$ in a closed form in section 2, which in particular is independent of the Coulomb VEV $a$.

This finding has two major implications. Firstly, similar result was found for the prepotential $F^{4 \mathrm{~d}}$ of the $4 \mathrm{~d} \mathcal{N}=2^{*}$ theory [10], related to our prepotential by taking the small $T^{2}$ limit. $F^{4 \mathrm{~d}}$ is also given by the sum $F_{\mathrm{S}-\text { dual }}^{4 \mathrm{~d}}(a, m)+F_{\text {anom }}^{4 \mathrm{~d}}(m)$, where $F_{\text {S-dual }}^{4 \mathrm{~d}}$ is self S-dual. Since $F_{\text {anom }}^{4 \mathrm{~d}}$ is independent of $a, F^{4 \mathrm{~d}}$ is S-dual in the Seiberg-Witten theory, which only sees $a$ derivatives of $F^{4 \mathrm{~d}}$. In our $6 \mathrm{~d}$ uplift, $F$ appearing in the Seiberg-Witten theory also does not see $F_{\text {anom }}(m)$ for the same reason.

Secondly, the partition function $Z$ itself is a Witten index of the $6 \mathrm{~d}$ theory on $\mathbb{R}^{4,1} \times S^{1}$. So the full prepotential $F=F_{\mathrm{S} \text {-dual }}+F_{\text {anom }}$ including the $a$ independent $F_{\text {anom }}$ is physically meaningful, as the leading part of the free energy $-\log Z$ when $\epsilon_{1}, \epsilon_{2}$ are small. At this stage, we note our key discovery that $F_{\text {anom }}$ contains a term proportional to $N^{3}$ in a suitabe large $N$ limit, to be explained in section 2. In particular, we further consider the limit in which the KK modes on the circle become light. This amounts to taking the chemical potential $\beta$ conjugate to the KK momentum (D0-brane charge) to be much smaller than the inverse-radius of $S^{1}$. The small $\beta$ limit is the strong coupling limit of the $5 \mathrm{~d}$ YangMills theory, or the limit in which the sixth circle decompactifies. This is the regime in which 6d CFT physics should be visible. The prepotential in this limit can be computed from our anomalous S-duality, since it relates the small $\beta$ (strong coupling) regime to the well-understood large $\beta$ (weak coupling) regime. $F_{\text {anom }}$ determines the small $\beta$ limit of the free energy, and makes it scale like $N^{3}$. We also show that the term in the asymptotic free 
energy proportional to $N^{3}$ is related to the chiral anomaly of the $6 \mathrm{~d}(2,0)$ theory, using the methods of [11]. These findings show that the number of $5 \mathrm{~d}$ KK fields for D0-brane bound states grows like $N^{3}$, as we decompactify the system to $6 \mathrm{~d}$.

The rest of this paper is organized as follows. In sections 2.1 and 2.2, we develop the anomalous S-duality of the prepotential and test it either by expanding $F$ in the $5 \mathrm{~d} \mathcal{N}=1^{*}$ mass $m$, or by making the 'M-string' expansion [8]. In section 2.3 , we discuss the $6 \mathrm{~d}(2,0)$ theories of $D_{N}$ and $E_{N}$ types. In section 3, we study the high temperature free energy and show that it scales like $N^{3}$ in a suitable large $N$ limit. In section 3.1, we test our result for $\mathrm{U}(1)$ theory. In section 3.2, we account for the imaginary part of the asymptotic free energy from $6 \mathrm{~d}$ chiral anomalies. Section 4 concludes with comments and future directions.

\section{S-duality of $6 \mathrm{~d}(2,0)$ theories on $\mathbb{R}^{4} \times T^{2}$}

We shall study the Witten index of the D0-D4 quantum mechanics, consisting of $k$ D0branes and $N$ D4-branes. This system is a quantum mechanical $\mathrm{U}(k)$ gauge theory with 8 supersymmetry and $\mathrm{U}(N)$ global symmetry. See, for instance, [7] for the details of this system. Here, we shall only explain some basic aspects. The bosonic variables consist of four Hermitian $k \times k$ matrices $a_{m} \sim a_{\alpha \dot{\beta}}$, two complex $k \times N$ matrices $q_{\dot{\alpha}}$, five Hermitian $k \times k$ matrices $\varphi^{I}$, and a quantum mechanical $\mathrm{U}(k)$ gauge field $A_{t}$. Here, $m=1,2,3,4$ is the vector index on $\mathbb{R}^{4}$ for the spatial worldvolume of the D4-branes. $\alpha$ and $\dot{\alpha}$ are doublet indices of $\mathrm{SU}(2)_{l}$ and $\mathrm{SU}(2)_{r}$ respectively, which form $\mathrm{SO}(4)$ rotation of $\mathbb{R}^{4}$. I $=1, \cdots, 5$ is the vector index on $\mathbb{R}^{5}$ transverse to the D4-branes. When $\varphi^{I}$ are all diagonal matrices, their eigenvalues are interpreted as D0-brane positions transverse to D4-branes. Similarly, when $a_{m}$ are all diagonal, their eigenvalues are interpreted as D0-brane positions along D4-brane worldvolume. $q_{\dot{\alpha}}$ represent internal degrees of freedom. The bosonic potential energy is given by

$$
V=\frac{1}{2} D^{i} D^{i}-\frac{1}{2}\left[\varphi^{I}, a_{m}\right]^{2}+\left|\varphi^{I} q_{\dot{\alpha}}\right|^{2}-\frac{1}{4}\left[\varphi^{I}, \varphi^{J}\right]^{2},
$$

where traces are assumed if necessary, $i=1,2,3$ is the $\mathrm{SU}(2)_{r}$ triplet index. $D^{i}$ are given by

$$
D^{i}=\left(\tau^{i}\right)_{\dot{\beta}}^{\dot{\alpha}}\left(q_{\dot{\alpha}} q^{\dagger \dot{\beta}}+\frac{1}{2}\left[a^{\dot{\beta} \alpha}, a_{\alpha \dot{\alpha}}\right]\right)
$$

where $\tau^{i}$ are the Pauli matrices.

This system flows in IR to two branches. Classically, they are described by two branches of moduli space satisfying $V=0$, or $D^{i}=0,\left[\varphi^{I}, a_{m}\right]=0, \varphi^{I} q_{\dot{\alpha}}=0$ and $\left[\varphi^{I}, \varphi^{J}\right]=0$. The first branch is obtained by taking $q_{\dot{\alpha}}=0$, and $a_{m}, \varphi^{I}$ to be diagonal matrices. The $k$ sets of eigenvalues of $\left(a_{m}, \varphi^{I}\right)$ represent the positions of $k$ D0-branes on $\mathbb{R}^{9}$, unbound to the D4-branes. The second branch is obtained by taking $\varphi^{I}=0$, and $q_{\dot{\alpha}}, a_{m}$ to satisfy $D^{i}=0$. After modding out by the $\mathrm{U}(k)$ gauge orbit, one can show that this branch is described by $4 N k$ real parameters. The two branches meet at $\varphi^{I}=0, q_{\dot{\alpha}}=0$. Far away from this intersection, each branch is described by a nonlinear sigma model (NLSM) on its moduli space. We are interested in the second branch, describing 6d CFT on M5-branes in 
the sector with $k$ units of KK momentum. The Witten index of the second branch can be computed easily by deforming the system by a Fayet-Iliopoulos (FI) parameter, shifting $D^{i}$ in (2.2) by three constant $\xi^{i}$. After this deformation, the first branch becomes non-BPS, since $q_{\dot{\alpha}}=0$ cannot solve $D^{i}=0$ with $\xi^{i} \neq 0$. So the Witten index acquires contributions only from the second branch.

One can understand the second branch from the low energy field theory of D4-branes, the 5d maximal SYM theory. D0-branes are realized in Yang-Mills theory as instanton solitons, classically described by finite energy stationary solutions of the following BPS equation,

$$
F_{m n}= \pm \frac{1}{2} \epsilon_{m n p q} F_{p q}, \quad m, n, p, q=1, \cdots, 4 .
$$

The finite energy solutions are labeled by the instanton number $k$, defined by

$$
k \equiv \frac{1}{16 \pi^{2}} \int_{\mathbb{R}^{4}} \operatorname{tr}(F \wedge F) \in \mathbb{Z} .
$$

We shall consider D0-branes rather than anti-D0-branes, with $k>0$, equivalently (2.3) with + sign. $k$ corresponds to the rank of the $\mathrm{U}(k)$ gauge group of the quantum mechanics. The solutions of $D^{i}=0$, modded out by the $\mathrm{U}(k)$ gauge orbit, provides the moduli space of self-dual instantons. The NLSM on the second branch can be obtained by the moduli space approximation of the instanton solitons. However, this moduli space is known to be singular, having the so-called small instanton singularities. Due to these singularities, the NLSM description is incomplete, and needs a UV completion. This is naturally interpreted as inheriting the UV incompleteness of the $5 \mathrm{~d}$ Yang-Mills description. Although we do not know how to UV complete the full 5d SYM, the NLSM can be UV completed to the U $(k)$ quantum mechanics.

We shall study the D0-D4 system in the Coulomb phase, with scalar vacuum expectation value $(\mathrm{VEV})$. For $\mathrm{U}(N)$ theories, the $\mathrm{VEV}$ is parametrized by constant $N \times N$ diagonal matrices $\Phi^{I}=\operatorname{diag}\left(\Phi_{1}^{I}, \cdots, \Phi_{N}^{I}\right)$. In the D0-D4 system, $\Phi^{I}$ deforms the bosonic potential $V$ as

$$
V=\frac{1}{2} D^{i} D^{i}-\frac{1}{2}\left[\varphi^{I}, a_{m}\right]^{2}-\frac{1}{4}\left[\varphi^{I}, \varphi^{J}\right]^{2}+\left|\varphi^{I} q_{\dot{\alpha}}-q_{\dot{\alpha}} \Phi^{I}\right|^{2} .
$$

The $N$ eigenvalues of $\Phi^{I}$ correspond to the positions of $N$ D4-branes on $\mathbb{R}^{5}$. We shall separate the D4-branes along a line, giving nonzero VEV to $\Phi^{5}$ only. In this setting, we shall study the BPS bound states of the D0-branes and the fundamental open strings stretched along the $\Phi^{5}$ direction, suspended between a pair of D4-branes. The bound states preserve 4 Hermitian supercharges. In $6 \mathrm{~d}(2,0)$ theory, we compactify a spatial direction on a circle with radius $R^{\prime}$. The BPS states saturate the bound $E \geq \frac{P}{R^{\prime}}+v_{i} q_{i}$, where $E$ is the energy, and $P$ is the quantized momentum on $S^{1}$ which is $k$ in the D0-D4 system. $v_{i}$ are the $N$ eigenvalues of the scalar $\Phi^{5}$, and $q_{i}$ 's are the $\mathrm{U}(1)^{N}$ electric charges in the Coulomb branch, satisfying $q_{1}+\cdots+q_{N}=0$. From the $6 \mathrm{~d}$ viewpoint, they are the self-dual strings with charges $q_{i}$ coming from open M2-branes, with $P$ units of momenta on them. We also define $H \equiv R^{\prime}\left(E-v_{i} q_{i}\right)$, which is the (dimensionless) energy on the self-dual strings.

The $6 \mathrm{~d}$ index is defined by

$$
Z\left(\tau, m, \epsilon_{1,2}, v\right)=\operatorname{Tr}\left[(-1)^{F} e^{2 \pi i \tau \frac{H+P}{2}} e^{-2 \pi i \bar{\tau} \frac{H-P}{2}} e^{\epsilon_{1}\left(J_{1}+J_{R}\right)+\epsilon_{2}\left(J_{2}+J_{R}\right)} e^{2 m J_{L}} e^{-v_{i} q_{i}}\right] .
$$


Here, $J_{1}, J_{2}$ are two Cartans rotating the two 2-planes of $\mathbb{R}^{4}, J_{L}, J_{R}$ are the Cartans of $\mathrm{SU}(2)_{L} \times \mathrm{SU}(2)_{R}=\mathrm{SO}(4) \subset \mathrm{SO}(5)$ unbroken by the VEV of $\Phi^{5}$. The measure is chosen so that it commutes with 2 of the 4 Hermitian supercharges preserved by the BPS states, or a complex supercharge $Q$ and its conjugate $Q^{\dagger}$. See [7] for the details. One also finds that $\frac{H-P}{2} \sim\left\{Q, Q^{\dagger}\right\}$. Since only the states saturating the BPS bound $H \geq P$ contribute to the index, $Z$ is independent of $\bar{\tau}$. With $H=P$ understood, the factor $e^{2 \pi i \tau \frac{H+P}{2}} \rightarrow e^{2 \pi i \tau P}$ weights the BPS states with the momentum $P$ along the circle. So $Z$ can be written as

$$
Z\left(\tau, m, \epsilon_{1,2}, v\right)=Z_{\mathrm{pert}}\left(m, \epsilon_{1,2}, v\right) \sum_{k=0}^{\infty} q^{k} Z_{k}\left(m, \epsilon_{1,2}, v\right)
$$

where $q \equiv e^{2 \pi i \tau}$, and $Z_{0} \equiv 1$ by definition. $Z$ can be computed in the weakly coupled type IIA regime, in which D0-branes are much heavier than the stretched fundamental strings. $Z_{k}$ is computed as the nonperturbative Witten index of the D0-D4 system with fixed $k$. $Z_{\text {pert }}$ comes from the zero modes at $P=0$, the perturbative open string modes on the D4-branes. This factor can also be understood as coming from the perturbative partition function of the 5d maximal SYM. Since we are in the weakly coupled regime, $Z_{\text {pert }}$ can be computed unambiguously from the quadratic part of the Yang-Mills theory. Although we compute $Z_{\text {pert }}$ and $Z_{k}$ in this special regime, we naturally expect the result to be valid at general type IIA coupling, since this is a Witten index independent of the continuous coupling.

$Z_{k}$ and $Z_{\text {pert }}$ are known for classical gauge groups. For $\mathrm{U}(N), Z_{k}$ is given by $[7,12-14]$

$$
Z_{k}=\sum_{Y_{i} ; \sum_{i=1}^{N}\left|Y_{i}\right|=k} \prod_{i, j=1}^{N} \prod_{s \in Y_{i}} \frac{\sinh \frac{E_{i j}(s)+m-\epsilon_{+}}{2} \sinh \frac{E_{i j}(s)-m-\epsilon_{+}}{2}}{\sinh \frac{E_{i j}(s)}{2} \sinh \frac{E_{i j}(s)-2 \epsilon_{+}}{2}}
$$

where $\epsilon_{ \pm} \equiv \frac{\epsilon_{1} \pm \epsilon_{2}}{2}$, and

$$
E_{i j}(s)=v_{i}-v_{j}-\epsilon_{1} h_{i}(s)+\epsilon_{2}\left(v_{j}(s)+1\right)
$$

The summation is made over $N$ Young diagrams $Y_{i}$ with total number of boxes $k$, and $s$ runs over all boxes of the Young diagram $Y_{i} . h_{i}(s)$ is the distance from $s$ to the right end of the Young diagram $Y_{i}$, and $v_{j}(s)$ is the distance from $s$ to the bottom end of the Young diagram $Y_{j}$. See [7] for the details. One often calls $Z_{\text {inst }} \equiv \sum_{k=0}^{\infty} q^{k} Z_{k}$ the instanton partition function.

$Z_{\text {pert }}$ is given by $[15,16]$

$$
Z_{\text {pert }}=\prod_{\alpha \in \text { adj }}\left[\frac{\tilde{\Gamma}_{3}\left(\frac{\alpha(v)+\epsilon_{+}+m}{2 \pi i} \mid \frac{\epsilon_{1}}{2 \pi i}, \frac{\epsilon_{2}}{2 \pi i}\right) \tilde{\Gamma}_{3}\left(\frac{\alpha(v)+\epsilon_{+}-m}{2 \pi i} \mid \frac{\epsilon_{1}}{2 \pi i}, \frac{\epsilon_{2}}{2 \pi i}\right)}{\tilde{\Gamma}_{3}\left(\frac{\alpha(v)}{2 \pi i} \mid \frac{\epsilon_{1}}{2 \pi i}, \frac{\epsilon_{2}}{2 \pi i}\right) \tilde{\Gamma}_{3}\left(\frac{\alpha(v)+2 \epsilon_{+}}{2 \pi i} \mid \frac{\epsilon_{1}}{2 \pi i}, \frac{\epsilon_{2}}{2 \pi i}\right)}\right]^{\frac{1}{2}}
$$

where $\tilde{\Gamma}_{3}\left(z \mid w_{1}, w_{2}\right) \equiv \Gamma_{3}\left(z \mid 1, w_{1}, w_{2}\right) \Gamma_{3}\left(1-z \mid 1,-w_{1},-w_{2}\right)$, and $\Gamma_{N}\left(z \mid w_{1}, \cdots, w_{N}\right)$ is the Barnes' Gamma function. As noted in [16], $\alpha$ in the adjoint representation includes Cartans, $\alpha=0$, for which ' $\Gamma_{3}\left(0 \mid \frac{\epsilon_{1}}{2 \pi i}, \frac{\epsilon_{2}}{2 \pi i}\right)$ ' in the denominator would diverge. For these $\alpha$, 
one replaces ' $\tilde{\Gamma}_{3}\left(0 \mid w_{1}, w_{2}\right)$ ' factors by $\tilde{\Gamma}_{3}^{\prime}\left(0 \mid w_{1}, w_{2}\right) \equiv \lim \left[z \tilde{\Gamma}_{3}\left(z \mid w_{1}, w_{2}\right)\right]$. See [16] for more details. For $t_{1} \equiv e^{\epsilon_{1}}<1, t_{2} \equiv e^{\epsilon_{2}}<1, Z_{\text {pert }}$ can be rewritten as

$$
Z_{\text {pert }}=e^{-\mathcal{F}} \prod_{\alpha \in \mathbf{a d j}} \prod_{n_{1}, n_{2} \geq 0}\left[\frac{\left(1-e^{\alpha(v)} t_{1}^{n_{1}} t_{2}^{n_{2}}\right)^{\prime}\left(1-e^{\alpha(v)} t_{1} t_{2} t_{1}^{n_{1}} t_{2}^{n_{2}}\right)}{\left(1-e^{\alpha(v)+\epsilon_{+} \pm m} t_{1}^{n_{1}} t_{2}^{n_{2}}\right)}\right]^{\frac{1}{2}}
$$

where prime here again means excluding the zero modes at $n_{1}=n_{2}=0$ for the Cartans $\alpha=0$. The overall factor $\mathcal{F}$ is given for gauge group $G$ by [15]

$$
\begin{aligned}
\mathcal{F}=-\frac{\pi i}{2} \sum_{\alpha \in \mathbf{a d j}}\left[\zeta_{3}\left(0, \frac{\alpha(v)}{2 \pi i} \mid 1, \frac{\epsilon_{1}}{2 \pi i}, \frac{\epsilon_{2}}{2 \pi i}\right)+\zeta_{3}\left(0, \frac{\alpha(v)+2 \epsilon_{+}}{2 \pi i} \mid 1, \frac{\epsilon_{1}}{2 \pi i}, \frac{\epsilon_{2}}{2 \pi i}\right)\right. \\
\left.-\zeta_{3}\left(0, \frac{\alpha(v)+\epsilon_{+} \pm m}{2 \pi i} \mid 1, \frac{\epsilon_{1}}{2 \pi i}, \frac{\epsilon_{2}}{2 \pi i}\right)\right] \\
=-\frac{\pi i}{2} \sum_{\alpha(v) \in \mathbf{a d j}} \frac{\epsilon_{+}^{2}-m^{2}}{2 \epsilon_{1} \epsilon_{2}}(1-2 \alpha(v))=\frac{\pi i\left(m^{2}-\epsilon_{+}^{2}\right)}{4 \epsilon_{1} \epsilon_{2}}|G|,
\end{aligned}
$$

where $\zeta_{3}$ is the Barnes' zeta function. When $t_{1}, t_{2}<e^{\alpha(v)}$ for all $\alpha \in \mathbf{a d j}, Z_{\text {pert }}$ is rewritten as

$$
Z_{\text {pert }}\left(v, \epsilon_{1,2}, m\right)=e^{-\mathcal{F}} P E\left[\frac{1}{2} \frac{\sinh \frac{m+\epsilon_{+}}{2} \sinh \frac{m-\epsilon_{+}}{2}}{\sinh \frac{\epsilon_{1}}{2} \sinh \frac{\epsilon_{2}}{2}} \chi_{\mathbf{a d j}}\left(e^{v}\right)+\frac{r}{2}\right],
$$

where $P E[f(x, y, z, \cdots)] \equiv \exp \left[\sum_{n=1}^{\infty} \frac{1}{n} f(n x, n y, n z, \cdots)\right], \chi_{\mathbf{a d j}} \equiv \sum_{\alpha \in \mathbf{a d j}} e^{\alpha(v)}$, and $r$ is the rank of gauge group which is $r=N$ for $\mathrm{U}(N)$. The term $\frac{r}{2}$ in $P E$ comes from excluding $r$ fermionic zero modes for the Cartans.

One may multiply an alternative perturbative factor $\check{Z}_{\text {pert }} \equiv e^{-\varepsilon_{0}}\left[Z_{\text {pert }}^{\mathrm{U}(1)}\right]^{N} \hat{Z}_{\text {pert }}$ to $Z_{\text {inst }}$, where $\left[Z_{\text {pert }}^{\mathrm{U}(1)}\right]^{N}$ is the perturbative partition function for the $N$ Cartans, $\hat{Z}_{\text {pert }}$ is defined by

$$
\hat{Z}_{\text {pert }}=P E\left[\frac{\sinh \frac{m+\epsilon_{+}}{2} \sinh \frac{m-\epsilon_{+}}{2}}{\sinh \frac{\epsilon_{1}}{2} \sinh \frac{\epsilon_{2}}{2}} \sum_{\alpha>0} e^{-\alpha(v)}\right],
$$

and

$$
\varepsilon_{0}=\frac{m^{2}-\epsilon_{+}^{2}}{2 \epsilon_{1} \epsilon_{2}} \sum_{\alpha>0}(\alpha(v)+\pi i) .
$$

Here all sums are over positive roots $\alpha>0$. This expression is well defined when all $\alpha(v)$ are positive for positive roots and larger than $m, \epsilon_{1,2}$. This expression will be useful when studying S-duality from the M-string viewpoint, in section 2.2. $Z_{\text {pert }}$ and $\check{Z}_{\text {pert }}$ are different in subtle ways, which shall not affect the studies of prepotential in this paper but has implications on the S-duality of $Z$, which we comment on in section 2.2. (2.14) has a more natural interpretation as the Witten index of charged W-bosons in the Coulomb phase [7]. However, as an abstract partition function, $Z_{\text {pert }}$ is more natural as it is manifestly Weyl-invariant. 
It will also be useful to know the simple structures of the Abelian partition function, $Z^{\mathrm{U}(1)}=Z_{\text {pert }}^{\mathrm{U}(1)} Z_{\text {inst }}^{\mathrm{U}(1)}$. Firstly, the perturbative $\mathrm{U}(1)$ partition function can be written as

$$
\begin{aligned}
Z_{\text {pert }}^{\mathrm{U}(1)} & =e^{-\frac{\pi i\left(m^{2}-\epsilon_{+}^{2}\right)}{4 \epsilon_{1} \epsilon_{2}}} P E\left[\frac{1}{2} \frac{\sinh \frac{m+\epsilon_{+}}{2} \sinh \frac{m-\epsilon_{+}}{2}}{\sinh \frac{\epsilon_{1}}{2} \sinh \frac{\epsilon_{2}}{2}}+\frac{1}{2}\right] \\
& =e^{-\frac{\pi i\left(m^{2}-\epsilon_{+}^{2}\right)}{4 \epsilon_{1} \epsilon_{2}}} P E\left[\frac{1}{2} \frac{\sinh \frac{m+\epsilon_{-}}{2} \sinh \frac{m-\epsilon_{-}}{2}}{\sinh \frac{\epsilon_{1}}{2} \sinh \frac{\epsilon_{2}}{2}}\right]
\end{aligned}
$$

at $e^{\epsilon_{1}}<1, e^{\epsilon_{2}}<1$, by following the discussions till (2.13) for $N=1$. The instanton part can be written as $[17]$

$$
Z_{\text {inst }}^{\mathrm{U}(1)}=\sum_{k=0}^{\infty} q^{k} Z_{k}=P E\left[\frac{\sinh \frac{m+\epsilon_{-}}{2} \sinh \frac{m-\epsilon_{-}}{2}}{\sinh \frac{\epsilon_{1}}{2} \sinh \frac{\epsilon_{2}}{2}} \frac{q}{1-q}\right],
$$

after summing over all Young diagrams in (2.8).

Given $Z=Z_{\text {pert }} Z_{\text {inst }}$, or $\check{Z}=\check{Z}_{\text {pert }} Z_{\text {inst }}$, one can write this partition function as

$$
Z=P E\left[\frac{f\left(\tau, m, \epsilon_{1,2}, v\right)}{2 \sinh \frac{\epsilon_{1}}{2} \cdot 2 \sinh \frac{\epsilon_{2}}{2}}\right] \equiv \exp \left[\sum_{n=1}^{\infty} \frac{1}{n} \frac{f\left(n \tau, n m, n \epsilon_{1,2}, n v\right)}{2 \sinh \frac{n \epsilon_{1}}{2} \cdot 2 \sinh \frac{n \epsilon_{2}}{2}}\right],
$$

or a similar expression for $\check{Z}$ using $\check{f}$. The expression appearing in $P E$ is called the single particle index, containing all the information on the BPS bound states. The coefficients of $f$ in fugacity expansion are also called Gopakumar-Vafa invariants [18, 19]. The factor $\frac{1}{2 \sinh \frac{\epsilon_{1}}{2} \cdot 2 \sinh \frac{\epsilon_{2}}{2}}$ comes from the center-of-mass zero modes of the particle on $\mathbb{R}^{4}$, which would have caused the path integral for $Z$ to diverge at $\epsilon_{1}=\epsilon_{2}=0$. So $\epsilon_{1,2}$ also plays the role of IR regulators. $f\left(\tau, m, \epsilon_{1,2}, v\right)$ takes into account the relative degrees of freedom of the bound state, in which $\epsilon_{1,2}$ are just chemical potentials. In particular, $\epsilon_{1,2} \rightarrow 0$ limit is smooth in $f$.

In this paper, we shall mostly discuss the limit $\epsilon_{1}, \epsilon_{2} \rightarrow 0$. In this limit, one finds

$$
Z_{\text {pert }} \sim \exp \left[-\frac{F_{\text {pert }}(v, m)}{\epsilon_{1} \epsilon_{2}}\right], \quad Z_{\text {inst }} \sim \exp \left[-\frac{F_{\text {inst }}(\tau, v, m)}{\epsilon_{1} \epsilon_{2}}\right]
$$

from (2.18). $F=F_{\text {pert }}+F_{\text {inst }}$ is the prepotential. $F_{\text {inst }}$ can be obtained from (2.8) after a straightforward but tedious calculation. $F_{\text {pert }}$ can be obtained from $(2.13)$, which is given by

$$
F_{\text {pert }}(v, m)=\frac{\pi i m^{2}}{4}|G|+\sum_{\alpha \in \mathbf{a d j}}\left(\operatorname{Li}_{3}\left(e^{-\alpha(v)}\right)-\frac{1}{2} \operatorname{Li}_{3}\left(e^{-(\alpha(v)+m)}\right)-\frac{1}{2} \operatorname{Li}_{3}\left(e^{-(\alpha(v)-m)}\right)\right),
$$

where $\operatorname{Li}_{s}(x)=\sum_{n=1}^{\infty} \frac{x^{n}}{n^{s}}$ for $|x|<1$, and can be continued to the complex $x$ plane with a branch cut. The first term coming from $\mathcal{F}$ will play no role in this paper. One way of obtaining (2.20) is to first take $v, m$ to be purely imaginary, to guarantee convergence of the sum in (2.13), and take the limit $\epsilon_{1,2} \rightarrow 0$ to obtain (2.20). Then, (2.20) can be 
analytically continued to complex $v, m$. One may alternatively start from $\check{Z}_{\text {pert }}$ and obtain its prepotential,

$$
\check{F}_{\text {pert }}=\frac{m^{2}}{2}\left(\pi i\left|\Delta_{+}\right|+\sum_{\alpha>0} \alpha(v)\right)+\sum_{\alpha>0}\left(2 \operatorname{Li}_{3}\left(e^{-\alpha(v)}\right)-\operatorname{Li}_{3}\left(e^{-\alpha(v) \pm m}\right)\right)+r F_{\text {pert }}^{\mathrm{U}(1)} .
$$

$\Delta_{+}$is the set of positive roots. Here, from the identity

$$
\operatorname{Li}_{n}\left(e^{2 \pi i x}\right)+(-1)^{n}\left(e^{-2 \pi i x}\right)=-\frac{(2 \pi i)^{n}}{n !} B_{n}(x)
$$

for $0<\operatorname{Re}(x) \leq 1$ and $\operatorname{Im}(x)<0$, where $B_{n}(x)$ 's are Bernoulli polynomials, one finds

$$
\mathrm{Li}_{3}\left(e^{x}\right)-\mathrm{Li}_{3}\left(e^{-x}\right)=-\frac{(2 \pi i)^{3}}{6} B_{3}\left(\frac{x}{2 \pi i}\right)
$$

for $\operatorname{Re}(x)>0$ and $0<\operatorname{Im}(x) \leq 2 \pi$. So for simplicity, let us assume $\operatorname{Re}(\alpha(v))> \pm \operatorname{Re}(m)$ for all positive roots $\alpha$, and also $\operatorname{Im}(\alpha(v))$ is chosen such that all $\operatorname{Im}(\alpha(v) \pm m)$ are within the range $(0,2 \pi]$ for positive roots. Then one finds

$$
\begin{aligned}
F_{\text {pert }}-\check{F}_{\text {pert }} & =-\frac{m^{2}}{2} \sum_{\alpha>0} \alpha(v)-\frac{(2 \pi i)^{3}}{6} \sum_{\alpha>0}\left[B_{3}\left(\frac{\alpha(v)}{2 \pi i}\right)-\frac{1}{2} B_{3}\left(\frac{\alpha(v) \pm m}{2 \pi i}\right)\right] \\
& =-\frac{\pi i m^{2}}{2}\left|\Delta_{+}\right|
\end{aligned}
$$

where we used $B_{3}(x)=x^{3}-\frac{3}{2} x^{2}+\frac{1}{2} x$. So at least in this setting, $F_{\text {pert }}$ and $\check{F}_{\text {pert }}$ differ only by a trivial constant independent of $v$. The last constant will play no role in this paper.

It will be helpful to consider the prepotential of the U(1) theory separately. From (2.16) and (2.17), the prepotential $f_{\mathrm{U}(1)}=F_{\text {pert }}^{\mathrm{U}(1)}+F_{\text {inst }}^{\mathrm{U}(1)}$ for the $\mathrm{U}(1)$ theory is given by

$$
f_{\mathrm{U}(1)}=\sum_{n=1}^{\infty}\left(2 \mathrm{Li}_{3}\left(q^{n}\right)-\mathrm{Li}_{3}\left(e^{m} q^{n}\right)-\mathrm{Li}_{3}\left(e^{-m} q^{n}\right)\right)+\frac{1}{2}\left(2 \mathrm{Li}_{3}(1)-\mathrm{Li}_{3}\left(e^{m}\right)-\mathrm{Li}_{3}\left(e^{-m}\right)\right)+\frac{\pi i m^{2}}{4}
$$

For studying the S-duality of this prepotential, it will be useful to make an expansion of $f_{\mathrm{U}(1)}$ in $m$. One first finds that the instanton part is given by

$$
\begin{aligned}
& \sum_{n=1}^{\infty}\left(2 \operatorname{Li}_{3}\left(q^{n}\right)-\mathrm{Li}_{3}\left(e^{m} q^{n}\right)-\mathrm{Li}_{3}\left(e^{-m} q^{n}\right)\right)=-m^{2} \sum_{n=1}^{\infty} \operatorname{Li}_{1}\left(q^{n}\right)-2 \sum_{j=1}^{\infty} \sum_{n=1}^{\infty} \frac{m^{2 j+2}}{(2 j+2) !} \operatorname{Li}_{1-2 j}\left(q^{n}\right) \\
& =m^{2} \sum_{n=1}^{\infty} \log \left(1-q^{n}\right)-2 \sum_{j, n, k=1}^{\infty} \frac{m^{2 j+2}}{(2 j+2) !} k^{2 j-1} q^{n k}=m^{2} \log \phi(\tau)-2 \sum_{j, k=1}^{\infty} \frac{m^{2 j+2}}{(2 j+2) !} \frac{k^{2 j-1} q^{k}}{1-q^{k}} \\
& =m^{2} \log \phi(\tau)+\sum_{j=1}^{\infty} \frac{m^{2 j+2}}{2 j(2 j+2) !}\left(E_{2 j}(\tau)-1\right),
\end{aligned}
$$

where $\phi(\tau)=\prod_{n=1}^{\infty}\left(1-q^{n}\right)=q^{-\frac{1}{24}} \eta(\tau)$ is the Euler function, and we used the identity

$$
\sum_{k=1}^{\infty} \frac{k^{2 j-1} q^{k}}{1-q^{k}}=-\frac{B_{2 j}}{4 j}\left(E_{2 j}(\tau)-1\right)
$$


for the Eisenstein series $E_{2 n}(\tau) . B_{n}$ are the Bernoulli numbers: $B_{1}= \pm \frac{1}{2}, B_{2 n+1}=0$,

$$
B_{0}=1, \quad B_{2}=\frac{1}{6}, \quad B_{4}=-\frac{1}{30}, \quad B_{6}=\frac{1}{42}, \quad B_{8}=-\frac{1}{30}
$$

and so on. The perturbative prepotential can be expanded in $m$ by using

$$
\operatorname{Li}_{n}\left(e^{z}\right)=\frac{z^{n-1}}{(n-1) !}\left(H_{n-1}-\log (-z)\right)+\sum_{k=0 ; k \neq n-1}^{\infty} \frac{\zeta(n-k)}{k !} z^{k}
$$

at small $z$, with $H_{n}=\sum_{p=1}^{n} \frac{1}{p}$. One finds

$$
\frac{1}{2}\left(2 \operatorname{Li}_{3}(1)-\operatorname{Li}_{3}\left(e^{m}\right)-\operatorname{Li}_{3}\left(e^{-m}\right)\right)=m^{2}\left(\frac{1}{2} \log m-\frac{3}{4}+\frac{1}{4} \log (-1)\right)+\sum_{j=1}^{\infty} \frac{B_{2 j} m^{2 j+2}}{2 j(2 j+2) !}
$$

Combining all, one obtains

$$
f_{\mathrm{U}(1)}=m^{2}\left(\frac{1}{2} \log m-\frac{3}{4}+\frac{\pi i}{2}+\log \phi(\tau)\right)+\sum_{n=1}^{\infty} \frac{m^{2 n+2} B_{2 n}}{2 n \cdot(2 n+2) !} E_{2 n}(\tau) .
$$

This will be useful later for understanding $N f_{\mathrm{U}(1)}$, as a part of the $\mathrm{U}(N)$ prepotential.

One can understand the chemical potentials from the viewpoint of the $4 \mathrm{~d}$ effective action in the Coulomb branch. The dimensionless variables $m, \epsilon_{1,2}, v$ take the form of

$$
m=R M, \quad \epsilon_{1,2}=R \varepsilon_{1,2}, \quad v=R a,
$$

where $R$ is the radius of the temporal circle of $\mathbb{R}^{4} \times S^{1} . M$ is the mass deformation parameter of the $4 \mathrm{~d} \mathcal{N}=2^{*}$ Yang-Mills theory, or the $5 \mathrm{~d} \mathcal{N}=1^{*}$ theory. (More precisely, $M$ is $2 \pi$ times the mass.) $\varepsilon_{1,2}$ are the Omega deformation parameters which have dimensions of mass. $a$ is the Coulomb VEV of the scalar field $\Phi^{5} . \tau$ is identified as

$$
\tau=i \frac{R}{R^{\prime}}
$$

where $R^{\prime}$ is the radius of the sixth circle. This is the inverse gauge coupling in $4 \mathrm{~d}$. $\tau$ can be complexified with a real part, given by the RR 1-form holonomy of type IIA theory on $S^{1}$.

The 4 dimensional limit of the partition function is obtained by taking $R \rightarrow 0$ with fixed $\tau, M, \varepsilon_{1,2}, a$. From (2.8), one finds that all sinh functions of $v, \epsilon_{1,2}, m$ are replaced by linear functions of $a, \varepsilon_{1,2}, M$, and the $R$ dependences cancel between numerator and denominator. As a result, the $4 \mathrm{~d}$ limit $Z_{k}^{4 \mathrm{~d}}$ of the instanton partition function is given by a rational function of $M, \varepsilon_{1,2}, a$ of degree 0 . This makes $Z_{\text {inst }}^{4 \mathrm{~d}}$ and $F_{\text {inst }}^{4 \mathrm{~d}}$ to enjoy a simple scaling property,

$$
Z_{\text {inst }}^{4 \mathrm{~d}}\left(\tau, \lambda M, \lambda \varepsilon_{1,2}, \lambda a\right)=Z_{\text {inst }}^{4 \mathrm{~d}}\left(\tau, M, \varepsilon_{1,2}, a\right), \quad F_{\text {inst }}^{4 \mathrm{~d}}(\tau, \lambda M, \lambda a)=\lambda^{2} F_{\text {inst }}^{4 \mathrm{~d}}(\tau, M, a) .
$$

This will be used in section 2.1 to provide two interpretations of the $4 \mathrm{~d}$ S-duality, and extend one version to $6 \mathrm{~d}$. As for the perturbative part $F_{\text {pert }}$, one can use (2.29) to obtain 
$F_{\text {pert }}^{4 \mathrm{~d}} \equiv \lim _{R \rightarrow 0} F_{\text {pert }}$. One finds

$$
\begin{aligned}
F_{\text {pert }}^{4 \mathrm{~d}}=\sum_{\alpha \in \text { adj }}[ & M^{2}\left(\log R-\frac{3}{4}\right)-\frac{\alpha(a)^{2}}{2} \log \alpha(a) \\
& \left.+\frac{(\alpha(a)+M)^{2}}{4} \log (\alpha(a)+M)+\frac{(\alpha(a)-M)^{2}}{4} \log (\alpha(a)-M)\right]
\end{aligned}
$$

where the first term independent of the Coulomb VEV is unphysical in the Seiberg-Witten theory. The perturbative prepotential satisfies the following pseudo-scaling property,

$$
F_{\text {pert }}^{4 \mathrm{~d}}(\lambda M, \lambda a)=\lambda^{2}\left(F_{\text {pert }}^{4 \mathrm{~d}}(M, a)+|G| \frac{M^{2}}{2} \log \lambda\right)
$$

which is homogenous and degree 2 up to a Coulomb VEV independent shift.

$Z_{\text {inst }}$ or $F_{\text {inst }}$ are only known as $q$ expansion when $q \ll 1$, or $\tau \rightarrow i \infty$. This is useful when the 'temperature' is much smaller than the Kaluz-Klein scale $\frac{1}{R^{\prime}}$, when the KK modes are 'heavy.' However, to study 6d SCFT, it is more interesting to explore the regime $q \rightarrow 1$, or $\tau \rightarrow i 0^{+}$, in which case the circle effectively decompactifies. The two regimes are weakly coupled and strongly coupled regimes, respectively. So if there is S-duality for the partition function on $\mathbb{R}^{4} \times T^{2}$, it will be helpful to study the interesting decompactifying regime from the well-understood region $\tau \rightarrow i \infty$. Developing the S-duality of the prepotential $F$ is the goal of this section. (In section 2.2, we also comment on the S-duality of the full partition function.)

\section{$2.1 \quad$ S-duality and its anomaly}

Following [10], we review the basic set up for studying the S-duality of 4 dimensional prepotential, and extend it to the $6 \mathrm{~d}$ theory on $T^{2}$.

The prepotential $F$ of general $4 \mathrm{~d} \mathcal{N}=2$ gauge theory determines the effective action in the Coulomb branch. The magnetic dual description uses the dual Coulomb VEV $a_{D}(a)$ and the dual prepotential $F_{D}\left(a_{D}\right)$, defined by the following Legendre transformation,

$$
a_{D}=\frac{1}{2 \pi i} \frac{\partial F}{\partial a}, \quad F_{D}\left(a_{D}\right)=\mathcal{L}[F](a) \equiv F(a)-2 \pi i a_{D} a=F-a \frac{\partial F}{\partial a} .
$$

For theories with higher rank $r>1, a$ has many components, $a_{i}$ with $i=1, \cdots, r$. Expressions like $a \frac{\partial}{\partial a}$ should be understood with contracted $i$ indices, i.e. $a \frac{\partial}{\partial a} \rightarrow \sum_{i=1}^{r} a_{i} \frac{\partial}{\partial a_{i}}$, whose sum structures will not be explicitly shown to make the notations simpler. For generic $\mathcal{N}=2$ theories, $F, F_{D}$ depend on other parameters like hypermultiplet masses and the coupling constant (or the dynamically generated scale $\Lambda$ instead of the coupling).

For $4 \mathrm{~d} \mathcal{N}=2^{*}$ theory, the prepotential $F^{4 \mathrm{~d}}$ (to be distinguished with the $6 \mathrm{~d}$ prepotential $F$ which we shall consider later) depends on the microscopic coupling constant $\tau$ and the adjoint hypermultiplet mass $M$. The prepotential can be divided into the classical, perturbative, and instanton contributions,

$$
F^{4 \mathrm{~d}}=F_{\mathrm{cl}}(\tau, a)+F_{\text {pert }}^{4 \mathrm{~d}}(a, M)+F_{\text {inst }}^{4 \mathrm{~d}}(\tau, a, M) \equiv F_{\mathrm{cl}}(\tau, a)+f^{4 \mathrm{~d}}(\tau, a, M)
$$


where $F_{\text {cl }}(\tau, a)=\pi i \tau a^{2}$, and $F_{\text {pert }}^{4 \mathrm{~d}} \cdot f^{4 \mathrm{~d}} \equiv F_{\text {pert }}^{4 \mathrm{~d}}+F_{\text {inst }}^{4 \mathrm{~d}}$ is the quantum prepotential. To study self S-dual theories, it is convenient to define $F_{D}^{4 \mathrm{~d}}$ as a function of the dual coupling $\tau_{D}=-\frac{1}{\tau}$. For the $4 \mathrm{~d} \mathcal{N}=2^{*}$ theory, $F_{D}^{4 \mathrm{~d}}$ is defined by

$$
F_{D}^{4 \mathrm{~d}}\left(\tau_{D}, a_{D}, M\right)=\mathcal{L}\left[F^{4 \mathrm{~d}}\right](\tau, a, M)=F^{4 \mathrm{~d}}(\tau, a, M)-a \frac{\partial F^{4 \mathrm{~d}}}{\partial a}(\tau, a, M) .
$$

Then, self S-duality exists if $F_{D}^{4 \mathrm{~d}}$ and $F^{4 \mathrm{~d}}$ are same function, $F_{D}^{4 \mathrm{~d}}(\tau, a, M)=F^{4 \mathrm{~d}}(\tau, a, M)$. This S-duality has been tested in detail in [10]. More precisely, it was found that

$$
F^{4 \mathrm{~d}}(\tau, a, M)=F_{\mathrm{S}-\mathrm{dual}}^{4 \mathrm{~d}}(\tau, a, M)+F_{\text {anom }}^{4 \mathrm{~d}}(\tau, M),
$$

where $F_{\mathrm{S}-\text { dual }}^{4 \mathrm{~d}}$ satisfies

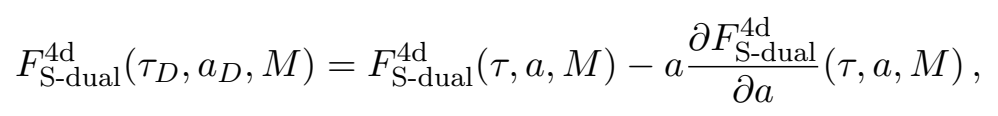

and $F_{\text {anom }}^{4 \mathrm{~d}}$ is an anomalous part of S-duality, depending on $\tau, M$ but is independent of the Coulomb VEV $a$ [10]. Since the Coulomb branch effective action is obtained by taking $a$ derivatives of $F^{4 \mathrm{~d}}, F^{4 \mathrm{~d}}$ and $F_{\mathrm{S} \text {-dual }}^{4 \mathrm{~d}}$ are identical in the Seiberg-Witten theory. This establishes the S-duality of the $4 \mathrm{~d} \mathcal{N}=2^{*}$ theory in the Coulomb branch effective action.

Let us rephrase the $4 \mathrm{~d}$ S-duality in a way that is suitable for $6 \mathrm{~d}$ extension. $F_{\text {inst }}^{4 \mathrm{~d}}$ satisfies the scaling property (2.34). Combining the perturbative part, one finds

$$
F^{4 \mathrm{~d}}(\tau, \lambda a, \lambda M)=\lambda^{2}\left(F^{4 \mathrm{~d}}(\tau, a, M)+|G| \frac{M^{2}}{2} \log \lambda\right) .
$$

Applying this to $F^{4 \mathrm{~d}}\left(\tau_{D}, a_{D}, m\right)$, one obtains

$$
F^{4 \mathrm{~d}}\left(\tau_{D}, a_{D} / \tau, M / \tau\right)=\tau^{-2} F^{4 \mathrm{~d}}\left(\tau_{D}, a_{D}, M\right)-\frac{M^{2}}{2 \tau^{2}} \log \tau .
$$

So the left hand side of (2.41) can be written as

$$
\begin{aligned}
F_{\text {S-dual }}^{4 \mathrm{~d}}\left(\tau_{D}, a_{D}, M\right)= & \tau^{2} F_{\mathrm{S} \text {-dual }}^{4 \mathrm{~d}}\left(\tau_{D}, a_{D} / \tau, M / \tau\right) \\
& +\frac{|G| M^{2}}{2} \log \tau+\tau^{2} F_{\text {anom }}^{4 \mathrm{~d}}\left(\tau_{D}, M / \tau\right)-F_{\text {anom }}^{4 \mathrm{~d}}\left(\tau_{D}, M\right) .
\end{aligned}
$$

Let us consider the structure of $F_{\text {anom }}^{4 \mathrm{~d}}$. Since the prepotential has mass dimension 2, one may think that its $M$ dependence is simply $M^{2}$. However, the perturbative part (2.35) shows that there is a term $\frac{r M^{2}}{2} \log M$ in $F^{4 d}$ which scales in an odd manner. In the computational framework of [10], which we shall explain below in our $6 \mathrm{~d}$ version, $F_{\mathrm{S}-\text { dual }}^{4 \mathrm{~d}}$ is by construction taken to be a series expansion in $M^{2}$. This means that the odd term $\frac{r M^{2}}{2} \log M$ should have been put in $F_{\text {anom }}^{4 \mathrm{~d}}$. Therefore, had one been doing the calculation of [10] using (2.35) as the perturbative part, one would have found that $F_{\text {anom }}^{4 \mathrm{~d}}=$ $\frac{r M^{2}}{2} \log M+M^{2}(\cdots)$, where $(\cdots)$ only depends on $\tau$. Using this structure, (2.44) can be rewritten as

$$
F_{\mathrm{S} \text {-dual }}^{4 \mathrm{~d}}\left(\tau_{D}, a_{D}, M\right)=\tau^{2} F_{\mathrm{S} \text {-dual }}^{4 \mathrm{~d}}\left(\tau_{D}, a_{D} / \tau, M / \tau\right)+(|G|-r) \frac{M^{2}}{2} \log \tau
$$


So defining

$$
\tilde{F}_{\text {S-dual }}^{4 \mathrm{~d}}(\tau, a, M)=F_{\mathrm{S}-\text { dual }}^{4 \mathrm{~d}}(\tau, a, M)-\frac{|G|-r}{2} M^{2} \log M,
$$

one finds that $\tilde{F}_{\text {S-dual }}^{4 d}$ satisfies

$$
\tau^{2} \tilde{F}_{\mathrm{S} \text {-dual }}^{4 \mathrm{~d}}\left(\tau_{D}, a_{D} / \tau, M / \tau\right)=\tilde{F}_{\mathrm{S} \text {-dual }}^{4 \mathrm{~d}}(\tau, a, M)-a \frac{\partial \tilde{F}_{\mathrm{S} \text {-dual }}^{4 \mathrm{~d}}}{\partial a}(\tau, a, M),
$$

instead of (2.41). To summarize, by trivially redefining $F_{\mathrm{S} \text {-dual }}^{4 \mathrm{~d}}$ and $F_{\text {anom }}^{4 \mathrm{~d}}$ by the last term of (2.46), one can reformulate the standard S-duality (2.41) as (2.47). Only (2.47) will naturally generalize to the S-duality on $\mathbb{R}^{4} \times T^{2}$.

Now we seek for the S-duality of the $6 \mathrm{~d}$ prepotential. Note that in $4 \mathrm{~d},(2.41)$ and (2.47) are equivalent by making a minor redefinition of $F_{\text {anom }}^{4 \mathrm{~d}}$, using $(2.42)$. In $6 \mathrm{~d}$, a property like (2.42) does not hold. Before making a quantitative study of the $6 \mathrm{~d}$ S-duality, we first explain that (2.47) is more natural in $6 \mathrm{~d}$. To discuss the $6 \mathrm{~d}$ prepotential, it is convenient to work with the dimensionless parameters $v, m, \epsilon_{1,2}$.

Firstly, in the $6 \mathrm{~d}$ theory compactified on $T^{2}$, the complex mass parameter $m$ is simply the holonomy of the background gauge field for $\mathrm{SU}(2)_{L}$ global symmetry, along the two sides of $T^{2}$. Then after making an S-duality of the torus, exchanging two sides of $T^{2}$, one naturally expects $m_{D}=\frac{m}{\tau}$. Let us briefly review this by taking a rectangular torus, for simplicity. In this case, the complex structure $\tau$ of the torus is purely imaginary. $\tau$ is related to the two radii of $T^{2}$ by

$$
\tau=i \frac{R}{R^{\prime}},
$$

where $R^{\prime}$ is the radius of the circle which compactifies the $6 \mathrm{~d}$ theory to $5 \mathrm{~d}$ SYM, and $R$ is the radius of another circle which compactifies the 5 d theory to $4 \mathrm{~d}$. The S-duality transformation exchanges $R \leftrightarrow R^{\prime}$. So the dual complex structure is $\tau_{D}=i \frac{R^{\prime}}{R}=-\frac{1}{\tau}$. More precisely, S-duality rotates the torus by 90 degrees on a plane. It also transforms the two $\mathrm{SU}(2)_{L}$ holonomies along the two circles. Let $\operatorname{Re}(M)$ be the holonomy on the circle with radius $R$, and $\operatorname{Im}(M)$ that on the circle with radius $R^{\prime}$. Under S-duality, one finds $\operatorname{Re}\left(M_{D}\right)=\operatorname{Im}(M), \operatorname{Im}\left(M_{D}\right)=-\operatorname{Re}(M)$. So one finds $M_{D}=-i M$. In $F, M$ appears in the dimensionless combination $m \equiv R M$, which transforms as

$$
m_{D}=R^{\prime} M_{D}=-i R^{\prime} M=-i \frac{R^{\prime}}{R} m=\frac{m}{\tau} .
$$

The final result holds for complex $\tau$. Similar property holds for $\epsilon_{1,2} \equiv R \varepsilon_{1,2}$, i.e. $\epsilon_{1,2}^{D}=\frac{\epsilon_{1,2}}{\tau}$. This makes the appearance of $\frac{M}{\tau}$ to be more natural on the left hand side of (2.47).

Secondly, let us discuss how $a$ should transform. In $4 \mathrm{~d}$, we already stated that

$$
a_{D}=\tau a+\frac{1}{2 \pi i} \frac{\partial f}{\partial a}
$$

naturally appears on the left hand side of (2.41). For simplicity, let us discuss these variables in the limit of large Coulomb VEV, $v \equiv R a \gg 1, a \gg m$. The second term can be ignored in this limit, yielding the semi-classical result $a_{D}=\tau a$. In this limit, we shall discuss what is the natural S-dual variable using the Abelian $6 \mathrm{~d}(2,0)$ theory. In $4 \mathrm{~d}, a_{D}=\tau a$ is a 
natural aspect of S-duality being electromagnetic duality. Also, it makes sense to multiply $a$ by a complex number $\tau$, since $a$ is a complex variable living on a plane. However, in 6d CFT on $T^{2}$, a lives on a cylinder. The real part of $a$ is the VEV of the real scalar in the $6 \mathrm{~d}$ self-dual tensor multiplet, which is noncompact. On the other hand, the imaginary part of a comes from the holonomy of the 2-form tensor field $B$ on $T^{2}$, implying that it is a periodic variable. So it does not good make sense to rotate $a$ living on a cylinder by complex $\tau$. More precisely, the $6 \mathrm{~d}$ scalar $\phi$ and the $5 \mathrm{~d}$ scalar $a$ are related by $a \sim R^{\prime} \phi$. So one finds

$$
a \sim R^{\prime}\left(\phi+i B_{12}\right)
$$

where 1 and 2 denote two directions of $T^{2}$. Thus, $v=R a \sim R R^{\prime}\left(\phi+i B_{12}\right)$ is invariant under $R \leftrightarrow R^{\prime}$, meaning that it makes more sense to set $v_{D} \approx v$ in the limit $v \gg 1$. Using the dimensionful variables, This requires one to use $\frac{a_{D}}{\tau} \approx a$ as the dual variable, instead of $a_{D} \approx \tau a$. This does not rotate the variable $a$ by a complex number, so makes better sense in $6 \mathrm{~d}$. Incidently, we have already found the alternative (but equivalent) statement (2.47) of S-duality which uses $\frac{a_{D}}{\tau}$ as the dual variable, instead of $a_{D}$. Note that the usage of $\frac{a_{D}}{\tau}=a+\frac{1}{2 \pi i \tau} \frac{\partial f^{4 \mathrm{~d}}}{\partial a}$ is valid even beyond the semi-classical limit $a \gg M$. Thus, in the $6 \mathrm{~d}$ uplift, it is natural and consistent to regard $v_{D} \equiv \frac{R a_{D}}{\tau}=v+\frac{1}{2 \pi i \tau} \frac{\partial f}{\partial v}$. as the dual variable. ${ }^{1}$

So it appears natural to seek for a $6 \mathrm{~d}$ generalization of (2.47) rather than (2.41). This is what we shall establish in the rest of this section. Namely, we shall find that the $6 \mathrm{~d}$ prepotential is divided into two,

$$
F=F_{\text {S-dual }}(\tau, v, m)+F_{\text {anom }}(\tau, m)
$$

where $v=R a, m=R M$, and $F_{\text {anom }}$ is independent of the Coulomb VEV. $F_{\text {S-dual }}$ satisfies

$$
\tau^{2} F_{\mathrm{S} \text {-dual }}\left(\tau_{D}=-\frac{1}{\tau}, v_{D}=v+\frac{1}{2 \pi i \tau} \frac{\partial f}{\partial v}, \frac{m}{\tau}\right)=F_{\mathrm{S}-\text { dual }}(\tau, v, m)-v \frac{\partial F_{\mathrm{S}-\text { dual }}}{\partial v}(\tau, v, m) .
$$

We have some freedom to choose $F_{\text {anom }}$, by adding/subtracting $v$ independent S-dual expressions to $F_{\text {anom }}, F_{\text {S-dual }}$. We shall explain that one can choose $F_{\text {anom }}$ as

$$
F_{\text {anom }}=N f_{\mathrm{U}(1)}(\tau, m)+\frac{N^{3}-N}{288} m^{4} E_{2}(\tau)
$$

where $f_{\mathrm{U}(1)}$ is the $\mathrm{U}(1)$ prepotential (2.25). The first term $N f_{\mathrm{U}(1)}$ comes from the $N 6 \mathrm{~d}$ Abelian tensor multiplets in $\mathrm{U}(1)^{N}$, which has their own S-duality anomaly. The second term of $F_{\text {anom }}$ is one of the key findings of this paper, which comes from the charged part of the partition function. After replacing $m=M R$, and multiplying $\frac{1}{R^{2}}$ to the above $F_{\text {anom }}$ to get to the conventionally normalized prepotential (as noted in footnote 1), one can take the $4 \mathrm{~d}$ limit of $F_{\text {anom. }}$. The second term proportional to $N^{3}-N$ vanishes in the $4 \mathrm{~d}$ limit $R \rightarrow 0$, as it is proportional to $M^{4} R^{2}$.

\footnotetext{
${ }^{1}$ Here, one may wonder that $f$ appearing on the right hand side should have been $R^{2} f$. However, we shall define the prepotential as the coefficient of the dimensionless $\frac{1}{\epsilon_{1} \epsilon_{2}},-\log Z \sim \frac{f}{\epsilon_{1} \epsilon_{2}}$, rather than $\frac{f^{4 \mathrm{~d}}}{\varepsilon_{1} \varepsilon_{2}}$ that is conventional in the Seiberg-Witten theory, making $f$ dimensionless. Namely, $f_{\text {ours }}$ in $6 \mathrm{~d}$ is related to the conventionally normalized prepotential by $f_{\text {ours }}=R^{2} f_{\text {conventional }}$.
} 
With the motivations and results given, we now properly set up the calculation and show the claims made above. As in $4 \mathrm{~d}$, we decompose the $6 \mathrm{~d}$ prepotential as

$$
F(\tau, v, m)=F_{\mathrm{cl}}+F_{\text {pert }}+F_{\text {inst }} \equiv F_{\mathrm{cl}}+f,
$$

where $F_{\mathrm{cl}} \equiv \pi i \tau v^{2}$. The prepotential is S-dual if it satisfies

$$
\tau^{2} F\left(\tau_{D}=-\frac{1}{\tau}, v_{D}=v+\frac{1}{2 \pi i \tau} \frac{\partial f}{\partial v}, m_{D}=\frac{m}{\tau}\right)=F(\tau, v, m)-v \frac{\partial F}{\partial v}(\tau, v, m) .
$$

We first study the structures of this equation, before showing that it is satisfied by our $F_{\mathrm{S} \text {-dual }}$. Firstly, replacing $F$ by $F_{\mathrm{cl}}$, one can check that S-duality trivially holds at the classical level:

$$
\tau^{2} F_{\mathrm{cl}}\left(\tau_{D}, v_{D}\right)=\tau^{2}\left[-\frac{\pi i}{\tau} v^{2}\right]=-\pi i \tau v^{2}=F_{\mathrm{cl}}(\tau, v)-v \frac{\partial F_{\mathrm{cl}}}{\partial v}(\tau, v)
$$

where $v_{D}$ is replaced by its classical value $v_{D}=v$ (formally at $f=0$ ). Now we subtract (2.56) by (2.57) to find the following condition for the quantum prepotential $f$ :

$$
\tau^{2} f\left(-\frac{1}{\tau}, v+\frac{1}{2 \pi i \tau} \frac{\partial f}{\partial v}, \frac{m}{\tau}\right)=f(\tau, v, m)+\frac{1}{4 \pi i \tau}\left(\frac{\partial f}{\partial v}(\tau, v, m)\right)^{2} .
$$

We are going to study the last equation. Note again that the effective action in the Coulomb branch only contains $v$ derivatives of $F$, or $f$. Thus, in Seiberg-Witten theory, $f$ is ambiguous by addition of $v$ independent functions, possibly depending on $\tau$ and $m$. However, the S-duality requirement (2.58) is sensitive to the value of $f$, including the $v$ independent part. So when one tries to establish the S-duality of the Coulomb branch effective action, one should have in mind that one may have to add suitable Coulomb VEV independent terms to $f$ computed microscopically from $Z$.

Following [10], we shall establish the S-duality (2.58) and its anomaly (2.54) by expanding $f$ in the mass $m$ when it is small enough. We shall still get an exact statement (2.54), which we check for certain orders in $m$. One should however have in mind that the exact statement (2.54) may be valid only within a finite region of $m, v$ in the complex planes. In section 2.2, we shed more lights on the exactness of (2.54), by making an M-string expansion [8].

As studied in the $4 \mathrm{~d}$ limit [10], there is a natural way of achieving the S-duality requirement (2.58). This is to require that $f$ is expanded in quasi-modular forms of suitable weights. To precisely explain its meaning, we first expand $f$ in $m$ as

$$
f(\tau, v, m)=\sum_{n=1}^{\infty} m^{2 n} f_{n}(\tau, v)
$$

This series makes sense as follows. Firstly, the $m \rightarrow 0$ limit exhibits enhanced maximal supersymmetry. So at $m=0$, the classical prepotential $F_{\mathrm{cl}}=\pi i \tau v^{2}$ acquires no quantum corrections, meaning that $f$ vanishes at $m=0$. Also, the prepotential is an even function 
of $m$, which restrict the expansion as above. ${ }^{2}$ Then, following [10], we require that $f_{n}$ is a quasi-modular form of weight $2 n-2$, which means the following. Quasi-modular forms are polynomials of the first three Eisenstein series $E_{2}, E_{4}, E_{6}$, where each series has weight 2, 4, 6 respectively under S-duality in the following sense:

$$
E_{2}(-1 / \tau)=\tau^{2}\left(E_{2}+\frac{6}{\pi i \tau}\right), \quad E_{4}(-1 / \tau)=\tau^{4} E_{4}(\tau), \quad E_{6}(-1 / \tau)=\tau^{6} E_{6}(\tau) .
$$

More concretely, they are given by

$$
E_{2}=1-24 \sum_{n=1}^{\infty} \frac{n q^{n}}{1-q^{n}}, \quad E_{4}(\tau)=1+240 \sum_{n=1}^{\infty} \frac{n^{3} q^{n}}{1-q^{n}}, \quad E_{6}=1-504 \sum_{n=1}^{\infty} \frac{n^{5} q^{n}}{1-q^{n}} .
$$

Higher Eisenstein series $E_{2 n}$ are polynomials of $E_{4}, E_{6}$ with weight $2 n$. To study the quasimodular property, it is helpful to decompose their dependence on $\tau$ into the dependence through $E_{2}$ and the dependence through $E_{4}, E_{6}$. We thus write $f_{n}\left(\tau, v, E_{2}(\tau)\right)$, where the $\tau$ dependence through $E_{2}$ is explicitly shown. A weight $2 n-2$ quasi-modular form $f_{n}$ satisfies

$$
f_{n}\left(-1 / \tau, v, E_{2}(-1 / \tau)\right)=\tau^{2 n-2} f_{n}\left(\tau, v, E_{2}(\tau)+\delta\right),
$$

where $\delta \equiv \frac{6}{\pi i \tau}$. In terms of $f$, this is equivalent to

$$
\tau^{2} f\left(-\frac{1}{\tau}, v, \frac{m}{\tau}, E_{2}(-1 / \tau)\right)=f\left(\tau, v, m, E_{2}(\tau)+\delta\right) .
$$

We now investigate how quasi-modularity is related to the S-duality (2.58). One can make (2.63) to be equivalent to (2.58) by specifying the $E_{2}$ dependence of $f$, which we now turn to.

Let us first try to find the desired $E_{2}$ dependence, by requiring both (2.58) and (2.63). By applying (2.63) to $f\left(-\frac{1}{\tau}, v_{D}, \frac{m}{\tau}, E_{2}(-1 / \tau)\right)$, one obtains

$$
\tau^{2} f\left(-\frac{1}{\tau}, v+\frac{\delta}{12} \frac{\partial f}{\partial v}, \frac{m}{\tau}, E_{2}(-1 / \tau)\right)=f\left(\tau, v+\frac{\delta}{12} \frac{\partial f}{\partial v}, m, E_{2}(\tau)+\delta\right),
$$

where again recall that $\delta \equiv \frac{6}{\pi i \tau}$. Combining this with (2.58), one obtains

$$
f\left(\tau, v+\frac{\delta}{12} \frac{\partial f}{\partial v}, m, E_{2}(\tau)+\delta\right)=f\left(\tau, v, m, E_{2}(\tau)\right)+\frac{\delta}{24}\left(\frac{\partial f}{\partial v}\left(\tau, v, m, E_{2}(\tau)\right)\right)^{2} .
$$

We want to make this equation to hold, by specifying a particular $E_{2}$ dependence of $f$. [10] showed that the desired $E_{2}$ dependence is

$$
\frac{\partial f}{\partial E_{2}}=-\frac{1}{24}\left(\frac{\partial f}{\partial v}\right)^{2}
$$

For the sake of completeness, we repeat the logics presented in [10] and expand it to make a proof. In fact, we shall make a stronger claim than needed. Namely, we need to find

\footnotetext{
${ }^{2}$ Strictly speaking, there is a term $\frac{r m^{2}}{2} \log m$ in the perturbative part, which is easiest to see from the $4 \mathrm{~d}$ limit (2.35). However, we shall expand $f_{\mathrm{S}-\mathrm{dual}}$ as (2.59), while the term $\frac{r m^{2}}{2} \log m$ is moved to $F_{\text {anom }}$.
} 
the $E_{2}$ dependence of $f$ which guarantees (2.65) only at $\delta=\frac{6}{\pi i \tau}$. However, we shall show that (2.66) guarantees (2.65) for arbitrary independent parameter $\delta$, and then set $\delta=\frac{6}{\pi i \tau}$ later.

As a warm-up, we follow [10] to make a series expansion of the left hand side of (2.65) in small $\delta$, to see how (2.66) guarantees (2.65) at low orders. One finds that

$$
\text { (l.h.s.) }=f+\frac{\delta}{12}\left(\frac{\partial f}{\partial v}\right)^{2}+\delta \frac{\partial f}{\partial E_{2}}+\mathcal{O}\left(\delta^{2}\right) .
$$

So at $\delta^{0}$ and $\delta^{1}$ orders, one finds that it agrees with the right hand side if (2.66) is met.

Now assuming (2.66), we consider whether (2.65) is satisfied in full generality. To this end, we take $\delta$ derivative of both sides of (2.65), at fixed $\tau, v, E_{2}$,

$$
\left.\frac{\partial}{\partial \delta}(\text { l.h.s. })=\frac{1}{12} \frac{\partial f}{\partial v} \frac{\partial \tilde{f}}{\partial \tilde{v}}+\frac{\partial \tilde{f}}{\partial \tilde{E}_{2}}=\frac{1}{12} \frac{\partial f}{\partial v} \frac{\partial \tilde{f}}{\partial \tilde{v}}-\frac{1}{24}\left(\frac{\partial \tilde{f}}{\partial \tilde{v}}\right)^{2}, \quad \frac{\partial}{\partial \delta} \text { (r.h.s. }\right)=\frac{1}{24}\left(\frac{\partial f}{\partial v}\right)^{2}
$$

where for simplicity, we defined

$$
\tilde{v}=v+\frac{\delta}{12} \frac{\partial f}{\partial v}, \quad \tilde{E}_{2}=E_{2}+\delta, \quad \tilde{f}=f\left(\tau, \tilde{v}, \tilde{E}_{2}\right) .
$$

Note that at the second step of the first equation in (2.68), we used $\frac{\partial \tilde{f}}{\partial \tilde{E}_{2}}=-\frac{1}{24}\left(\frac{\partial \tilde{f}}{\partial \tilde{v}}\right)^{2}$ which follows from (2.66). If (2.65) holds for general $\delta$, its first derivative would yield

$$
\frac{\partial \tilde{f}}{\partial \tilde{v}}=\frac{\partial f}{\partial v},
$$

which one can show by using (2.68). On the other hand, (2.70) together with the $\mathcal{O}\left(\delta^{0}\right)$ component of (2.65) is equivalent to (2.65), since the $\mathcal{O}\left(\delta^{0}\right)$ component is the only information lost by taking $\delta$ derivative. However, we have already shown around (2.67) that the $\mathcal{O}\left(\delta^{0}\right)$ component of (2.65) is satisfied. Therefore, showing (2.70) will be equivalent to showing (2.65). So will show (2.70) by assuming (2.66). We take $\delta$ derivative of $\frac{\partial \tilde{f}}{\partial \tilde{v}}-\frac{\partial f}{\partial v}$ at fixed $v, E_{2}$. Again using (2.66), one obtains

$$
\frac{\partial}{\partial \delta}\left(\frac{\partial \tilde{f}}{\partial \tilde{v}}-\frac{\partial f}{\partial v}\right)=-\frac{1}{12} \frac{\partial^{2} \tilde{f}}{\partial \tilde{v}^{2}}\left(\frac{\partial \tilde{f}}{\partial \tilde{v}}-\frac{\partial f}{\partial v}\right)
$$

So if $\frac{\partial \tilde{f}}{\partial \tilde{v}}-\frac{\partial f}{\partial v}$ is zero at a particular value of $\delta,(2.71)$ guarantees that it is zero at different values of $\delta$. Since we already checked around (2.67) that (2.65) is true up to $\mathcal{O}\left(\delta^{1}\right)$, we have shown that $(2.70)$ holds at $\mathcal{O}\left(\delta^{0}\right)$, or that $\frac{\partial \tilde{f}}{\partial \tilde{v}}-\frac{\partial f}{\partial v}=0$ at $\delta=0$. This establishes that (2.66) implies (2.70), and in turn that (2.66) implies (2.65). Finally, we insert $\delta=\frac{6}{\pi i \tau}$.

To summarize till here, (2.65) holds if $f$ satisfies (2.66). But (2.65) and (2.63) implies the S-duality relation (2.58). Therefore, S-duality requirement (2.58) is satisfied if $f$ satisfies the quasi-modular property (2.63) and the modular anomaly equation (2.66). In the rest of this subsection, we shall discuss the last two equations.

Following and extending [10], we show that the prepotential $f$ obeys the two properties (2.63), (2.66), up to an anomalous part which is independent of the Coulomb VEV $v$. 
Again following [10], our strategy is to first find a prepotential $f_{\mathrm{S} \text {-dual }}$ in a series of $m^{2}$ which satisfies both (2.63) and (2.66). Then we show that $f-f_{\text {S-dual }}$ is independent of $v$.

We expand $f_{\mathrm{S} \text {-dual }}$ like $(2.59), f_{\mathrm{S} \text {-dual }}=\sum_{n=1}^{\infty} m^{2 n} f_{n}(\tau, v)$. (2.66) is given in terms of $f_{n}$ by

$$
\frac{\partial f_{n}}{\partial E_{2}}=-\frac{1}{24} \sum_{m=1}^{n-1} \frac{\partial f_{m}}{\partial v} \frac{\partial f_{n-m}}{\partial v}
$$

for $n \geq 2$. This equation can be used to recursively compute $f_{n}$. Namely, once we know $f_{m}$ for $m=1, \cdots, n-1$, one can integrate the right hand side of (2.72) with $E_{2}$ to get $f_{n}$, up to an integration constant independent of $E_{2}$. The integration constant is a polynomial of $E_{4}$ and $E_{6}$ with modular weight $2 n-2$, whose coefficients depend only on $v$. These integration constants depending on $v$ can be fixed once we know a few low order coefficients of $f$ in $q$ expansion. Also, to start the recursive construction, the first coefficient $f_{1}$ at $m^{2}$ should be known. It will turn out that this can be also fixed by the known perturbative part $f_{\text {pert }}$ [10]. This way, one can recursively generate the coefficients of $f_{\text {S-dual }}$ from (2.63), (2.66) and the knowledge of the few low order coefficients of $f$ in $q$ expansion. We emphasize here that our purpose of making a recursive construction of $f_{\text {S-dual }}$ is to show that the Coulomb VEV dependent part of $f$ is S-dual. Therefore, while fixing the integration constants and $f_{1}$ in $f_{\mathrm{S} \text {-dual }}$ by using the low order $q$ expansion coefficients of $f$, it suffices to use $f$ up to the addition of any convenient expression independent of $v$. So for technical reasons, we shall fit these integration constants and $f_{1}$ by comparing $f_{\text {S-dual }}$ with

$$
f(\tau, v, m)-N f_{\mathrm{U}(1)}(\tau, m)
$$

rather than $f$ itself. Note that $N f_{\mathrm{U}(1)}$ is the prepotential contribution from $\mathrm{U}(1)^{N}$ Cartan part, coming from D0-branes bound to D4-branes but unbound to W-bosons which see $v$. One reason for comparing with $f-N f_{\mathrm{U}(1)}$ is that $f_{\mathrm{U}(1)}$ does not admit a power series expansion in $m^{2}$ like (2.59). The S-duality anomaly of $N f_{\mathrm{U}(1)}$ can be calculated separately from (2.31).

With these understood, we start the recursive contruction by determining $f_{1}$. This can be fixed solely from the perturbative part of $\left(f-N f_{\mathrm{U}(1)}\right)_{\text {pert }}$ [10]. Namely, when instantons are bound to $\mathrm{W}$-bosons, there are fermion zero modes which provide at least a factor of $m^{4}$ in $f$. This means that $m^{2}$ term $f_{1}$ should come from the perturbative part only. This fact can also be straightforwardly checked from the microscopic calculus. So one finds

$$
f_{1}=\left.\left(f-N f_{\mathrm{U}(1)}\right)_{\mathrm{pert}}\right|_{m^{2}}=-\frac{1}{2} \sum_{\alpha \in \Delta} \operatorname{Li}_{1}\left(e^{\alpha(v)}\right)=\frac{1}{2} \sum_{\alpha \in \Delta} \log \left(1-e^{\alpha(v)}\right),
$$

where $\Delta$ is the set of roots of $\mathrm{U}(N)$.

One can then compute $f_{2}$ using $(2.72)$ at $n=2$,

$$
\frac{\partial f_{2}}{\partial E_{2}}=-\frac{1}{24}\left(\frac{\partial f_{1}}{\partial v}\right)^{2}=-\frac{1}{96} \sum_{\alpha, \beta \in \Delta} \frac{\alpha \cdot \beta}{\left(1-e^{\alpha(v)}\right)\left(1-e^{\beta(v)}\right)} .
$$

One can integrate it with $E_{2}$, to obtain

$$
f_{2}=-\frac{E_{2}(\tau)}{96} \sum_{\alpha, \beta \in \Delta} \frac{\alpha \cdot \beta}{\left(1-e^{\alpha(v)}\right)\left(1-e^{\beta(v)}\right)} .
$$


There is no integration constant at weight 2 . To proceed, we study the properties of the $\mathrm{U}(N)$ roots. $\Delta$ consists of vectors of the form $e_{i}-e_{j}, i \neq j, i, j=1, \cdots, N$, where $e_{i}$ are $N$ orthogonal unit vectors. $\alpha \cdot \beta$ takes following possible values,

$$
\begin{aligned}
& \alpha \cdot \beta= \pm 2 \text { if } \pm \beta=\alpha \\
& \alpha \cdot \beta= \pm 1 \text { if } \pm \beta \in \Psi(\alpha) \\
& \alpha \cdot \beta=0 \quad \text { otherwise, }
\end{aligned}
$$

where $\Psi(\alpha)$ is given for $\alpha=e_{i}-e_{j}$ by

$$
\Psi\left(e_{i}-e_{j}\right)=\left\{k \neq i, j: e_{i}-e_{k}, e_{k}-e_{j}\right\} .
$$

For a given $\alpha$, there are $2(N-2)$ elements of $\Psi(\alpha)$. Using this, one finds

$$
\begin{aligned}
f_{2} & =-\frac{E_{2}(\tau)}{96}\left[\sum_{\alpha \in \Delta} \frac{2}{1-e^{\alpha(v)}}\left(\frac{1}{1-e^{\alpha(v)}}-\frac{1}{1-e^{-\alpha(v)}}\right)+\sum_{\alpha \in \Delta} \sum_{\beta \in \Psi(\alpha)} \frac{1}{1-e^{\alpha(v)}}\left(\frac{1}{1-e^{\beta(v)}}-\frac{1}{1-e^{-\beta(v)}}\right)\right] \\
& =-\frac{E_{2}(\tau)}{96}\left[\sum_{\alpha \in \Delta} \frac{4}{\left(1-e^{\alpha(v)}\right)^{2}}-\sum_{\alpha \in \Delta} \frac{2(N-1)}{1-e^{\alpha(v)}}+\sum_{\alpha \in \Delta} \sum_{\beta \in \Psi(\alpha)} \frac{2}{\left(1-e^{\alpha(v)}\right)\left(1-e^{\beta(v)}\right)}\right]
\end{aligned}
$$

where we used $\frac{1}{1-e^{\alpha(v)}}-\frac{1}{1-e^{-\alpha(v)}}=\frac{2}{1-e^{\alpha(v)}}-1$. Using $\frac{1}{\left(1-e^{\alpha(v)}\right)^{2}}=\frac{1}{1-e^{\alpha(v)}}+\frac{e^{\alpha(v)}}{\left(1-e^{\alpha(v))^{2}}\right.}=$ $\frac{1}{1-e^{\alpha(v)}}+\operatorname{Li}_{-1}\left(e^{-\alpha(v)}\right)$, the first term can be rewritten so that

$$
f_{2}=-\frac{E_{2}(\tau)}{96}\left[4 \sum_{\alpha \in \Delta} \operatorname{Li}_{-1}\left(e^{\alpha(v)}\right)-\sum_{\alpha \in \Delta} \frac{2(N-3)}{1-e^{\alpha(v)}}+\sum_{\alpha \in \Delta} \sum_{\beta \in \Psi(\alpha)} \frac{2}{\left(1-e^{\alpha(v)}\right)\left(1-e^{\beta(v)}\right)}\right] .
$$

Here, one can simplify the second term by using

$$
\sum_{\alpha \in \Delta} \frac{1}{1-e^{\alpha(v)}}=\frac{1}{2} \sum_{\alpha \in \Delta}\left(\frac{1}{1-e^{\alpha(v)}}+\frac{1}{1-e^{-\alpha(v)}}\right)=\frac{1}{2} \sum_{\alpha \in \Delta} 1=\frac{N(N-1)}{2} .
$$

Also, using $\frac{1}{\left(1-e^{x}\right)\left(1-e^{y}\right)}+\frac{1}{\left(1-e^{-x}\right)\left(1-e^{y-x}\right)}+\frac{1}{\left(1-e^{x-y}\right)\left(1-e^{-y}\right)}=1$, one finds

$$
\begin{aligned}
& \sum_{\alpha \in \Delta} \sum_{\beta \in \Psi(\alpha)} \frac{1}{\left(1-e^{\alpha(v)}\right)\left(1-e^{\beta(v)}\right)}=\sum_{i \neq j} \sum_{k \neq i, j}\left[\frac{1}{\left(1-e^{v_{i}-v_{j}}\right)\left(1-e^{v_{i}-v_{k}}\right)}+\frac{1}{\left(1-e^{v_{i}-v_{j}}\right)\left(1-e^{v_{k}-v_{j}}\right)}\right] \\
& =\frac{1}{3} \sum_{i \neq j \neq k \neq i}[(i, j, k)+(j, k, i)+(k, i, j)]=\sum_{i \neq j \neq k \neq i} \frac{2}{3}=\frac{2}{3} N(N-1)(N-2),
\end{aligned}
$$

where at the second step we symmetrized the summand by making a cyclic permutation of $i, j, k$. This simplifies the third term. One thus finds

$$
f_{2}=-\frac{E_{2}(\tau)}{24}\left[\sum_{\alpha \in \Delta} \operatorname{Li}_{-1}\left(e^{\alpha(v)}\right)+\frac{N^{3}-N}{12}\right]
$$

at $\mathcal{O}\left(m^{4}\right)$ order. 
Before proceeding to higher order coefficients $f_{n}$ with $n \geq 3$, let us first discuss $f_{2}$ that we computed by requiring S-duality of $f_{\text {S-dual }}$. Note that at $m^{4}$, we have obtained an all order result in the instanton expansion, coming from $E_{2}(\tau)=1-24 q-72 q^{2}-96 q^{3}-$ $168 q^{4} \cdots$. So from the microscopic instanton calculus, one can expand $f(\tau, v, m)$ in small $m$, and we can compare $f$ and $f_{\text {S-dual }}$ at $m^{4}$ order. We find that

$$
\left(f-N f_{\mathrm{U}(1)}\right)-\left.f_{\mathrm{S}-\text { dual }}\right|_{m^{4}}=\frac{N^{3}-N}{288} m^{4} E_{2}(\tau),
$$

which we checked till $q^{2}$ order for general $N$, and till $q^{3}$ for $N=2,3$. Therefore, we find that the microscopic prepotential is compatible with S-duality at $m^{4}$ order, up to the addition of an 'anomalous' term on the right hand side independent of the Coulomb VEV.

One can make further recursive calculations of $f_{n}$ for $n \geq 3$, using (2.72), and test the consistency of $f_{\text {S-dual }}$ with our microscopic $f$. The next recursion relation of $(2.72)$ is

$$
\frac{\partial f_{3}}{\partial E_{2}}=-\frac{1}{12} \frac{\partial f_{1}}{\partial v} \frac{\partial f_{2}}{\partial v}
$$

Knowing $f_{1}, f_{2}$, one can integrate $(2.83)$ to obtain

$$
\begin{aligned}
f_{3}= & -\frac{E_{2}(\tau)^{2}}{1152}\left[\sum_{\alpha \in \Delta}\left(2 \mathrm{Li}_{-3}\left(e^{\alpha(v)}\right)-4 \mathrm{Li}_{-1}\left(e^{\alpha(v)}\right)^{2}\right)+2 \sum_{\alpha \in \Delta} \sum_{\beta \in \Psi(\alpha)} \operatorname{Li}_{0}\left(e^{\alpha(v)}\right) \mathrm{Li}_{-2}\left(e^{\beta(v)}\right)\right] \\
& +c_{3}(v) E_{4}(\tau) .
\end{aligned}
$$

The integration constant $c_{3}(v)$ can be determined by expanding $f_{3}$ in $q$, and comparing the $q^{0}$ order with the perturbative contribution $\left(f-N f_{\mathrm{U}(1)}\right)_{\text {pert }}$ at $m^{6}$ order. One obtains

$c_{3}(v)=\frac{1}{2880} \sum_{\alpha \in \Delta} \mathrm{Li}_{-3}\left(e^{\alpha(v)}\right)-\frac{1}{288} \sum_{\alpha \in \Delta} \operatorname{Li}_{-2}\left(e^{\alpha(v)}\right)^{2}+\frac{1}{576} \sum_{\alpha \in \Delta} \sum_{\beta \in \Psi(\alpha)} \operatorname{Li}_{0}\left(e^{\alpha(v)}\right) \operatorname{Li}_{-2}\left(e^{\beta(v)}\right)$.

Inserting this $c_{3}(v)$ in (2.84), one can further study the higher order coefficients of $f_{3}$ in $q$ expansion, against the microscopic result $f$. We find that

$$
\left(f-N f_{\mathrm{U}(1)}\right)-\left.f_{\mathrm{S}-\text { dual }}\right|_{m^{6}}=0
$$

which we checked till $q^{2}$ order for general $N$, and till $q^{3}$ order for $N=2,3$.

Integrating (2.72) to get higher $f_{n}$ 's, the integration constants take the following form,

$$
f_{n}(\tau, v) \leftarrow \sum_{4 a+6 b=2 n-2, a \geq 0, b \geq 0} c_{a, b}(v) E_{4}(\tau)^{a} E_{6}(\tau)^{b} .
$$

More concretely, one would get

$$
f_{4} \leftarrow c_{0,1} E_{6}, f_{5} \leftarrow c_{2,0} E_{4}^{2}, f_{6} \leftarrow c_{1,1} E_{4} E_{6}, f_{7} \leftarrow c_{3,0} E_{4}^{3}+c_{0,2} E_{6}^{2}, f_{8} \leftarrow c_{2,1} E_{4}^{2} E_{6}, \cdots
$$

and so on. To fix the coefficients $c_{p, q}(v)$, one should use some low order data of $f-N f_{\mathrm{U}(1)}$. If there are $k+1$ independent $c_{p, q}$ 's, one should use up to $k$ instanton coefficients of 
$f-N f_{\mathrm{U}(1)}$ to fix them. Then from $k+1$ or higher instantons, one can test $f_{n}$ by comparing with $f-N f_{\mathrm{U}(1)}$. For general $N$, we tested $f_{\mathrm{S}-\text { dual }}$ against $f-N f_{\mathrm{U}(1)}$ till $m^{6}$ and $q^{2}$ orders, as already reported above. For $\mathrm{U}(2)$, we tested it till $m^{14}$ (i.e. $f_{7}$ ) and $q^{3}$ orders. For $\mathrm{U}(3)$, we tested it till $m^{8}$ (i.e. $f_{4}$ ) and $q^{3}$ orders. The result is that there is no further difference between $f_{\mathrm{S}-\text { dual }}$ and $f-N f_{\mathrm{U}(1)}$ from $m^{6}$ and higher orders. Namely, we find that

$$
f(\tau, v, m)=f_{\mathrm{S}-\mathrm{dual}}(\tau, v, m)+N f_{\mathrm{U}(1)}(\tau, m)+\frac{N^{3}-N}{288} m^{4} E_{2}(\tau) .
$$

The S-duality transformation of $f_{\mathrm{U}(1)}$ can be separately derived from (2.31), which is

$$
\begin{aligned}
\tau^{2} f_{\mathrm{U}(1)}(-1 / \tau, m / \tau)-f_{\mathrm{U}(1)}(\tau, m) & =\frac{m^{2}}{2} \log \frac{\phi(-1 / \tau)^{2}}{\tau \phi(\tau)^{2}}+\frac{m^{4}}{288}\left(\tau^{-2} E_{2}(-1 / \tau)-E_{2}(\tau)\right) \\
& =\frac{m^{2}}{2}\left[\frac{\pi i \tau}{6}+\frac{\pi i}{6 \tau}+\log (-i)\right]+\frac{m^{4}}{48 \pi i \tau}
\end{aligned}
$$

where $\eta(-1 / \tau)=\sqrt{-i \tau} \eta(\tau)$. Although we checked (2.89) in a serious expansion in small $m$, we believe it is an exact property, valid for finite $m$. In section 2.2 , we shall provide another test of (2.89) by keeping $m$ finite, but expanding $f$ as a series of $e^{-v}$ at positive Coulomb VEV $v$. Also, in section 2.3, we extend (2.89) to all ADE theories.

\subsection{Derivation from M-strings}

In this subsection, we provide another derivation of the S-duality of prepotential. The analysis here will provide more evidence for our S-duality anomaly at finite $m$. It may also provide strong hints towards the S-duality of the partition function $Z\left(\tau, v, m, \epsilon_{1,2}\right)$ at finite $\epsilon_{1,2}$, but we postpone more extensive discussions on $Z$ to a separate project.

We use an alternative partition function $\check{Z}\left(\tau, v, m, \epsilon_{1,2}\right)=\check{Z}_{\text {pert }} Z_{\text {inst. }}$. This differs from $Z$ by a different perturbative partition function $\check{Z}_{\text {pert }}$. For the purpose of studying the prepotential in the limit $\epsilon_{1,2} \rightarrow 0$, we already saw that they differ only by a trivial term, as shown in (2.24) in a suitable regime on the complex $v$ plane. The last term of (2.24) is separately invariant under the S-duality transformation $\left(\tau, m, \epsilon_{1,2}\right) \rightarrow\left(-\frac{1}{\tau}, \frac{m}{\tau}, \frac{\epsilon_{1,2}}{\tau}\right)$, and is independent of $v$, so it is completely fine to drop this constant factor when discussing the S-duality of prepotential. $\check{Z}$ can be expanded in $e^{-\alpha_{i}(v)}$ when $\alpha_{i}(v)$ are positive and sufficiently large, where $\alpha_{i}(i=1, \cdots, N-1)$ are the simple roots of $\mathrm{SU}(N)$. The expansion takes the form of $[8]$

$$
\begin{aligned}
Z\left(\tau, v, m, \epsilon_{1,2}\right) & =e^{-\varepsilon_{0}} Z_{\mathrm{U}(1)}\left(\tau, m, \epsilon_{1,2}\right)^{N} \sum_{n_{1}, \cdots, n_{N-1}=0}^{\infty} e^{-\sum_{i=1}^{N-1} n_{i} \alpha_{i}(v)} Z_{\left(n_{i}\right)}\left(\tau, m, \epsilon_{1,2}\right) \\
& \equiv e^{-\varepsilon_{0}} Z_{\mathrm{U}(1)}^{N} \hat{Z} .
\end{aligned}
$$

$Z_{\mathrm{U}(1)}^{N}$ is for the D0-branes unbound to the W-bosons, just bound to one of the $N$ D4-branes, which is this independent of $e^{-\alpha_{i}(v)}$. $\varepsilon_{0}$ is defined at the beginning of section 2 by

$$
\varepsilon_{0}=\frac{m^{2}-\epsilon_{+}^{2}}{2 \epsilon_{1} \epsilon_{2}} \sum_{\alpha>0}(\alpha(v)+\pi i) .
$$


This factor has to be multiplied to guarantee (2.24). This expansion is reliable in a particular Weyl chamber of the Coulomb branch, with sufficiently large $\alpha_{i}(v)$ for all simple roots. The coefficient $Z_{\left(n_{i}\right)}\left(\tau, m, \epsilon_{1,2}\right)$ at given self-dual string numbers $n_{i}$ is computed from 'M-strings,' which are M2-branes suspended between separated M5-branes. $Z_{\left(n_{i}\right)}$ is the elliptic genus of the $2 \mathrm{~d}$ quiver gauge theory with $\mathrm{U}\left(n_{1}\right) \times \cdots \mathrm{U}\left(n_{N-1}\right)$ gauge group. See [20] for the details. $Z_{\left(n_{i}\right)}\left(\tau, m, \epsilon_{1,2}\right)$ is given by [8] (we follow the notations of [21])

$$
Z_{\left(n_{i}\right)}=\sum_{Y_{1}, \cdots, Y_{N-1} ;\left|Y_{i}\right|=n_{i}} \prod_{i=1}^{N} \prod_{s \in Y_{i}} \frac{\theta_{1}\left(\tau \mid \frac{E_{i, i+1}(s)-m+\epsilon_{-}}{2 \pi i}\right) \theta_{1}\left(\tau \mid \frac{E_{i, i-1}(s)+m+\epsilon_{-}}{2 \pi i}\right)}{\theta_{1}\left(\tau \mid \frac{E_{i, i}(s)+\epsilon_{1}}{2 \pi i}\right) \theta_{1}\left(\tau \mid \frac{E_{i, i}(s)-\epsilon_{2}}{2 \pi i}\right)},
$$

where $s=(a, b)$ denotes the position of each box in the Young diagram, and

$$
E_{i, j}(s=(a, b))=\left(Y_{i, a}-b\right) \epsilon_{1}-\left(Y_{j, b}^{T}-a\right) \epsilon_{2} .
$$

$Y_{i, a}$ is the length of the $a^{\prime}$ th row of $Y_{i}$, and $Y_{j, b}^{T}$ is the length of the $b^{\prime}$ th column of $Y_{j} . Y_{0}$ and $Y_{N}$ are empty by definition.

We study the S-duality of $\check{Z}$ in this setting. By using

$$
\frac{\theta_{1}\left(-\frac{1}{\tau}, \frac{z}{\tau}\right)}{\eta\left(-\frac{1}{\tau}\right)}=e^{\frac{\pi i z^{2}}{\tau}} \frac{\theta_{1}(\tau, z)}{\eta(\tau)}
$$

one can show that $Z_{\left(n_{i}\right)}$ transforms as

$Z_{\left(n_{i}\right)}\left(-\frac{1}{\tau}, \frac{m}{\tau}, \frac{\epsilon_{1,2}}{\tau}\right)=\exp \left[\frac{1}{4 \pi i \tau}\left(\epsilon_{1} \epsilon_{2} \sum_{i, j=1}^{N-1} A_{i j} n_{i} n_{j}+2\left(m^{2}-\epsilon_{+}^{2}\right) \sum_{i=1}^{N-1} n_{i}\right)\right] Z_{\left(n_{i}\right)}\left(\tau, m, \epsilon_{1,2}\right)$.

$A_{i j}$ is the Cartan matrix for $\mathrm{SU}(N)$, given by $A_{i i}=2, A_{i, i+1}=A_{i, i-1}=-1$ and $A_{i j}=0$ otherwise. This transformation can be expressed as a modular anomaly equation, as follows. First note that Jacobi's theta functions can be expressed as

$$
\theta_{1}(\tau \mid z)=2 \pi i z \eta(\tau)^{3} \exp \left[\sum_{k=1}^{\infty} \frac{B_{2 k}}{(2 k)(2 k) !} E_{2 k}(\tau)(2 \pi i z)^{2 k}\right] .
$$

$\tau$ dependence of $Z_{\left(n_{i}\right)}$ can be understood as its dependence through $E_{2 n}(\tau)$, since $\eta(\tau)^{3}$ factors cancel out in (2.93). As we did in section 2.1 for the prepotential, the $\tau$ dependence of $Z_{\left(n_{i}\right)}$ can again be decomposed into its dependence through $E_{2}(\tau)$, and the rest. To emphasize this, we write $Z_{\left(n_{i}\right)}\left(\tau, m, \epsilon_{1,2}, E_{2}\right)$. In this setting, the modular anomaly $e^{\frac{\pi i z^{2}}{\tau}}$ of (2.95) appears due to the dependence of $\theta_{1}$ on $E_{2}(\tau)$, which is not modular. Therefore, we can rephrase $(2.96)$ as

$$
\frac{\partial}{\partial E_{2}} Z_{\left(n_{i}\right)}\left(\tau, m, \epsilon_{1,2} ; E_{2}\right)=\frac{1}{24}\left(\epsilon_{1} \epsilon_{2} \sum_{i, j=1}^{N-1} A_{i j} n_{i} n_{j}+2\left(m^{2}-\epsilon_{+}^{2}\right) \sum_{i=1}^{N-1} n_{i}\right) Z_{\left(n_{i}\right)} .
$$

Let us consider $\hat{Z}\left(\tau, \lambda, m, \epsilon_{1,2} ; E_{2}\right)=\sum_{n_{i}=0}^{\infty} e^{-n_{i} \lambda_{i}} Z_{\left(n_{i}\right)}\left(\tau, m, \epsilon_{1,2} ; E_{2}\right)$, where we defined $\lambda_{i} \equiv v_{i}-v_{i+1}>0$ with the choice of simple roots $\alpha_{i}=e_{i}-e_{i+1}$. Here we view $\hat{Z}$ as a 
function of $\lambda$ 's, since it depends only on the differences of $v$ 's. Now with the replacement $n_{i} \rightarrow-\frac{\partial}{\partial \lambda_{i}}$ in the Laplace transformation, one obtains

$$
\frac{\partial}{\partial E_{2}} \hat{Z}\left(\tau, \lambda, m, \epsilon_{1,2} ; E_{2}\right)=\frac{1}{24}\left(\epsilon_{1} \epsilon_{2} \sum_{i, j=1}^{N-1} A_{i j} \frac{\partial^{2}}{\partial \lambda_{i} \partial \lambda_{j}}-2\left(m^{2}-\epsilon_{+}^{2}\right) \sum_{i=1}^{N-1} \frac{\partial}{\partial \lambda_{i}}\right) \hat{Z} .
$$

This is essentially the 'holomorphic anomaly equation', eq. (3.54) of [8]. In our context, we continue to study it as the modular anomaly equation.

We shall get better intuitions on the modular anomaly equation. Consider $\tilde{Z} \equiv e^{-\varepsilon_{0}} \hat{Z}$ with $\varepsilon_{0}=\frac{m^{2}-\epsilon_{+}^{2}}{2 \epsilon_{1} \epsilon_{2}} \sum_{\alpha>0}(\alpha(v)+\pi i)$, still without $\left(Z_{\mathrm{U}(1)}\right)^{N}$ included. Since the $v$ independent part of $\varepsilon_{0}$ is independent of $E_{2}, \lambda$, it does not affect (2.99). The $v$ dependent part can be written as

$$
\sum_{\alpha>0} \alpha(v)=\sum_{i<j}\left(v_{i}-v_{j}\right)=\sum_{i=1}^{N}[(N-i)-(i-1)] v_{i}=\sum_{i=1}^{N}(N+1-2 i) v_{i} .
$$

Since $\frac{\partial \lambda_{i}(v)}{\partial v_{j}}=\delta_{i, j}-\delta_{i+1, j}, \frac{\partial}{\partial v_{j}}$ acting on a function of $\lambda_{i}$ 's can be written as $\frac{\partial}{\partial v_{j}}=\frac{\partial \lambda_{i}(v)}{\partial v_{j}} \frac{\partial}{\partial \lambda_{i}}$,

$$
\frac{\partial}{\partial v_{1}}=\frac{\partial}{\partial \lambda_{1}}, \frac{\partial}{\partial v_{j}}=\frac{\partial}{\partial \lambda_{j}}-\frac{\partial}{\partial \lambda_{j-1}} \quad(j=2, \cdots, N-1), \frac{\partial}{\partial v_{N}}=-\frac{\partial}{\partial \lambda_{N-1}} .
$$

The Laplacian of $v$ acting on a function of $\lambda$ is given by

$$
\nabla^{2} \equiv \sum_{i=1}^{N} \frac{\partial^{2}}{\partial v_{i}^{2}}=A_{i j} \frac{\partial^{2}}{\partial \lambda_{i} \partial \lambda_{j}}
$$

One also finds that

$$
\sum_{i=1}^{N-1} \frac{\partial}{\partial \lambda_{i}}=\sum_{i=1}^{N}(a-i) \partial_{v_{i}}
$$

for any number $a$. Acting $\nabla^{2}$ on $\tilde{Z}=e^{-\varepsilon_{0}} \hat{Z}$, one obtains

$$
\begin{aligned}
\nabla^{2} \tilde{Z} & =e^{-\varepsilon_{0}} \nabla^{2} \hat{Z}-2 \frac{m^{2}-\epsilon_{+}^{2}}{2 \epsilon_{1} \epsilon_{2}} e^{-\varepsilon_{0}} \sum_{i=1}^{N}(N+1-2 i) \partial_{v_{i}} \hat{Z}+\left(\frac{m^{2}-\epsilon_{+}^{2}}{2 \epsilon_{1} \epsilon_{2}}\right)^{2} e^{-\varepsilon_{0}} \sum_{i=1}^{N}(2 i-N-1)^{2} \hat{Z} \\
& =e^{-\varepsilon_{0}}\left[\nabla^{2} \hat{Z}-2 \frac{m^{2}-\epsilon_{+}^{2}}{\epsilon_{1} \epsilon_{2}} \sum_{i=1}^{N-1} \frac{\partial}{\partial \lambda_{i}} \hat{Z}+\frac{N^{3}-N}{3}\left(\frac{m^{2}-\epsilon_{+}^{2}}{2 \epsilon_{1} \epsilon_{2}}\right)^{2} \hat{Z}\right]
\end{aligned}
$$

where we used $\frac{\partial \varepsilon_{0}}{\partial v_{i}}=\frac{m^{2}-\epsilon_{+}^{2}}{2 \epsilon_{1} \epsilon_{2}}(N+1-2 i)$. Using (2.99), this can be rewritten as

$$
\nabla^{2} \tilde{Z}=e^{-\varepsilon_{0}}\left[\frac{24}{\epsilon_{1} \epsilon_{2}} \frac{\partial}{\partial E_{2}} \hat{Z}+\frac{N^{3}-N}{3}\left(\frac{m^{2}-\epsilon_{+}^{2}}{2 \epsilon_{1} \epsilon_{2}}\right)^{2} \hat{Z}\right] .
$$

Thus, one finds that the partition function

$$
Z_{\text {S-dual }} \equiv \exp \left[\frac{\epsilon_{1} \epsilon_{2}}{24} \frac{N^{3}-N}{12}\left(\frac{m^{2}-\epsilon_{+}^{2}}{\epsilon_{1} \epsilon_{2}}\right)^{2} E_{2}\right] \tilde{Z}=\exp \left[\frac{N^{3}-N}{288} \frac{\left(m^{2}-\epsilon_{+}^{2}\right)^{2}}{\epsilon_{1} \epsilon_{2}} E_{2}-\varepsilon_{0}\right] \hat{Z}
$$


satisfies the modular anomaly equation

$$
\frac{\partial}{\partial E_{2}} Z_{\mathrm{S} \text {-dual }}\left(\tau, v, m, \epsilon_{1,2} ; E_{2}\right)=\frac{\epsilon_{1} \epsilon_{2}}{24} \nabla^{2} Z_{\mathrm{S}-\text { dual }} .
$$

Also, from the M-string expansion form of $\hat{Z}$, and the form of the prefactors we multiplied to define $Z_{\mathrm{S} \text {-dual }}, Z_{\mathrm{S} \text {-dual }}$ satisfies the following quasi-modularity condition,

$$
Z_{\mathrm{S} \text {-dual }}\left(-\frac{1}{\tau}, v, \frac{m}{\tau}, \frac{\epsilon_{1,2}}{\tau} ; E_{2}\left(-\frac{1}{\tau}\right)\right)=Z_{\mathrm{S}-\text { dual }}\left(\tau, v, m, \epsilon_{1,2}, E_{2}(\tau)+\delta\right)
$$

where $\delta=\frac{6}{\pi i \tau}$. (2.107) and (2.108) are the two main properties of $Z_{\mathrm{S} \text {-dual }}$.

Using (2.107) and (2.108), we would like to study the relation between $Z_{\mathrm{S} \text {-dual }}\left(\tau, v, m, \epsilon_{1,2}, E_{2}\right)$ and $Z_{\mathrm{S} \text {-dual }}\left(-\frac{1}{\tau}, v, \frac{m}{\tau}, \frac{\epsilon_{1,2}}{\tau}, E_{2}\left(-\frac{1}{\tau}\right)\right)$. Since $\tilde{Z}$ is related to $Z_{\mathrm{S} \text {-dual }}$ in a simple manner, answering this question will tell us how $\tilde{Z}$ transforms under S-duality. Then, since we already understand how the $\mathrm{U}(1)^{N}$ part $Z_{\mathrm{U}(1)}^{N}$ transforms under S-duality [38], we shall in turn know the S-duality transformation of $\check{Z}$. Using (2.108), we should understand how $Z_{\mathrm{S} \text {-dual }}\left(\tau, v, m, \epsilon_{1,2} ; E_{2}(\tau)+\delta\right)$ and $Z_{\mathrm{S} \text {-dual }}\left(\tau, v, m, \epsilon_{1,2} ; E_{2}\right)$ are related to each other, at same values of $\tau, v, m, \epsilon_{1,2}$ but with a shift of $E_{2}$ by $\delta=\frac{6}{\pi i \tau}$. Let us formally regard $E_{2}$ as time variable, and $Z_{\mathrm{S} \text {-dual }}$ as a wavefunction on the space formed by $v_{i}$. Then (2.107) takes the form of heat equation, or Euclidean Schrödinger equation for a free particle. More precisely, taking $\tau$ to be purely imaginary for simplicity, $\delta$ is real and negative. So $-E_{2}$ plays the role of time, and we would like to understand the time evolution $Z_{\mathrm{S} \text {-dual }}\left(E_{2}+\delta\right)$ by $-\delta$ following the heat equation. The heat equation comes with proper sign when $\epsilon_{1} \epsilon_{2}<0$. In this case, the time evolution is described by evolving $Z_{\mathrm{S} \text {-dual }}\left(E_{2}\right)$ by the Gaussian heat kernel as

$$
Z_{\mathrm{S} \text {-dual }}\left(\tau, v, m, \epsilon_{1,2} ; E_{2}(\tau)+\delta\right)=\int_{-\infty}^{\infty} \prod_{i=1}^{N} d v_{i}^{\prime} K\left(v, v^{\prime}\right) Z_{\mathrm{S} \text {-dual }}\left(\tau, v^{\prime}, m, \epsilon_{1,2} ; E_{2}(\tau)\right),
$$

where

$$
K\left(v, v^{\prime}\right)=\left(\frac{i \tau}{\epsilon_{1} \epsilon_{2}}\right)^{\frac{N}{2}} \exp \left[-\frac{\pi i \tau}{\epsilon_{1} \epsilon_{2}}\left(v-v^{\prime}\right)^{2}\right]
$$

is the heat kernel which approaches $K\left(v, v^{\prime}\right) \rightarrow \delta^{(N)}\left(v-v^{\prime}\right)$ when $\frac{i \tau}{\epsilon_{1} \epsilon_{2}} \rightarrow 0$. When $\epsilon_{1} \epsilon_{2}>0$, the 'time evolution' from $Z_{\mathrm{S} \text {-dual }}\left(E_{2}\right)$ to $Z_{\mathrm{S} \text {-dual }}\left(E_{2}+\delta\right)$ is described by the time reversal of the heat equation. Therefore, one finds

$$
Z_{\mathrm{S} \text {-dual }}\left(\tau, v, m, \epsilon_{1,2} ; E_{2}(\tau)\right)=\int_{-\infty}^{\infty} \prod_{i=1}^{N} d v_{i}^{\prime} K_{-}\left(v, v^{\prime}\right) Z_{\mathrm{S} \text {-dual }}\left(\tau, v^{\prime}, m, \epsilon_{1,2} ; E_{2}(\tau)+\delta\right)
$$

with $K_{-}\left(v, v^{\prime}\right)=\left(-\frac{i \tau}{\epsilon_{1} \epsilon_{2}}\right)^{\frac{N}{2}} \exp \left[\frac{\pi i \tau}{\epsilon_{1} \epsilon_{2}}\left(v-v^{\prime}\right)^{2}\right]$ for $\epsilon_{1} \epsilon_{2}>0$.

The S-duality of $f_{\text {S-dual }}$ can be studied from (2.109) or (2.111) by a saddle point approximation of the $v^{\prime}$ integral at $\epsilon_{1,2} \rightarrow 0$. Using both equations yield identical results. One finds

$$
Z_{\mathrm{S} \text {-dual }}=e^{-\frac{f_{\mathrm{S} \text {-dual }}}{\epsilon_{1} \epsilon_{2}}} \sim \exp \left[-\frac{\hat{f}}{\epsilon_{1} \epsilon_{2}}+\frac{N^{3}-N}{288} \frac{m^{4}}{\epsilon_{1} \epsilon_{2}} E_{2}(\tau)-\frac{m^{2}}{2 \epsilon_{1} \epsilon_{2}} \sum_{\alpha>0}(\alpha(v)+\pi i)\right]
$$


in the limit $\epsilon_{1,2} \rightarrow 0$, so that

$$
f_{\text {S-dual }}=\hat{f}-\frac{N^{3}-N}{288} m^{4} E_{2}(\tau)+\frac{m^{2}}{2} \sum_{\alpha>0}(\alpha(v)+\pi i) .
$$

We shall show that $f_{\mathrm{S} \text {-dual }}$ defined this way is the same $f_{\mathrm{S} \text {-dual }}$ defined and computed in section 2.1. Firstly, note that

$$
\hat{f}=\check{f}-N f_{\mathrm{U}(1)}-\frac{m^{2}}{2} \sum_{\alpha<0}(\alpha(v)+\pi i)=f-N f_{\mathrm{U}(1)}-\frac{m^{2}}{2} \sum_{\alpha<0}(\alpha(v)+\pi i)-\frac{\pi i m^{2}\left|\Delta_{+}\right|}{2}
$$

from the relations $\check{Z}=e^{-\varepsilon_{0}}\left[Z_{\mathrm{U}(1)}\right]^{N} \hat{Z}$ and $\check{Z} \sim Z e^{\frac{\pi i m^{2}\left|\Delta_{+}\right|}{2 \epsilon_{1} \epsilon_{2}}}$. Inserting this in (2.113), one obtains

$$
f_{\mathrm{S}-\text { dual }}=f-N f_{\mathrm{U}(1)}-\frac{N^{3}-N}{288} m^{4} E_{2}(\tau)-\frac{\pi i m^{2}\left|\Delta_{+}\right|}{2} .
$$

This is completely the same as the relation between $f$ and $f_{\text {S-dual }}$ that we found in section 2.1, except the last term on the right hand side. However, we know that the last term comes from using slightly different perturbative partition function in $\check{Z},(2.24)$. Also this term can be completely ignored for studying S-duality since it is separately invariant under S-duality. Therefore, after discarding this last term, we find that $f_{\mathrm{S} \text {-dual }}$ is the same as $f_{\mathrm{S} \text {-dual }}$ defined in section 2.1. Now one can independently check that $f_{\mathrm{S} \text {-dual }}$ is S-dual. Firstly, Since $Z_{\mathrm{S} \text {-dual }}$ is quasi-modular, so is $f_{\mathrm{S} \text {-dual }}$, i.e.

$$
\tau^{2} f_{\mathrm{S}-\mathrm{dual}}\left(-\frac{1}{\tau}, v, \frac{m}{\tau}, E_{2}(-1 / \tau)\right)=f_{\mathrm{S}-\mathrm{dual}}\left(\tau, v, m, E_{2}(\tau)+\delta\right)
$$

Secondly, inserting $Z_{\text {S-dual }} \sim \exp \left[-\frac{f_{\text {S-dual }}}{\epsilon_{1} \epsilon_{2}}\right]$ into $(2.107)$ and keeping the leading terms in the limit $\epsilon_{1,2} \rightarrow 0$, one finds $\frac{\partial f_{\mathrm{S} \text {-dual }}}{\partial E_{2}}=-\frac{1}{24}\left(\frac{\partial f_{\mathrm{S} \text {-dual }}}{\partial v}\right)^{2}$, the same modular anomaly equation that we studied in section 2.1 [22]. The last two equations guarantee the S-duality of $f_{\mathrm{S} \text {-dual }}$, completing an alternative proof of S-duality based on M-strings.

While making an alternative derivation of the S-duality and its anomaly, we did not assume the smallness of $m$. On the other hand, around (2.24), we required $\operatorname{Re}(\alpha(v))>$ $\pm \operatorname{Re}(m)$ for the positive roots $\alpha$, and that $\operatorname{Im}(\alpha(v) \pm m)$ for positive roots be in the range $(0,2 \pi]$, to justify the uses of $\check{F}_{\text {pert }}$ and $\check{Z}_{\text {pert }}$. So at least in this range, $F_{\text {anom }}=$ $N f_{\mathrm{U}(1)}+\frac{N^{3}-N}{288} m^{4} E_{2}(\tau)$ is exact at finite $m$. One can scan the whole complex planes of $v$ and $m$, considering the multiple values of $\mathrm{Li}_{3}$ function, to find the most general form of the S-duality anomaly when (2.24) is violated. We shall not do this exercise here.

Before closing this subsection, we comment on the nature of the S-duality transformation of $Z_{\mathrm{S} \text {-dual }}$ or $\check{Z}$, and what it implies to the S-duality of $Z$. This issue is also related to the S-duality of the $4 \mathrm{~d}$ limit of $Z$, which was studied in the context of AGT correspondence [23]. Taking the $4 \mathrm{~d}$ limit $R \rightarrow 0$ with fixed $a, M, \varepsilon_{1,2}, m, \tau,(2.109)$ asserts that $Z^{4 \mathrm{~d}}\left(\tau, a, m, \varepsilon_{1,2}\right)$ is related to its S-dual by a Gaussian S-duality kernel. This is because the $R$ dependence $K$ is given by

$$
K\left(a, a^{\prime}\right)=R^{-N}\left(\frac{i \tau}{\varepsilon_{1} \varepsilon_{2}}\right)^{\frac{N}{2}} \exp \left[-\frac{\pi i \tau}{\varepsilon_{1} \varepsilon_{2}}\left(a-a^{\prime}\right)^{2}\right]
$$


and the overall $R$ dependence is absorbed into the $v^{\prime}$ integration to be $\prod_{i} d a_{i}^{\prime}$. Expanding the exponent of the kernel, and inserting $a=\frac{a_{D}}{\tau}$, one obtains

$$
\exp \left[-\frac{F_{\mathrm{cl}}\left(a^{\prime}, \tau\right)-F_{\mathrm{cl}}\left(a_{D},-\frac{1}{\tau}\right)}{\varepsilon_{1} \varepsilon_{2}}\right] \exp \left[\frac{\pi i a_{D} a^{\prime}}{\varepsilon_{1} \varepsilon_{2}}\right],
$$

where $F_{\mathrm{cl}}(a, \tau)=\pi i \tau a^{2}$. The two $F_{\mathrm{cl}}$ 's can be absorbed into two $Z^{4 \mathrm{~d}}$ 's on the left and right hand sides of (2.109). Then, the $4 \mathrm{~d}$ limit of (2.109) states that S-dualization is Fourier transformation. Our studies imply that the same result holds for $Z_{\mathrm{S}-\text { dual }}$ in $6 \mathrm{~d}$.

In fact, the proper S-duality transformation of the $4 \mathrm{~d}$ partition function is known not to be the Fourier transformation. Instead, based on the AGT correspondence, the S-duality kernel is asserted to be a nontrivial function given by the partition function of the $3 \mathrm{~d} T[\mathrm{SU}(N)]$ theory on $S^{3}[24,25]$. On the other hand, it has been found that the S-duality of the Omega deformed partition function is the Fourier transformation at all perturbative orders in $\varepsilon_{1}, \varepsilon_{2}[15,22,26,27]$. An explanation of this was given in [26], which finds that the choice of proper 'normalization factor' independent of $\tau$ dressing the instanton partition function yields such a nonperturbative correction in $\varepsilon_{1,2}$. This is the 'choice' of $Z_{\text {pert }}^{4 \mathrm{~d}}$, which was called $N(a)$ and $N_{s}(a)$ in [26]. Incidently, what we find in $6 \mathrm{~d}$ is analogous to the findings of [26]. Namely, $Z$ was defined in section 2 with $Z_{\text {pert }}$ which is manifestly invariant under Weyl symmetry. However, $\check{Z}$ was defined with $\check{Z}_{\text {pert }}$ which is not invariant under Weyl symmetry. In fact, we checked that the ratio $\frac{\check{Z}_{\text {pert }}}{Z_{\text {pert }}}$ at small $\epsilon_{1,2}$ is nonperturbative in $\epsilon_{1,2}$, which is qualitatively consistent with [26]. So along this line, it will be interesting to pursue the $6 \mathrm{~d}$ extensions of [26]. We stress again that, all our findings in this subsection concerns the prepotential in the $\epsilon_{1,2} \rightarrow 0$ limit, for which the distinction of $Z_{\text {pert }}$ or $\check{Z}_{\text {pert }}$ is irrelevant.

\section{$2.36 \mathrm{~d}(2,0)$ theories of $D_{N}$ and $E_{N}$ types}

We generalize some studies we made for $A_{N-1}$ type $(2,0)$ theories to $D_{N}$ and $E_{N}$ type theories. For $D_{N}$ type theories, $f_{\text {S-dual }}$ can be compared with microscopic instanton calculus for the $5 \mathrm{~d} \mathrm{SO}(2 N) \mathcal{N}=1^{*}$ theory, or the D0-D4-O4 matrix quantum mechanics [9]. For $E_{N}$ types, we make a prediction of the S-duality and our knowledge of 5 d perturbative prepotential. All ADE results will be partly tested in section 3.2 from $6 \mathrm{~d}$ chiral anomalies.

In the setting of section 2.1 , the leading coefficient $f_{1}$ of $f_{\text {S-dual }}=\sum_{n=1}^{\infty} m^{2 n} f_{n}$ is obtained from $\left(f-r f_{\mathrm{U}(1)}\right)_{\text {pert }}$, where $r$ is the rank of the gauge group. The result is

$$
f_{1}=\frac{1}{2} \sum_{\alpha \in \Delta} \log \left(1-e^{\alpha(v)}\right) .
$$

Then using (2.72) at $n=2$, one finds

$$
f_{2}=-\frac{E_{2}(\tau)}{96} \sum_{\alpha, \beta \in \Delta} \frac{\alpha \cdot \beta}{\left(1-e^{\alpha(v)}\right)\left(1-e^{\beta(v)}\right)} .
$$


To proceed, we classify the roots $\beta$ depending on their norm with $\alpha$. The possibilities are

$$
\begin{aligned}
& \text { (1) }: \alpha \cdot \beta= \pm 2 \text { if } \pm \beta=\alpha \\
& (2): \alpha \cdot \beta= \pm 1 \text { if } \pm \beta \in \Psi(\alpha) \\
& (3): \alpha \cdot \beta=0 \text { otherwise } .
\end{aligned}
$$

It is again important to understand the set $\Psi(\alpha)$ for ADE, which we explain now.

For $D_{N}=\mathrm{SO}(2 N)$, the $2 N^{2}-2 N$ roots in $\Delta$ are given by $\pm e_{i} \pm e_{j}$, where $i, j=1, \cdots, N$ and $i<j$. Elements of $\Psi(\alpha)$ are given for various $\alpha$ by

$$
\begin{aligned}
& \alpha=e_{i}-e_{j}: \Psi(\alpha)=\left\{k \neq i, j: e_{i} \pm e_{k}, \pm e_{k}-e_{j}\right\}, \quad 4(N-2) \text { elements } \\
& \alpha=e_{i}+e_{j}: \Psi(\alpha)=\left\{k \neq i, j: e_{i} \pm e_{k}, e_{j} \pm e_{k}\right\}, \quad 4(N-2) \text { elements. }
\end{aligned}
$$

For $E_{6}$, the number of roots is $|\Delta|=72.40$ roots take the form of $\pm e_{i} \pm e_{j}$ where $i \neq j$ and $i, j=1, \cdots, 5$, from the $\mathrm{SO}(10)$ subalgebra. Additional 32 roots take the form of $\pm \frac{1}{2}\left( \pm e_{1} \pm \cdots \pm e_{5}-e_{6}-e_{7}+e_{8}\right)$, where the total number of - signs is even. The structure of $\Psi(\alpha)$ is given for various $\alpha$ as follows. Firstly, when $\alpha=e_{i}-e_{j}$, then

$$
\Psi(\alpha)=\left\{k \neq i, j: e_{i} \pm e_{k}, \pm e_{k}-e_{j}\right\} \cup\left\{\frac{1}{2}\left(e_{i}-e_{j}+\cdots\right)\right\}
$$

where $\cdots$ means that all possible signs are allowed in the 32 spinorial elements. Thus, one finds $12+8=20$ elements of $\Psi(\alpha)$ in this case. Similarly, for $\alpha=e_{i}+e_{j}$, one finds

$$
\Psi(\alpha)=\left\{k \neq i, j: e_{i} \pm e_{k}, e_{j} \pm e_{k}\right\} \cup\left\{\frac{1}{2}\left(e_{i}+e_{j}+\cdots\right)\right\}
$$

where $\cdots$ means the same. So again, one finds $|\Psi(\alpha)|=12+8=20$. For $\alpha=-e_{i}-e_{j}$, one can do a similar analysis. Finally, $\alpha$ can be one of the 32 spinorial elements, $\alpha=$ $\frac{s_{0}}{2}\left(s_{1} e_{1}+\cdots+s_{5} e_{5}-e_{6}-e_{7}+e_{8}\right)$ with $s_{0}, \cdots, s_{5}= \pm 1$ and $s_{1} \cdots s_{5}=1$. Then,

$$
\Psi(\alpha)=\left\{s_{0}\left(s_{i} e_{i}+s_{j} e_{j}\right)\right\} \cup\left\{\alpha-s_{0}\left(s_{i} e_{i}+s_{j} e_{j}\right)\right\},
$$

so $|\Psi(\alpha)|={ }_{5} C_{2}+{ }_{5} C_{2}=20$. For $E_{7},|\Delta|=126$. 60 roots take the form of $\pm e_{i} \pm e_{j}$, $i, j=1, \cdots, 6$, from $\mathrm{SO}(12)$ subalgebra. Additional 64 roots take the form of $\pm \frac{1}{2}\left( \pm e_{1} \pm\right.$ $\left.\cdots \pm e_{6}-e_{7}+e_{8}\right)$, with total number of - signs being even. Finally, 2 more roots are given by $\pm\left(e_{7}-e_{8}\right)$. When $\alpha= \pm e_{i} \pm e_{j}, \Psi(\alpha)$ takes the same structure as that shown for $E_{6}$. For instance, for $\alpha=e_{i}+e_{j}$, one finds $\Psi(\alpha)=\left\{k \neq i, j \mid e_{i} \pm e_{k}, e_{j} \pm e_{k}\right\} \cup\left\{\frac{1}{2}\left(e_{i}+e_{j} \cdots\right)\right\}$ with $|\Psi(\alpha)|=16+16=32$. When $\alpha=\frac{s_{0}}{2}\left(s_{1} e_{1}+\cdots+s_{6} e_{6}-e_{7}+e_{8}\right)$, with $s_{1} \cdots s_{6}=1$, one finds

$$
\Psi(\alpha)=\left\{s_{0}\left(s_{i} e_{i}+s_{j} e_{j}\right)\right\} \cup\left\{\alpha-s_{0}\left(s_{i} e_{i}+s_{j} e_{j}\right)\right\} \cup\left\{s_{0}\left(e_{8}-e_{7}\right), \alpha+s_{0}\left(e_{7}-e_{8}\right)\right\}
$$

with $|\Psi(\alpha)|={ }_{6} C_{2}+{ }_{6} C_{2}+2=32$. Finally, when $\alpha=e_{7}-e_{8}$, one finds

$$
\Psi(\alpha)=\left\{s_{1} \cdots s_{6}=-1 \mid \frac{1}{2}\left(s_{1} e_{1}+\cdots s_{6} e_{6}+e_{7}-e_{8}\right)\right\},
$$




\begin{tabular}{|c|c|c|c|c|c|}
\hline$G$ & $A_{N-1}$ & $D_{N}$ & $E_{6}$ & $E_{7}$ & $E_{8}$ \\
\hline$r$ & $N-1$ & $N$ & 6 & 7 & 8 \\
\hline$|G|$ & $N^{2}-1$ & $2 N^{2}-N$ & 78 & 133 & 248 \\
\hline$c_{2}$ & $N$ & $2 N-2$ & 12 & 18 & 30 \\
\hline
\end{tabular}

Table 1. Data on simply laced Lie algebras.

with $|\Psi(\alpha)|=32$. The case with $\alpha=e_{8}-e_{7}$ is similar. For $E_{8},|\Delta|=240.112$ roots take the form of $\pm e_{i} \pm e_{j}, i, j=1, \cdots, 8$, from $\mathrm{SO}(16)$ subalgebra. Additional 128 roots take the form of $\frac{1}{2}\left( \pm e_{1} \pm \cdots \pm e_{8}\right)$ with number of - signs being even, forming the $\mathrm{SO}(16)$ spinor representation. For $\alpha=e_{i}+e_{j}$, one finds $\Psi(\alpha)=\left\{k \neq i, j \mid e_{i} \pm e_{k}, e_{j} \pm e_{k}\right\} \cup\left\{\frac{1}{2}\left(e_{i}+e_{j} \cdots\right)\right\}$, with $|\Psi(\alpha)|=24+32=56$. Other cases with roots of the form $\alpha= \pm e_{i} \pm e_{j}$ can be studied similarly. For $\alpha=\frac{1}{2}\left(s_{1} e_{1}+\cdots s_{8} e_{8}\right)$ with $s_{1} \cdots s_{8}=1$, one finds

$$
\Psi(\alpha)=\left\{s_{i} e_{i}+s_{j} e_{j}\right\} \cup\left\{\alpha-\left(s_{i} e_{i}+s_{j} e_{j}\right)\right\}
$$

with $|\Psi(\alpha)|={ }_{8} C_{2}+{ }_{8} C_{2}=56$. Including the $\mathrm{SU}(N)$ case studied in section 2.1, one finds $|\Psi(\alpha)|=2 c_{2}-4$, where $c_{2}$ is the dual Coxeter number. See table 1 . Another useful fact that can be checked with all $\Psi(\alpha)$ we listed above is that, if $\beta \in \Psi(\alpha)$, then $\alpha-\beta$ is also a root. One also finds $\alpha-\beta \in \Psi(\alpha)$, since $\alpha \cdot(\alpha-\beta)=1$. So at given $\alpha$, one finds

$$
\sum_{\beta \in \Psi(\alpha)} f_{\alpha, \beta}=\sum_{\beta \in \Psi(\alpha)} f_{\alpha, \alpha-\beta}
$$

for any expression $f_{\alpha, \beta}$.

By following the analysis for the $\mathrm{U}(N)$ case, till (2.1), one finds

$$
f_{2}=-\frac{E_{2}}{96}\left[4 \sum_{\alpha \in \Delta} \operatorname{Li}_{-1}\left(e^{\alpha(v)}\right)-\left(c_{2}-3\right)(|G|-r)+\sum_{\alpha \in \Delta} \sum_{\beta \in \Psi(\alpha)} \frac{2}{\left(1-e^{\alpha(v)}\right)\left(1-e^{\beta(v)}\right)}\right] \text {. }
$$

Now we use the identity (2.129) to rewrite the last term in the parenthesis as

$$
\frac{2}{3} \sum_{\alpha \in \Delta} \sum_{\beta \in \Psi(\alpha)}\left[\frac{1}{\left(1-e^{\alpha(v)}\right)\left(1-e^{\beta(v)}\right)}+\frac{1}{\left(1-e^{-\alpha(v)}\right)\left(1-e^{\beta(v)-\alpha(v)}\right)}+\frac{1}{\left(1-e^{\alpha(v)-\beta(v)}\right)\left(1-e^{-\beta(v)}\right)}\right] .
$$

On the second term, we relabeled $\alpha$ into $-\alpha$ in the first sum, and then took $\beta-\alpha$ with $\beta \in \Psi(\alpha)$ as labeling the elements of $\Psi(-\alpha)$. The third term is simply the second term with renaming $\alpha \leftrightarrow \beta$. Using the identity $\frac{1}{\left(1-e^{x}\right)\left(1-e^{y}\right)}+\frac{1}{\left(1-e^{-x}\right)\left(1-e^{y-x}\right)}+\frac{1}{\left(1-e^{x-y}\right)\left(1-e^{-y}\right)}=$ 1, (2.131) becomes $\frac{2}{3} \sum_{\alpha \in \Delta} \sum_{\beta \in \Psi(\alpha)} 1=\frac{4}{3}(|G|-r)\left(c_{2}-2\right)$. Thus, one obtains

$$
\begin{aligned}
f_{2} & =-\frac{E_{2}(\tau)}{24}\left[\sum_{\alpha \in \Delta} \operatorname{Li}_{-1}\left(e^{\alpha(v)}\right)+\frac{1}{12}\left(c_{2}+1\right)(|G|-r)\right] \\
& =-\frac{E_{2}(\tau)}{24}\left[\sum_{\alpha \in \Delta} \operatorname{Li}_{-1}\left(e^{\alpha(v)}\right)+\frac{c_{2}|G|}{12}\right],
\end{aligned}
$$

where at the last step we used the identity $|G|=r\left(c_{2}+1\right)$ for simply-laced Lie algebra. 
$f_{2}$ contains $E_{2}(\tau)=1-24 q+\cdots$, so makes a prediction on the instanton corrections. For $G=\mathrm{SO}(2 N)$, one can compare this against microscopic instanton calculus for the $5 \mathrm{~d} \mathcal{N}=1^{*}$ theory [9]. We compared the two results at 1 instanton level for $\mathrm{SO}(8)$. Namely, (2.132) implies

$$
\left.f_{2}\right|_{q^{1}}=\left.f_{\text {S-dual }}\right|_{m^{4} q^{1}}=\sum_{\alpha \in \Delta} \operatorname{Li}_{-1}\left(e^{\alpha(v)}\right)+\frac{c_{2}|G|}{12},
$$

where $\mathrm{Li}_{-1}(x)=\frac{x}{(1-x)^{2}}$. On the other hand, the single instanton partition function $Z_{1}$ for the $\mathrm{SO}(2 N)$ theory can be obtained by starting from the Witten index for the quantum mechanics describing an $\mathrm{O}^{-}$plane, $2 \mathrm{D} 0$-branes and $2 \mathrm{~N}$ D4-branes (in the covering space). The index is a complicated residue sum. One should further subtract the contributions from D0-branes unbound to D4-O4, which was explained in [9]. Following this procedure, we checked that

$$
f_{\mathrm{S}-\text { dual }}-\left.\left(f_{\mathrm{SO}(8)}-4 f_{\mathrm{U}(1)}\right)\right|_{m^{4} q^{1}}=\frac{c_{2}|G|}{12} .
$$

One can continue to generate higher order $f_{n}$ 's, and also the microscopic instanton calculus for general $D_{N}$ at higher order in $q$, and compare them. Here we simply conjecture

$$
f(\tau, v, m)=f_{\text {S-dual }}(\tau, v, m)+r f_{\mathrm{U}(1)}(\tau, m)+\frac{c_{2}|G|}{288} m^{4} E_{2}(\tau)
$$

for all $G=\mathrm{SU}(N), \mathrm{SO}(2 N), E_{N}$, where $r$ is the rank of $G$. For $G=\mathrm{SU}(N)$, we have tested it extensively in section 2.1, after adding one free tensor multiplet to make it $\mathrm{U}(N)$. For $G=\mathrm{SO}(2 N)$, we tested it till $m^{4}, q^{1}$ order only at $N=4$, but in principle one can do all the calculus of section 2.1, following the methods of [9]. For $E_{N}$, this is just a prediction by assuming S-duality and $5 \mathrm{~d}$ perturbative results. The last term proportional to $c_{2}|G|$ will be further tested in section 3 , from the $6 \mathrm{~d}$ chiral anomaly of $\mathrm{SO}(5) \mathrm{R}$-symmetry.

\section{High temperature limit of the index}

In this section, we compute the asymptotic form of the prepotential at strong coupling, or high 'temperature' $\tau \rightarrow i 0$. This is the limit in which the compactification radius $R^{\prime}$ of the sixth circle becomes large, or equivalently in which D0-branes become light. The key technique of computation will be the anomalous S-duality that we developed in section 2.

Our convention is that the strong coupling theory of our interest is the 'S-dualized' theory. So we take $\tau_{D} \rightarrow i 0^{+}$, and $\tau=-\frac{1}{\tau_{D}} \rightarrow i \infty$. Recall $f_{\mathrm{S} \text {-dual }}$ satisfies

$$
\tau^{2} f_{\mathrm{S} \text {-dual }}\left(\tau_{D}, v_{D}, m_{D}\right)=f_{\mathrm{S} \text {-dual }}(\tau, v, m)+\frac{1}{4 \pi i \tau}\left(\frac{\partial f}{\partial v}\right)^{2}
$$

where $\tau_{D}=-\frac{1}{\tau}, v_{D}=v+\frac{1}{2 \pi i \tau} \frac{\partial f}{\partial v}, m_{D}=\frac{m}{\tau}$. We replaced $f_{\text {S-dual }}$ by $f$ when it appears with $v$ derivatives, since $f_{\text {anom }}$ is independent of $v$. Inserting $f_{\mathrm{S} \text {-dual }}=f-f_{\text {anom }}$, one finds that

$$
\tau^{2} f\left(\tau_{D}, v_{D}, m_{D}\right)=f(\tau, v, m)+\frac{1}{4 \pi i \tau}\left(\frac{\partial f}{\partial v}\right)^{2}+\tau^{2} f_{\text {anom }}\left(\tau_{D}, m_{D}\right)-f_{\text {anom }}(\tau, m) .
$$


Using $(2.90)$ and $E_{2}(-1 / \tau)=\tau^{2}\left(E_{2}(\tau)+\frac{6}{\pi i \tau}\right)$, one obtains

$$
\tau^{2} f_{\text {anom }}\left(\tau_{D}, m_{D}\right)-f_{\text {anom }}(\tau, m)=\frac{N m^{2}}{2}\left(\log (-i)-\frac{\pi i \tau_{D}}{6}+\frac{\pi i \tau}{6}\right)+\frac{N^{3} m^{4}}{48 \pi i \tau} .
$$

Inserting this in (3.2), one obtains

$$
f\left(\tau_{D}, v_{D}, m_{D}\right)=\tau^{-2} f\left(\tau, v, \tau m_{D}\right)+\frac{1}{4 \pi i \tau^{3}}\left(\frac{\partial f}{\partial v}\right)^{2}+\frac{N m_{D}^{2}}{2}\left(\log (-i)+\frac{\pi i}{6 \tau}+\frac{\pi i \tau}{6}\right)+\frac{N^{3} m_{D}^{4}}{48 \pi i} \tau .
$$

We shall study it in the limit $\tau \rightarrow i \infty$ with $m_{D}$ and $v_{D}$ fixed.

The limit $\tau \rightarrow i \infty$ on the right hand side has to be understood with care, since $m=\tau m_{D}$ scales with $\tau$. Also, we should study how $v$ scales with $\tau \rightarrow i \infty$, at fixed $v_{D}$. Had $v, m$ not scaled with $\tau$, one would have naively expected that the instanton corrections in $f$ would have been suppressed at $q \ll 1$, so that we could replace $f$ on the right hand side by $f_{\text {pert }}$. Let us check when this is correct. This expectation is correct if $F_{k}(v, m)$ does not scale to be larger than $q^{k}$. From $(2.8), F_{k}$ scales like $F_{k} \sim e^{ \pm k N m}$ at $\pm \operatorname{Re}(m) \gg 1$. For this factor to be smaller than $q^{k}$, one should require $\left|\operatorname{Re}\left(\tau m_{D}\right)\right|<-\frac{2 \pi i \tau}{N}$. Let us take $\tau$ to be purely imaginary for convenience (although most of our final results are valid for complex $\tau$ ). Then, $F_{k}$ can be ignored if

$$
\left|\operatorname{Im}\left(m_{D}\right)\right|<\frac{2 \pi}{N} .
$$

When $\operatorname{Im}\left(m_{D}\right)$ reaches $\pm \frac{2 \pi}{N}$, we encounter a phase transition, beyond which one should make a new $q$ expansion on the right hand side. The correct nature of this phase transition will be commented on later. To make the simplest calculus at $\tau_{D} \rightarrow i 0^{+}$, we take $m_{D}$ to satisfy (3.5).

Let us also discuss how $v$ should scale at fixed $v_{D}$. We shall first assume that $v$ is finite at finite $v_{D}$, and then show that it is consistent with ignoring $f_{\text {inst }}$. If $f_{\text {inst }}$ can be ignored, then the relation between $v$ and $v_{D}$ can be simplified as

$$
v=v_{D}-\frac{1}{2 \pi i \tau} \frac{\partial f_{\text {pert }}}{\partial v}\left(v, \tau m_{D}\right)
$$

$\frac{\partial f_{\text {pert }}}{\partial v}$ is given by

$$
\frac{\partial f_{\text {pert }}}{\partial v}=\sum_{\alpha \in \Delta} \alpha\left[\operatorname{Li}_{2}\left(e^{\alpha \cdot v}\right)-\frac{1}{2} \operatorname{Li}_{2}\left(e^{\alpha \cdot v \pm m}\right)\right] .
$$

Since we assume that $v$ is finite, the first term not containing $m$ yields a subleading contribution, from the $\frac{1}{\tau} \rightarrow 0$ factor in (3.6). To be definite, we take $\operatorname{Im}\left(m_{D}\right)<0$ so that $\operatorname{Re}(m) \gg 1$. Then,

$$
\operatorname{Li}_{2}\left(e^{\alpha(v)+m}\right)=-\operatorname{Li}_{2}\left(e^{-\alpha(v)-m}\right)-\frac{\pi^{2}}{6}-\frac{1}{2}(\log (-1)+\alpha(v)+m)^{2}
$$

where we used $\operatorname{Li}_{2}\left(e^{x}\right)+\operatorname{Li}_{2}\left(e^{-x}\right)=-\frac{\pi^{2}}{6}-\frac{1}{2}\left(\log \left(-e^{x}\right)\right)^{2}$ with the branch cut at $e^{x} \in(1, \infty)$. So one can approximate

$$
\frac{\partial f_{\text {pert }}}{\partial v} \sim-\frac{1}{2} \sum_{\alpha \in \Delta} \alpha\left[-\frac{1}{2}(m+\alpha(v)+\log (-1))^{2}\right],
$$


where $-\operatorname{Li}_{2}\left(e^{-\alpha(v)-m}\right)$ can be ignored at $\operatorname{Re}(m) \gg 1$. We ignored all the terms that vanish after summing over $\alpha$, or are subleading in the $\frac{1}{\tau} \rightarrow 0$ limit. Expanding the square on the right hand side, the term proportional to $m^{2}=m_{D}^{2} \tau^{2}$ will vanish upon summing over $\alpha$. The next term proportional to $m \alpha(v)$ will be the nonzero leading term. One obtains

$$
v^{i} \approx v_{D}^{i}-\frac{m_{D}}{4 \pi i} \sum_{\alpha \in \Delta} \alpha^{i} \alpha \cdot v=v_{D}^{i}-\frac{N m_{D}}{2 \pi i}(P v)^{i},
$$

where we used

$$
\sum_{\alpha \in \Delta} \alpha \otimes \alpha=\sum_{i \neq j}\left(e^{i}-e^{j}\right) \otimes\left(e^{i}-e^{j}\right)=2(N-1) \mathbf{1}_{N \times N}-2 \sum_{i \neq j} e^{i} \otimes e^{j} \equiv 2 N P .
$$

Here, $P$ is the $N \times N$ projection to $\mathrm{SU}(N)$. Decomposing $v=v_{\mathrm{U}(1)}+v_{\mathrm{SU}(N)}$, one finds that

$$
v_{\mathrm{U}(1)}=\left(v_{\mathrm{U}(1)}\right)_{D}, \quad v_{\mathrm{SU}(N)} \approx \frac{1}{1+\frac{N m_{D}}{2 \pi i}}\left(v_{\mathrm{SU}(N)}\right)_{D}
$$

at $\tau \rightarrow i \infty .{ }^{3}$ Inserting this back to $\frac{\partial f_{\text {pert }}}{\partial v}$, one obtains

$$
\frac{\partial f_{\mathrm{pert}}}{\partial v^{i}} \approx-\frac{\tau m_{D}}{2} \sum_{\alpha \in \Delta} \alpha^{i} \alpha \cdot v=-N \tau m_{D}\left(v_{\mathrm{SU}(N)}\right)^{i}=-\frac{N m_{D}}{1+\frac{N m_{D}}{2 \pi i}} \tau\left(v_{\mathrm{SU}(N)}\right)_{D}^{i} .
$$

Also $f_{\text {pert }}$ itself is given by

$$
f_{\text {pert }}=\sum_{\alpha \in \mathbf{a d j}}\left(\operatorname{Li}_{3}\left(e^{\alpha(v)}\right)-\frac{1}{2} \operatorname{Li}_{3}\left(e^{\alpha(v) \pm m}\right)\right) \approx \frac{1}{12} \sum_{\alpha \in \mathbf{a d j}} m^{3}=\frac{N^{2} \tau^{3} m_{D}^{3}}{12}
$$

where we used $\operatorname{Li}_{3}\left(e^{x}\right) \approx-\frac{x^{3}}{6}-\frac{\pi i x^{2}}{2}+\frac{\pi^{2} x}{3}$ if the real part of $x$ is positive and large.

Therefore, the asymptotic prepotential is given by

$$
\begin{aligned}
f\left(\tau_{D}, v_{D}, m_{D}\right) & \rightarrow \tau^{-2} f\left(\tau, v, \tau m_{D}\right)+\frac{1}{4 \pi i \tau^{3}}\left(\frac{\partial f}{\partial v}\right)^{2}+\frac{\pi i N m_{D}^{2} \tau}{12}+\frac{N^{3} m_{D}^{4} \tau}{48 \pi i} \\
& \approx \frac{N^{2} m_{D}^{3} \tau}{12}+\frac{\pi i N m_{D}^{2} \tau}{12}+\frac{N^{3} m_{D}^{4} \tau}{48 \pi i} .
\end{aligned}
$$

In particular, one finds that the Coulomb VEV $v_{D}$ does not appear in the asymptotic limit. This is natural since the Coulomb VEV is a dimensionful parameter, which should not be visible in the large momentum limit. This is a result for $-\frac{2 \pi}{N}<\operatorname{Im}\left(m_{D}\right)<0$. When $0<\operatorname{Im}\left(m_{D}\right)<\frac{2 \pi}{N}$, all the analysis above is same except the step of approximating $\frac{1}{\tau^{2}} f_{\text {pert }}\left(v, \tau m_{D}\right)$. In this case, $\frac{N^{2} m_{D}^{3}}{12} \tau$ is replaced by $-\frac{N^{2} m_{D}^{3}}{12} \tau$. Combining the two cases, one obtains

$$
f_{\text {asymp }}^{( \pm)}=-\frac{\pi^{3} i \tau}{3 N}\left[\left(\frac{i N m_{D}}{2 \pi}\right)^{2} \pm 2\left(\frac{i N m_{D}}{2 \pi}\right)^{3}+\left(\frac{i N m_{D}}{2 \pi}\right)^{4}\right]
$$

\footnotetext{
${ }^{3}$ At $m_{D}=-\frac{2 \pi i}{N}$, one finds that $v_{\mathrm{SU}(N)}$ diverges. In this case, one has to approximate (3.6) by assuming that $v$ can scale with $\tau$. At $m_{D}=-\frac{2 \pi i}{N}$, we checked for $N=2,3$ that $v_{\mathrm{SU}(N)}$ scales like $\sqrt{\tau}$, which grows large but is much smaller than $m=\tau m_{D}$. Due to this fact, $v$ does not affect the asymptotic free energy, and our final result for $f_{\text {asymp }}$ below will be reliable even at $m_{D}=-\frac{2 \pi i}{N}$.
} 

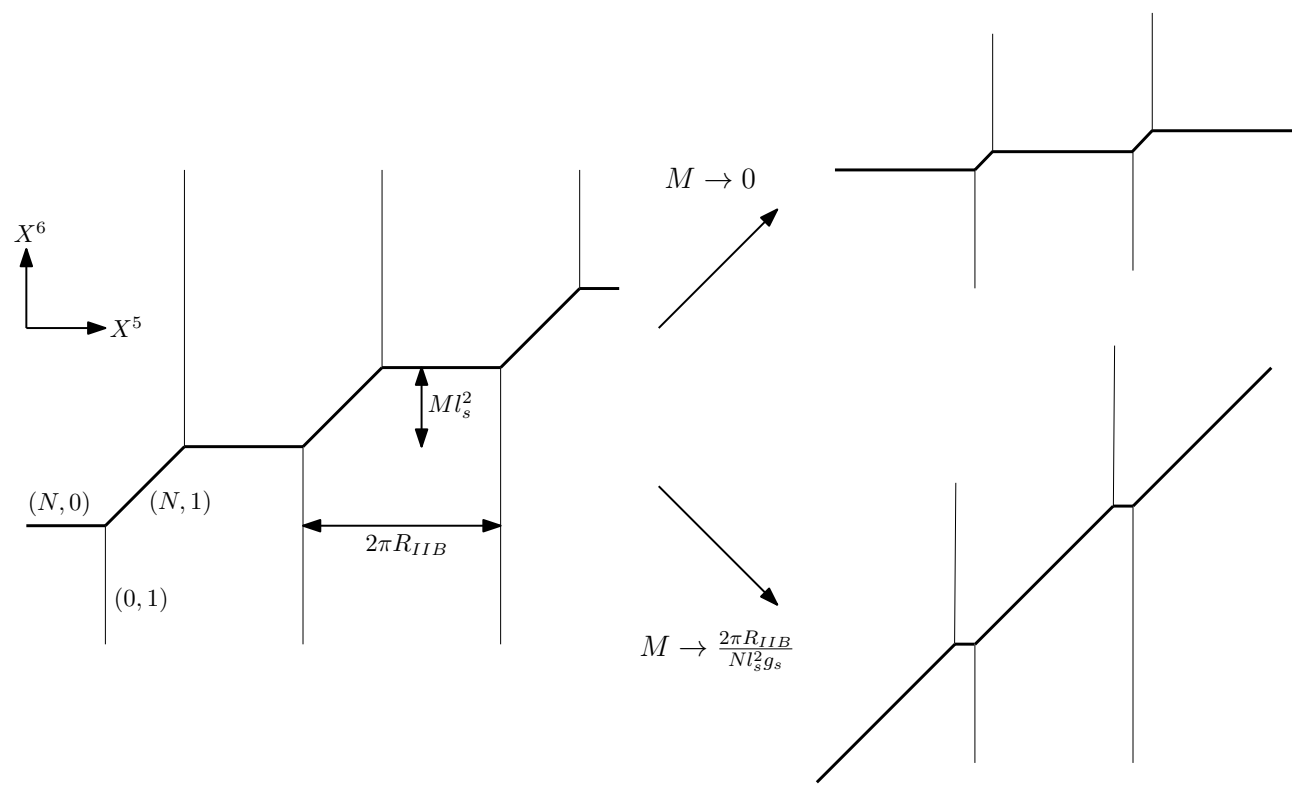

Figure 1. Type IIB 5-brane web for the $5 \mathrm{~d} \mathcal{N}=1^{*}$ system.

where \pm signs are for $0< \pm \operatorname{Im}\left(m_{D}\right)<\frac{2 \pi}{N}$, respectively. Finally, when $\operatorname{Im}\left(m_{D}\right)=0$, $\frac{1}{\tau^{2}} f_{\text {pert }}\left(v, \tau m_{D}\right)$ provides subleading contribution in $\tau$ so that one finds

$$
f_{\text {asymp }}^{(0)}=-\frac{\pi^{3} i \tau}{3 N}\left[\left(\frac{i N m_{D}}{2 \pi}\right)^{2}+\left(\frac{i N m_{D}}{2 \pi}\right)^{4}\right],
$$

where the superscript (0) means vanishing imaginary part of $m_{D}$. At this stage, we note that $f_{\text {asymp }}$ at $\operatorname{Im}\left(m_{D}\right) \neq 0$ can be written as the following holomorphic function with a branch cut,

$$
f_{\text {asymp }}=-\frac{i \tau}{2 \pi N}\left(2 \operatorname{Li}_{4}(1)-\operatorname{Li}_{4}\left(e^{N m_{D}}\right)-\operatorname{Li}_{4}\left(e^{-N m_{D}}\right)\right) .
$$

This expression will be helpful later.

We first investigate $f_{\text {asymp }}$ for purely imaginary $m_{D} \equiv i x$, at $-\frac{2 \pi}{N}<x<\frac{2 \pi}{N}$. One finds

$$
f_{\text {asymp }}=-\frac{\pi^{3} i \tau}{3 N}\left[\left(\frac{N x}{2 \pi}\right)^{2}-2\left|\frac{N x}{2 \pi}\right|^{3}+\left(\frac{N x}{2 \pi}\right)^{4}\right] .
$$

The partition function undergoes a phase transition at $x=0$, from certain perturbative particles being massless at $m_{D} \sim x=0$. One may wonder how $f_{\text {asymp }}$ behaves beyond $x= \pm \frac{2 \pi}{N}$. At $x= \pm \frac{2 \pi}{N}$, one finds from the S-dual picture that $f_{\text {inst }}$ cannot be ignored, since $F_{k} q^{k} \sim\left(e^{ \pm N m} e^{2 \pi i \tau}\right)^{k} \sim \mathcal{O}(1)$ at $m=\tau m_{D} \rightarrow \mp 2 \pi i \tau$. This means that particles with nonzero instanton number become light at these points. One can get some insights on these nonperturbative massless particles.

To see this, it is helpful to recall the type IIB 5 -brane web realization of the $5 \mathrm{~d} \mathcal{N}=1^{*}$ system. More precisely, we realize the 'S-dualized' setting at $\tau \rightarrow i \infty$, using weakly coupled type IIB string theory. The brane web first consists of $N$ D5-branes and 1 NS5-brane, all 
extended on 01234 directions, transverse to 789 , and forming a web on the $x^{5}-x^{6}$ plane. One makes a twisted compactification $\left(x^{5}, x^{6}\right) \sim\left(x^{5}+2 \pi R_{\mathrm{IIB}}, x^{6}+M \ell_{s}^{2}\right)$. The D5-branes wrap $x^{5}$ direction, and form a web with the NS5-brane extended along $x^{6}$, as shown in figure 1 . The twisted compactification guarantees that the open strings with tension $\tau_{\mathrm{F} 1}=\frac{1}{2 \pi \alpha^{\prime}}$ (where $\alpha^{\prime}=\ell_{s}^{2}$ ) suspended between D5-branes across the web have mass $\frac{M}{2 \pi}$. D1-branes wrapping $x^{5}$ ending on NS5-brane are identified as Yang-Mills instantons. Unit instanton's mass is given by $\tau_{\mathrm{D} 1} \cdot 2 \pi R_{\mathrm{IIB}}=\frac{2 \pi R_{\mathrm{II}}}{2 \pi \alpha^{\prime} g_{s}}$, which should be identified with $\frac{1}{R^{\prime}}$ in our M5-brane setting. So one obtains $2 \pi R_{\mathrm{IIB}}=\frac{2 \pi \alpha^{\prime} g_{s}}{R^{\prime}}$. On the other hand, $\tau$ is given at zero axion by $\tau=\frac{i}{g_{s}}$, which should be identified in our M5-brane setting as $i \frac{R}{R^{\prime}}$. So one finds $g_{s}=\frac{R^{\prime}}{R}$. These provide the relations between the parameters $R_{\mathrm{IIB}}, g_{s}$ and the M5-brane parameters $R, R^{\prime}$. The slope of the $(N, 1) 5$-brane is $\frac{\Delta x^{6}}{\Delta x^{5}}=\frac{1}{g_{s} N}$, where $\Delta x^{5}$ and $\Delta x^{6}$ are the distances between the two ends of the segment on figure 1 . We stated above that $\Delta x^{6}=M \alpha^{\prime}$, so one finds $\Delta x^{5}=N M g_{s} \alpha^{\prime}$.

In this setting, the segment of $(N, 1) 5$-brane shrinks at $M=0$. Here, the perturbative hypermultiplet particle becomes massless, corresponding to the fundamental strings connecting D5-branes across the NS5-brane. This causes the so-called flop phase transition. The singular term proportional to $|x|^{3}$ in (3.19) is caused by $f_{\text {pert }}$ in the S-dual setting, from the particles becoming massless at $M=0$. So we conclude that the cusp $\propto|x|^{3}$ is due to the flop transition.

As one increases positive $M$, the next transition happens when the $(N, 1)$ brane segment goes around the circle in $x^{5}$ direction, as shown on the bottom-right side of figure 1 . This happens at $\Delta x^{5}=N M g_{s} \alpha^{\prime}=2 \pi R_{\mathrm{IIB}}$,

$$
N M g_{s} \alpha^{\prime}=2 \pi R_{\mathrm{IIB}}=\frac{2 \pi \alpha^{\prime} g_{s}}{R^{\prime}}=\frac{2 \pi \alpha^{\prime}}{R} .
$$

So one finds that the transition happens at

$$
m_{D}=\frac{m}{\tau}=\frac{M R}{i / g_{s}}=-\frac{2 \pi i}{N},
$$

precisely when $F_{\text {inst }}$ cannot be ignored. Across $x= \pm \frac{2 \pi}{N}$, i.e. $m=\mp \frac{2 \pi i \tau}{N}$, the $N$ D5-brane segment shrinks. So across this value, another transition happens, with the D1-brane segment extended along the shrinking segment being massless.

As one continues to change $M$, transitions due to non-perturbative massless particles will happen at $x=\frac{2 \pi n}{N}$ with $n$ being integers. At $n=1,2, \cdots, N-1$, the nature of this transition is hard to study. This is because the massless particles are nontrivial bound states of D1-branes. Also, studying the $\tau \rightarrow i \infty$ approximations around $x=0$, not all massless particles were responsible for the cusp at $x=0$. So it will be important to know which types of massless particles contribute to the cusp of $f_{\text {asymp }}$ at $x=\frac{2 \pi n}{N}$. However, if $n$ is a multiple of $N$, one finds from the 5-brane web diagram that the transition is an $\mathrm{SL}(2, \mathbb{Z})$ transformation of the transition at $x=0$, so that the same type of cusp will happen. Indeed this has to be the case, since $x \sim x+2 \pi$ (or $m_{D} \sim m_{D}+2 \pi i$ ) is the periodicity of the instanton partition function.

Interestingly, if one takes the holomorphic extension (3.18) within $\left|\operatorname{Im}\left(m_{D}\right)\right|<\frac{2 \pi}{N}$ to the whole region of $m_{D}$, one gets a definite prediction on $f_{\text {asymp }}$ as a function of real $x$, 


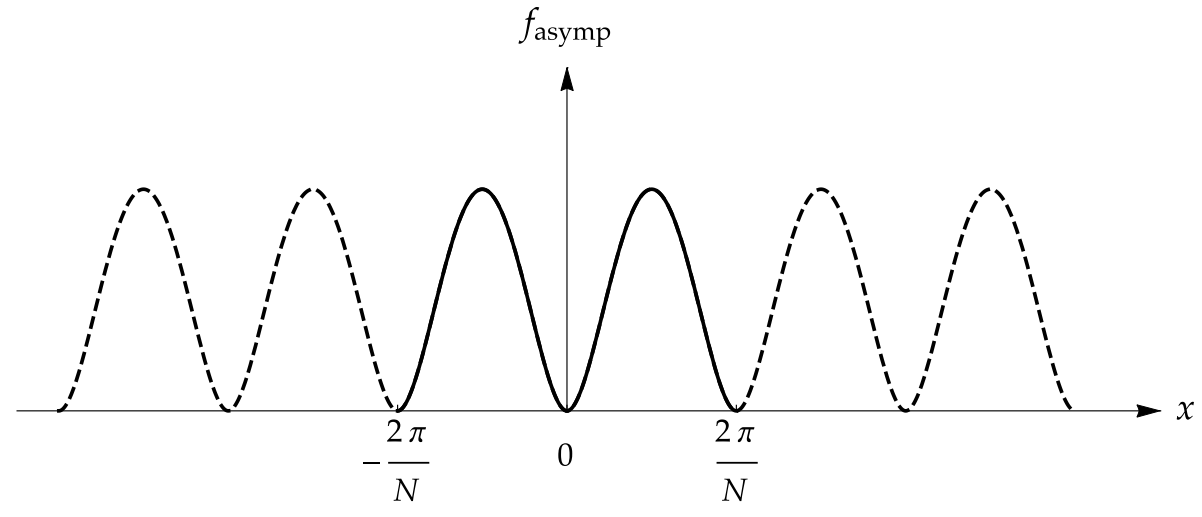

Figure 2. Continuation of the asymptotic free energy across flop transitions.

and also on the nature of phase transitions at all $n$. Plotting (3.18) for the entire real $x$, one finds figure 2. $f_{\text {asymp }}(x)$ for $\frac{2 \pi n}{N}<x<\frac{2 \pi(n+1)}{N}$ is given by simply translating the function in the range $0<x<\frac{2 \pi}{N}$ by $\frac{2 \pi n}{N}$. This means that all the cusp structures are completely the same at all $n$, at least in $f_{\text {asymp }}(x)$. It will be interesting to understand how the non-perturbative massless particles cause the same cusp in (3.18). Also, in (3.18) or in figure $2, f_{\text {asymp }}$ has a shorter period $x \sim x+\frac{2 \pi}{N}$. It will be interesting to see if the reduced period has to do with multiple-wrapping of M5-branes on $S^{1}$, analogous to the mutiple-winding fundamental strings [28].

Now we study $f_{\text {asymp }}$ for purely real $m_{D}$. The asymptotic free energy is given by

$$
-\log Z \sim \frac{f_{\text {asymp }}^{(0)}}{\epsilon_{1} \epsilon_{2}}=\frac{i}{3 \epsilon_{1} \epsilon_{2} \tau_{D}}\left[\frac{N^{3} m_{D}^{4}}{16 \pi}-\frac{\pi N m_{D}^{2}}{4}\right] .
$$

Holding real $m_{D}$ fixed, and further taking the large $N$ limit, one finds that the free energy is proportional to $N^{3}$. Namely, one finds that the single particle index $f\left(\tau, \epsilon_{1}, \epsilon_{2}, m, v\right)$ in the limit $\epsilon_{1,2} \rightarrow 0, \tau \rightarrow i 0^{+}$is given by

$$
\sum_{n=1}^{\infty} \frac{1}{n} f\left(n \tau, \epsilon_{1,2}=0, n m, n v\right) \rightarrow-\frac{i}{3 \epsilon_{1} \epsilon_{2} \tau}\left[\frac{N^{3} m^{4}}{16 \pi}-\frac{\pi N m^{2}}{4}\right],
$$

where we dropped the $D$ subscripts. This shows that the microscopic entropy (with minus sign for fermions) of light D0-branes bound to $N$ D4-branes exhibit large number of bound states proportional to $N^{3}$. The second term proportional to $N$ clearly comes from $N$ free tensor multiplets, as this comes from the S-dualization of $N f_{\mathrm{U}(1)}$. One can understand that the first term proportional to $N^{3} m^{4}$ is a remnant of the cancelation between bosonic and fermionic states in the index, since this term vanishes at $m=0$. It will be interesting to guess what kind of index $f\left(\tau, \epsilon_{1,2}=0, m, v\right)$ would exhibit the above behavior in the high temperature limit. In particular, having the analytic expression (3.18) given in terms of $\mathrm{Li}_{4}$ functions, with chemical potentials multiplied by $N$, it will be interesting to seek for an interpretation using multiple-wrapping of M5-branes, or instanton partons [29].

Finally, we comment that one can obtain the asymptotic free energy at $\tau_{D} \rightarrow i 0^{+}$for all ADE theories, starting from (2.135) and following the analysis of this section. To make 
a similar calculation, one also needs to know the perturbative partition function, and the range of $\operatorname{Im}\left(m_{D}\right)$ in which the instanton correction $f_{\text {inst }}$ can be ignored on the right hand side. The perturbative prepotential is straightforward for all ADE. As for the instanton part, we should know when $F_{k} q^{k}$ is much smaller than 1 at $q \rightarrow 0$ for large real part of $m=\tau m_{D}$. The leading behavior of $F_{k}$ for large real $m$ can be easily inferred, by knowing the correct parameter scalings between the $5 \mathrm{~d} \mathcal{N}=1^{*}$ theory and the pure $\mathcal{N}=1$ theory. Namely, one finds

$$
F_{k} q^{k} \sim e^{k c_{2} m} q^{k}
$$

where $c_{2}$ is the dual Coxeter number of the gauge group $G$. This is because the pure $5 \mathrm{~d}$ $\mathcal{N}=1$ theory is obtained by taking the limit $m \rightarrow \infty, q \rightarrow 0$, with $\Lambda \sim e^{c_{2} m} q$ held fixed. This means that one can ignore the instanton part in the region $-\frac{2 \pi}{c_{2}}<\operatorname{Im}\left(m_{D}\right)<\frac{2 \pi}{c_{2}}$. By following the analysis for the $\mathrm{U}(N)$ case, the asymptotic free energies of ADE theories are given by

$$
f_{\text {asymp }}^{( \pm)}=-\frac{\pi^{3} i \tau}{3}\left[r\left(\frac{i m_{D}}{2 \pi}\right)^{2} \pm 2|G|\left(\frac{i m_{D}}{2 \pi}\right)^{3}+\left(c_{2}|G|+r\right)\left(\frac{i m_{D}}{2 \pi}\right)^{4}\right],
$$

where \pm signs are for $0< \pm \operatorname{Im}\left(m_{D}\right)<\frac{2 \pi}{c_{2}}$, and

$$
f_{\text {asymp }}^{(0)}=-\frac{\pi^{3} i \tau}{3}\left[r\left(\frac{i m_{D}}{2 \pi}\right)^{2}+\left(c_{2}|G|+r\right)\left(\frac{i m_{D}}{2 \pi}\right)^{4}\right]
$$

for $\operatorname{Im}\left(m_{D}\right)=0$.

\subsection{Tests with U(1) partition function}

We provide a small consistency check of $f_{\text {asymp }}$ for the $\mathrm{U}(1)$ case. By this exercise, one can also get better intuitions on the true nature of the approximations and phase transitions, which perhaps may be a bit obscure in our S-duality based approach.

In the previous S-duality based approach, we first took $\epsilon_{1}, \epsilon_{2} \rightarrow 0$ limit of the partition function, to focus on the prepotential only. Then using the S-duality, we extracted out the $\beta \rightarrow 0$ asymptotics of the prepotential, where $q=e^{2 \pi i \tau}=e^{-\beta}$, at finite $m$ and $N$. We reconsider the same limits directly with the $\mathrm{U}(1)$ instanton partition function. The instanton partition function is given by

$$
Z_{\text {inst }}=\exp \left[\sum_{n=1}^{\infty} \frac{1}{n} \frac{\sinh \frac{n\left(m \pm \epsilon_{-}\right)}{2}}{\sinh \frac{n \epsilon_{1}}{2} \sinh \frac{n \epsilon_{2}}{2}} \frac{e^{-n \beta}}{1-e^{-n \beta}}\right] \sim \exp \left[\frac{4}{\epsilon_{1} \epsilon_{2}} \sum_{n=1}^{\infty} \frac{\sinh ^{2} \frac{n m}{2}}{n^{3}} \frac{e^{-n \beta}}{1-e^{-n \beta}}\right]
$$

in the $\epsilon_{1}, \epsilon_{2} \rightarrow 0$ limit. Now we take the $\beta \rightarrow 0$ limit at fixed $m$. This is somewhat tricky at real $m$, which we also take to be positive. This is because the above formula is valid for $m<\beta$ when $m$ is real. Physically, this is because the partition function $Z$ has poles at $m=n \beta$ for all positive integers $n$. So with fixed real $m$, one would hit many poles as one takes the $\beta \rightarrow 0$ limit. To deal with this situation more easily, we first continue $m$ to be purely imaginary, $m=i x$, and continue back later to complex $m$. 
Inserting $m=i x$ and taking $\beta \rightarrow 0$ limit, one obtains

$$
\begin{aligned}
Z & \sim \exp \left[-\frac{4}{\epsilon_{1} \epsilon_{2} \beta} \sum_{n=1}^{\infty} \frac{\sin ^{2} \frac{n x}{2}}{n^{4}}\right]=\exp \left[\frac{1}{\epsilon_{1} \epsilon_{2} \beta} \sum_{n=1}^{\infty} \frac{1}{n^{4}}\left(e^{i n x}+e^{-i n x}-2\right)\right] \\
& =\exp \left[\frac{1}{\epsilon_{1} \epsilon_{2} \beta}\left(\operatorname{Li}_{4}\left(e^{i x}\right)+\operatorname{Li}_{4}\left(e^{-i x}\right)-2 \operatorname{Li}_{4}(1)\right)\right]
\end{aligned}
$$

The final expression can be continued to complex $x$. Here, we use the property

$$
\operatorname{Li}_{n}\left(e^{2 \pi i x}\right)+(-1)^{n} \operatorname{Li}_{n}\left(e^{-2 \pi i x}\right)=-\frac{(2 \pi i)^{n}}{n !} B_{n}(x),
$$

where $0 \leq \operatorname{Re}(x)<1$ for $\operatorname{Im}(x) \geq 0 . B_{n}(x)$ are the Bernoulli polynomials, given by

$$
\frac{t e^{x t}}{e^{t}-1}=\sum_{n=0}^{\infty} B_{n}(x) \frac{t^{n}}{n !} \text {. }
$$

In particular, one finds $B_{4}(x)=\frac{1}{30}\left(-1+30 x^{2}-60 x^{3}+30 x^{4}\right)$, so that

$$
\mathrm{Li}_{4}\left(e^{i x}\right)+\mathrm{Li}_{4}\left(e^{-i x}\right)=-\frac{(2 \pi)^{4}}{24} B_{4}(x / 2 \pi)=\frac{2 \pi^{4}}{90}-\frac{2 \pi^{4}}{3}\left[\left(\frac{x}{2 \pi}\right)^{2}-2\left(\frac{x}{2 \pi}\right)^{3}+\left(\frac{x}{2 \pi}\right)^{4}\right]
$$

for $0 \leq x<2 \pi$. This leads to the asymptotic formula

$$
-\log Z \sim \frac{2 \pi^{4}}{3 \epsilon_{1} \epsilon_{2} \beta}\left[\left(\frac{x}{2 \pi}\right)^{2}-2\left(\frac{x}{2 \pi}\right)^{3}+\left(\frac{x}{2 \pi}\right)^{4}\right]
$$

for $0 \leq x<2 \pi$, which is in complete agreement with the S-duality-based result, (3.19), upon inserting $-i \tau \rightarrow \frac{2 \pi}{\beta}$ and $N=1$. When $-2 \pi<x \leq 0$, we use a different identity of $\mathrm{Li}_{4}$ function to find a similar expression, with the sign of the $\mathcal{O}\left(x^{3}\right)$ term flipped. This also shows that the continuation (3.18) beyond $-2 \pi<x<2 \pi$ by the $\mathrm{Li}_{4}$ functions to complex $x$ is indeed correct.

\section{$3.26 \mathrm{~d}$ chiral anomalies on Omega-deformed $\mathbb{R}^{4} \times T^{2}$}

In this subsection, we shall discuss the connection between the S-duality anomaly and the $6 \mathrm{~d}$ chiral anomalies of global symmetries. In particular, we shall independently compute some part of our asymptotic free energy $f_{\text {asymp }}$ based on chiral anomaly only. However, let us start by giving a general comment, on why one should naturally expect S-duality anomaly of the partition function if the system has chiral anomaly.

Consider a partition function of even dimensional chiral theories on $T^{2}$, like $2 \mathrm{~d}$ theories on $T^{2}$ or our system on $\mathbb{R}^{4} \times T^{2}$. For a global symmetry, one turns on a background gauge field $A$. In particular, let us turn on the flat connection of $A$ on $T^{2}$. We shall only be interested in Abelian flat connections, characterized by the commuting holonomies along the two circles of $T^{2}$. Large gauge transformations would have made both holonomies to be periodic, had there been no chiral anomalies. For simplicity, let us take a rectangular torus with two radii $r_{1}, r_{2}$, respectively. Then the large gauge transformations would have given the periods $A_{1} \sim A_{1}+\frac{1}{r_{1}}$ and $A_{2} \sim A_{2}+\frac{1}{r_{2}}$. With matter fields having 
integral charge $q$ of this global symmetry, the modes of these fields would have frequencies $\left(\omega_{1}, \omega_{2}\right)=\left(\frac{n_{1}}{r_{1}}+q A_{1}, \frac{n_{2}}{r_{2}}+q A_{2}\right)$ on $T^{2}$, with integral $n_{1}, n_{2}$, which is invariant under the periodic shifts of $A_{1}, A_{2}$. This is a consequence of these gauge symmetries. However, in quantum observables like the partition function on $T^{2}$, these large gauge transformations may fail to be symmetries for theories with chiral anomalies. This is because one has to regularize the path integral over these modes, by regarding one of the two directions as temporal circle [30]. By this procedure, one of the two holonomies $A_{1}, A_{2}$ fail to be periodic in the partition functions. This is precisely what happen for the $2 \mathrm{~d}$ elliptic genera [31]. We expect that similar things will happen to $6 \mathrm{~d}$ chiral theories on $\mathbb{R}^{4} \times T^{2}$, but we cannot make this expectation more precise here. We shall simply assume the failure of double periodicity of background holonomies due to chiral anomalies, and then explain that it forces the partition function to have S-duality anomaly, as we found in section 2 by nonzero $F_{\text {anom }}$.

Let us write the background holonomies as a complex number $m$. Had a free energy $F(\tau, m)$ on $T^{2}$ been exactly S-dual, then its exact S-duality $F(-1 / \tau, m / \tau)=F(\tau, m)$ means that $m$ has double period. This is because if the right hand side has period in one direction, say $F(\tau, m)=F(\tau, m+1)$, the left hand side forces $F\left(-\frac{1}{\tau}, \frac{m}{\tau}\right)=F\left(-\frac{1}{\tau}, \frac{m}{\tau}+\frac{1}{\tau}\right)$, and thus $F(\tau, m)=F(\tau, m-\tau)$, contradicting the obstruction of double periodicity from chiral anomaly. This comment applies to our $6 \mathrm{~d}$ partition functions. So we naturally expect S-duality anomaly.

With these motivations in mind, rather than trying to elaborate on it, we shall make a concretely calculation which shows that a particular term in our asymptotic high temperature free energy dictated by $F_{\text {anom }}$ can be computed using $6 \mathrm{~d}$ chiral anomaly only.

Let us first explain the anomalies of the $6 \mathrm{~d}(2,0)$ theory of $A_{N-1}$ type. More precisely, we shall consider the anomaly of the interacting $A_{N-1}$ type theory times a decoupled free self-dual tensor multiplet theory. This corresponds to the system of $N$ M5-branes including the decoupled center-of-mass multiplet. The anomaly polynomial 8 -form is given by

$$
I_{8}=N I_{8}(1)+N\left(N^{2}-1\right) \frac{p_{2}(N)}{24}
$$

where $I_{8}(1)$ is the anomaly of the single M5-brane theory, or one free $(2,0)$ tensor multiplet,

$$
I_{8}(1)=\frac{1}{48}\left[p_{2}(N)-p_{2}(T)+\frac{1}{4}\left(p_{1}(T)-p_{1}(N)\right)^{2}\right] .
$$

The Pontryagin classes are defined by

$$
p_{1}=-\frac{1}{2(2 \pi)^{2}} \operatorname{tr} R^{2}, \quad p_{2}=\frac{1}{(2 \pi)^{4}}\left[-\frac{1}{4} \operatorname{tr} R^{4}+\frac{1}{8}\left(\operatorname{tr} R^{2}\right)^{2}\right] .
$$

Here, traces are acting on either $6 \times 6$ matrices for $\mathrm{SO}(5,1)$ tangent bundle $T$, or $5 \times 5$ matrices for $\mathrm{SO}(5)$ normal bundle $N$. Taking their curvatures to be $R$ and $F$, respectively, one finds

$$
\begin{aligned}
(2 \pi)^{4} I_{8}= & \frac{N}{48}\left[-\frac{1}{4} \operatorname{tr} F^{4}+\frac{1}{8}\left(\operatorname{tr} F^{2}\right)^{2}+\frac{1}{4} \operatorname{tr} R^{4}-\frac{1}{8}\left(\operatorname{tr} R^{2}\right)^{2}+\frac{1}{16}\left(\operatorname{tr} R^{2}-\operatorname{tr} F^{2}\right)^{2}\right] \\
& +\frac{N^{3}-N}{24}\left(-\frac{1}{4} \operatorname{tr} F^{4}+\frac{1}{8}\left(\operatorname{tr} F^{2}\right)^{2}\right) .
\end{aligned}
$$


We shall restrict $F$ to a Cartan part. In particular, since we shall be taking the Omega backgrounds to be small, the Cartan for $\mathrm{SU}(2)_{R}$ will have much smaller background field than $\mathrm{SU}(2)_{L}$, from $\epsilon_{+} \ll m$. So we shall only turn on the background field for the Cartan in $\mathrm{SU}(2)_{L} \subset \mathrm{SO}(5)$, corresponding to our $\mathcal{N}=1^{*}$ mass $m$. $F$ is a $5 \times 5$ matrix-valued 2-form, whose components are $F^{a b}=-F^{b a}$ with $a, b=1, \cdots, 5$. The component corresponding to the Cartan of $\mathrm{SU}(2)_{L}$ is obtained by keeping $F^{12}=-F^{21}=-F^{34}=F^{43} \equiv F$ only. With this restriction, one finds $\operatorname{tr}\left(F^{2}\right) \rightarrow-4 F^{2}, \operatorname{tr}\left(F^{4}\right) \rightarrow 4 F^{4}$. Inserting these, the $\mathrm{SO}(5,1)$ and $\mathrm{U}(1) \subset \mathrm{SU}(2)_{L}$ anomalies are given by

$$
(2 \pi)^{4} I_{8} \rightarrow \frac{N^{3}}{24} F^{4}+\frac{N}{48}\left[\frac{1}{2} F^{2} \operatorname{tr} R^{2}+\frac{1}{4} \operatorname{tr} R^{4}-\frac{1}{8}\left(\operatorname{tr} R^{2}\right)^{2}\right] .
$$

Only the first term $\frac{N^{3}}{24} F^{4}$ will be relevant for the computations below.

Our goal is to compute some part of the asymptotic free energy at high temperature $\tau_{D} \rightarrow 0$, using $6 \mathrm{~d}$ chiral anomalies. Recall that we found

$$
S_{\mathrm{eff}}=-\log Z \rightarrow \frac{f_{\mathrm{asympt}}}{\epsilon_{1} \epsilon_{2}}=\frac{i}{2^{4} \cdot 3 \pi \epsilon_{1} \epsilon_{2} \tau_{D}}\left[N^{3} m^{4}-4 \pi^{2} N m^{2}+\cdots\right]
$$

where $\cdots$ stands for the $m^{3}$ term which exists when $m$ has imaginary component. The $m^{3}$ term will not be of our interest in this subsection. We obtained this expression at $\epsilon_{1,2} \ll 1$ and $\tau_{D} \rightarrow 0$, where $\tau_{D} \equiv \frac{\beta}{4 \pi}(\mu+i)$ is the same $\tau_{D}$ used before. Often, we used purely imaginary $\tau_{D}$ with $\mu=i$, but we keep real $\mu$ in this subsection to see a clear relation to chiral anomalies. For a reason to be explained below, we would like to study the asymptotic free energy when all the chemical potentials $\epsilon_{1,2}, m$ are purely imaginary. So inserting $i \epsilon_{1,2}, i m$ (with real $\epsilon_{1,2}, m$ ) in the places of $\epsilon_{1,2}, m$ in (3.38), one obtains $S_{\text {eff }}=-\frac{i}{2^{4} \cdot 3 \pi \epsilon_{1} \epsilon_{2} \tau_{D}}\left[N^{3} m^{4}+4 \pi^{2} N m^{2}+\mathcal{O}\left(m^{3}\right)\right]$. In this setting, we focus on the imaginary part of the effective action,

$$
\operatorname{Im}\left(S_{\text {eff }}\right)=-\frac{\mu}{12 \epsilon_{1} \epsilon_{2} \beta\left(1+\mu^{2}\right)}\left[N^{3} m^{4}+4 \pi^{2} N m^{2}+\mathcal{O}\left(m^{3}\right)\right]
$$

and compute it from $6 \mathrm{~d}$ chiral anomalies. Especially, we shall compute part of $\operatorname{Im}\left(S_{\text {eff }}\right)$ from the $5 \mathrm{~d}$ effective action approach for the $6 \mathrm{~d}$ theory on small temporal circle. $6 \mathrm{~d}$ chiral anomaly determines a special class of terms in the $5 \mathrm{~d}$ effective action. It turns out that, knowing the terms determined by anomaly, one can only compute the term proportional to $m^{4}$. So we shall pay attention to the first term

$$
\left.\operatorname{Im}\left(S_{\text {eff }}\right)\right|_{m^{4}}=-\frac{\mu N^{3} m^{4}}{12 \epsilon_{1} \epsilon_{2} \beta\left(1+\mu^{2}\right)} .
$$

We shall argue below that this term is completely dictated by $6 \mathrm{~d}$ chiral anomaly, and then we re-compute this term using chiral anomaly only. This will provide another strong test of our findings from the D0-D4 calculus. Then, since one naturally expects that supersymmetrization of (3.40) is holomorphic in $\tau_{D}$, one can reconstruct the term $-\frac{i N^{3} m^{4}}{2^{4} \cdot 3 \pi \epsilon_{1} \epsilon_{2} \tau_{D}}$ in (3.38). 
We shall consider the $6 \mathrm{~d}$ anomaly from the viewpoint of $5 \mathrm{~d}$ effective action, obtained by compactification on a small circle of circumference $\beta \ll 1$, and discuss our asymptotic free energy $f_{\text {asympt }}$ on $\mathbb{R}_{\epsilon_{1,2}}^{4} \times T^{2}$ in this setting. On $T^{2}$, regarding one circle as the temporal circle, the partition function is an index of the form

$$
Z\left(\tau, v, m, \epsilon_{1,2}\right)=\operatorname{Tr}\left[(-1)^{F} e^{-\frac{\beta}{2}(H-i \mu P)} e^{\sum_{a=1}^{2} \epsilon_{a}\left(J_{a}+J_{R}\right)} e^{2 m J_{L}} e^{-v_{i} q_{i}}\right] .
$$

Real $\epsilon_{1,2}, m$ is consistent with the conventions for the partition function presented at the beginning of section 2 . In this setting, the chemical potentials $\epsilon_{1,2}, m$ will twist the translation on the temporal circle in a way that the twisted time evolution is not unitary (simply because the factors in the trace are not unitary transformations). This would cause a complex deformation of the Euclidean action by twisting with chemical potentials. ${ }^{4}$ For a technical reason, it will be convenient to keep these twistings to preserve the reality of the action. So we replace

$$
e^{\sum_{a=1}^{2} \epsilon_{a}\left(J_{a}+J_{R}\right)} e^{2 m J_{L}} e^{-v_{i} q_{i}} \rightarrow e^{i \sum_{a=1}^{2} \epsilon_{a}\left(J_{a}+J_{R}\right)} e^{2 i m J_{L}} e^{-i v_{i} q_{i}}
$$

which will make real twists of the Euclidean action. This is equivalent to the insertions of $i \epsilon_{1,2}, i m$ around (3.40). The factor $e^{-\frac{\beta}{2} H}$ demands us to consider a $6 \mathrm{~d}$ Euclidean theory whose temporal coordinate $y$ satisfies periodicity $y \sim y+\frac{\beta}{2}$. This forms a circle of the $T^{2}$. Another circle factor is labeled by $x$, which we take to have periodicity $x \sim x+2 \pi$. Defining $\tau_{D}=\frac{\beta}{4 \pi}(\mu+i)$, one obtains

$$
e^{-\frac{\beta}{2}(H-i \mu P)} \equiv e^{2 \pi i \tau_{D} \frac{H+P}{2}} e^{-2 \pi i \bar{\tau}_{D} \frac{H-P}{2}}=e^{-2 \pi \operatorname{Im} \tau_{D} H+2 \pi i \operatorname{Re} \tau_{D} P} .
$$

So $\tau_{D}$ is the complex structure of $T^{2}$. This torus is endowed with the metric

$$
d s^{2}\left(T^{2}\right)=(d x-\mu d y)^{2}+d y^{2}
$$

and periods $(x, y) \sim(x+2 \pi, y) \sim\left(x+\frac{\beta \mu}{2}, y+\frac{\beta}{2}\right)$. Including the chemical potential $\epsilon_{a}$, the metric of $\mathbb{R}^{4} \times T^{2}$ is given by

$$
d s^{2}\left(\mathbb{R}^{4} \times T^{2}\right)=\sum_{a=1,2}\left|d z_{a}-\frac{2 i \epsilon_{a}}{\beta} z_{a} d y\right|^{2}+(d x-\mu d y)^{2}+d y^{2},
$$

where $z_{a}$ are complex coordinates of $\mathbb{C}^{2} \sim \mathbb{R}^{4}$ with charges $J_{a}\left[z_{b}\right]=\delta_{a b}$. Finally, the chemical potential $m$ is realized as the background gauge field $A=\frac{2 m}{\beta} d y$ for $\mathrm{U}(1) \subset$ $\mathrm{SU}(2)_{L}$. Also, $\frac{H-P}{2} \sim\{Q, \bar{Q}\}$, where $Q$ is a supercharge preserved by the index. So $Z$ is independent of $\bar{\tau}$.

\footnotetext{
${ }^{4}$ Strictly speaking, Lagrangian formulation is not known in $6 \mathrm{~d}$. So when we refer to a Lagrangian description, we mean a 5d Lagrangian after reducing on a small circle. See also comments in [11] concerning the conversion between twistings and background gauge fields in the presence of anomalies.
} 
Following [11] (see also [32]), we shall make a KK reduction on the small circle along $y$, for small inverse-temperature $\beta \ll 1$. To this end, one rewrites the background in the form of

$$
d s^{2}=e^{2 \phi}(d y+a)^{2}+h_{i j} d x^{i} d x^{j},
$$

where $h_{i j}$ with $i, j=1, \cdots 5$ is the 5 d metric, $e^{2 \phi}=1+\mu^{2}+\frac{4}{\beta^{2}} \sum_{a} \epsilon_{a}^{2}\left|z_{a}\right|^{2}$ is the dilaton, and

$$
a=\frac{1}{1+\mu^{2}+\frac{4 \epsilon_{a}^{2}\left|z_{a}\right|^{2}}{\beta^{2}}}\left(-\mu d x-\frac{2 \epsilon_{a}\left|z_{a}\right|^{2}}{\beta} d \phi_{a}\right)
$$

is the gravi-photon field, where $z_{a}=\left|z_{a}\right| e^{i \phi_{a}}$. The $6 \mathrm{~d}$ background gauge field $A$ for $\mathrm{U}(1) \subset$ $\mathrm{SU}(2)_{L}$ is also rewritten in the form $A=A_{6}(d y+a)+\mathcal{A}$, where $\mathcal{A}$ is the $5 \mathrm{~d}$ background gauge field and $A_{6}$ is the $5 \mathrm{~d}$ scalar. So one finds $A_{6}=\frac{2 m}{\beta}$ and $\mathcal{A}=-A_{6} a$.

If the $6 \mathrm{~d}$ theory compactified on a small circle has no $5 \mathrm{~d}$ massless modes, one can express the thermal partition function in terms of a $5 \mathrm{~d}$ local effective field theory of background fields, where the $5 \mathrm{~d}$ derivative expansion corresponds to a $\beta$ series expansion. As noted in [11], with massless modes in $5 \mathrm{~d}$, there could be nonlocal part of the effective action which is smooth in the $\beta \rightarrow 0$ limit. In our case, the non-local part comes from the $5 \mathrm{~d}$ perturbative maximal SYM. There is additional difficulty in using the derivative expansion in our setting, since some of our background fields are proportional to $\beta^{-1}$, which may spoil the orderings provided by the derivative expansion. So it appears tricky to directly employ the formalism of $[11,32]$.

However, one can study the imaginary part (3.39) of our asymptotic free energy using the $5 \mathrm{~d}$ approach. The imaginary part can be computed completely by knowing the $5 \mathrm{~d}$ Chern-Simons like terms. To explain this, note first that we have been careful to set all our background fields to be real, e.g. by setting our chemical potentials to be imaginary. With real background fields turned on, suppose that we first reduce the $6 \mathrm{~d}$ theory on a small circle to a general 5d Lorentzian spacetime. Then the 5d effective action is real, since Hermiticity is not broken in the Lorentzian theory. Now we Wick-rotate the 'time' direction in this $5 \mathrm{~d}$ setting. Since all background fields are real, the only possible step which may cause complex effective action is the Wick rotation to Euclidean 5d space. Here, note that we are seeking for an effective action of the vectors $a, \mathcal{A}, \omega$ (spin connection), tensor $h_{i j}$, and scalars $A_{6}, \phi$. To compute the imaginary part, one can focus on the local terms. This is because the nonlocal terms come from the determinant of $5 \mathrm{~d}$ maximal SYM whose fields are covariantized by real background fields, which is real. Among the local terms obtained from scalar Lagrangian density, we should seek for terms containing the tensor $\epsilon^{i j k l m}$ to obtain imaginary contribution after Wick rotation. It should be contracted with antisymmetric tensors formed by the background fields. There are many possibilities, arranged in derivative expansion. For instance, there could be complicated terms like $\sim d a \wedge d \mathcal{A} \wedge d \phi f\left(\phi, A_{6}\right)$, and so on.

Although there are many terms, let us comment that there can be gauge invariant terms and gauge non-invariant terms in the imaginary action. The latter class should exist because the $5 \mathrm{~d}$ effective action should realize $6 \mathrm{~d}$ chiral anomalies. The coefficients of the terms in the latter class are thus completely determined by known $6 \mathrm{~d}$ anomalies $[11,32]$. 
Among the gauge invariant terms, there can be action coming from gauge invariant Lagrangian density, like the term that we illustrated in the last paragraph. Finally, there may be Chern-Simons terms in which Lagrangian densities are not gauge invariant but their integrals are. So the imaginary action takes the following structure,

$$
\begin{aligned}
& S_{\mathrm{CS}}=S_{\mathrm{CS}}^{(1)}+S_{\mathrm{CS}}^{(2)}+S_{\mathrm{GI}} \\
& S_{\mathrm{CS}}^{(1)}=\frac{i \kappa_{1}}{\beta^{3}} \int a \wedge d a \wedge d a+\frac{i \kappa_{2}}{\beta} \int \mathcal{A} \wedge d \mathcal{A} \wedge d a+\frac{i \kappa_{3}}{\beta} \int a \wedge R \wedge R+i \kappa_{4} \int \mathcal{A} \wedge d \mathcal{A} \wedge d \mathcal{A}+\cdots \\
& S_{\mathrm{CS}}^{(2)}=-\frac{i D r_{1}}{96 \pi^{2}} \int\left(A_{6}^{4} a \wedge d a \wedge d a+4 A_{6}^{3} \mathcal{A} \wedge d a \wedge d a+6 A_{6}^{2} \mathcal{A} \wedge d \mathcal{A} \wedge d a+4 A_{6} \mathcal{A} \wedge d \mathcal{A} \wedge d \mathcal{A}\right)+\cdots
\end{aligned}
$$

where $r_{1}=\frac{\beta}{4 \pi}$ is the radius of the small sixth circle with circumference $\frac{\beta}{2}$. $S_{\mathrm{CS}}^{(1)}$ consists of the gauge invariant Chern-Simons terms. $S_{\mathrm{CS}}^{(2)}$ is part of the gauge non-invariant ChernSimons terms that comes from $\mathrm{U}(1) \subset \mathrm{SU}(2)_{L} \subset \mathrm{SO}(5)_{R}$ normal bundle anomaly in $6 \mathrm{~d}$, namely the first term $\sim \frac{N^{3}}{24} F^{4}$ of (3.37). Anomaly matching fixes $D=N^{3}$, as well as the relative coefficients as shown on the second line. ${ }^{5}$ The omitted terms $\cdots$ in $S_{\mathrm{CS}}^{(1)}$ are other Chern-Simons terms containing $\omega$, which we do not need here. The omitted terms in $S_{\mathrm{CS}}^{(2)}$ can all be computed from mixed anomalies and gravitational anomalies of (3.37), which we do not work out here as we shall not need them. Finally, $S_{\mathrm{GI}}$ is the action containing $\epsilon^{i j k l m}$ associated with gauge invariant Lagrangian density, e.g. $d a \wedge d a \wedge d \phi f\left(\phi, A_{6}\right), d \mathcal{A} \wedge$ $d \mathcal{A} \wedge d\left[(d a)^{i j}(d \mathcal{A})_{i j}\right] g\left(\phi, A_{6}\right)$, and so on. One point we emphasize is that $S_{\text {GI }}$ can come in infinite series of derivative expansion, while $S_{\mathrm{CS}}^{(1)}$ and $S_{\mathrm{CS}}^{(2)}$ consist of finite number of terms and can be completely classified.

The imaginary terms have rich possibilities. Here we consider the terms which are nonzero with our background, and also the leading terms in small $\epsilon_{1,2}$, proportional to $\frac{1}{\epsilon_{1} \epsilon_{2}} . \quad A_{6}=\frac{2 m}{\beta}$ is constant in our background. Also, $\mathcal{A}=-A_{6} a$ is constant times the graviphoton. Plugging in these values, one obtains

$$
\left.\left(A_{6}\right)^{n} \epsilon^{i j k l m} \text { (rank } 5 \text { antisymmetric tensor of } a, \phi, \omega, h\right) \text {. }
$$

The parenthesis consists of the fields reduced from $6 \mathrm{~d}$ metric (3.45). Note that, after plugging in constant $A_{6}$ and $\mathcal{A}=-A_{6} a$, all terms should be formally gauge invariant in the remaining fields. This is because the only possible gauge non-invariant terms $S_{\mathrm{CS}}^{(2)}$, completely dictated by anomaly, also become gauge invariant like $A_{6}^{4} a \wedge d a \wedge d a$ with constant $A_{6}$.

Now we note the fact that, in the $6 \mathrm{~d}$ metric, all $z_{a}$ coordinates of $\mathbb{R}^{4}$ are multiplied by $\epsilon_{a}$. So in the parenthesis of (3.49), the only $z^{a}$ 's not associated with $\epsilon_{a}$ are derivatives. So one makes a formal derivative expansion of this term, assigning the 'mass dimensions' $[a]=0,[\phi]=0,[h]=0,[\omega]=1$. The lowest order term comes in two derivatives, and is proportional to $a \wedge d a \wedge d a$. There can be no other gauge-invariant terms at this order. This term indeed yields the desired $\frac{1}{\epsilon_{1} \epsilon_{2}}$ scaling. Firstly, the integral $d x d^{2} z_{1} d^{2} z_{1}$ can be scaled into $\left(\frac{\beta^{2}}{\epsilon_{1} \epsilon_{2}}\right)^{2}$ times a measure depending on $\frac{\epsilon_{a} z_{a}}{\beta}$. Also, two derivatives in $a \wedge d a \wedge d a$

\footnotetext{
${ }^{5}$ Following [11], we show the form of the action with constant value of $A_{6}$, taking into account the covariant anomaly rather than the consistent anomaly. This is sufficient for our calculus of the free energy.
} 
can also be scaled with $\frac{\epsilon_{a}}{\beta}$, yielding another overall factor $\frac{\epsilon_{1} \epsilon_{2}}{\beta^{2}} . z_{a}$ in the remaining integral appear in the combination $\frac{\epsilon_{a} z_{a}}{\beta}$, including the integration variable, so is independent of $\epsilon_{a}$. So this term yields the right scaling $\sim \frac{1}{\epsilon_{1} \epsilon_{2}}$. Therefore, to compute (3.40), we only need to consider those terms that reduce to

$$
\left(A_{6}\right)^{n} a \wedge d a \wedge d a
$$

upon plugging in our background. This implies that one does not have to consider $S_{\mathrm{GI}}$ of (3.48), since they are associated with local Lagrangian density and cannot provide terms like (3.50).

So we only consider $S_{\mathrm{CS}}^{(1)}$ and $S_{\mathrm{CS}}^{(2)}$ of (3.48). Unlike the coefficients of $S_{\mathrm{CS}}^{(2)}$, coefficients of $S_{\mathrm{CS}}^{(1)}$ cannot be determined with our limited knowledge of the $6 \mathrm{~d}$ theory. So even after restricting our interest to the imaginary part (3.39) of the effective action, we cannot compute them all due to our ignorance on these coefficients. Since the second term of $S_{\mathrm{CS}}^{(1)}$ is quadratic in $\mathcal{A}$, we cannot compute the $\mathcal{O}\left(\mathrm{m}^{2}\right)$ term of (3.39). This is why we shall not need the mixed anomaly contributions in $S_{\mathrm{CS}}^{(2)}$ coming from the term $\sim F^{2} \operatorname{tr} R^{2}$ in (3.37), which will also yield a contribution at $\mathcal{O}\left(\mathrm{m}^{2}\right)$, since knowing them is incomplete to compute the whole $\mathcal{O}\left(\mathrm{m}^{2}\right)$ contributions. Also, the $\mathcal{O}\left(\mathrm{m}^{3}\right)$ term cannot be computed since we do not know $\kappa_{4}$. However, the Chern-Simons terms that are quartic in $\mathcal{A}$ and $A_{6}$ are completely dictated by $6 \mathrm{~d}$ anomalies, as shown on the second line of (3.48). Note that quartic Chern-Simons term is allowed precisely because we allow gauge non-invariant Chern-Simons term, to match $6 \mathrm{~d}$ anomalies which are fourth order in the fields. Thus, we can compute (3.40) from $S_{\mathrm{CS}}^{(2)}$ of (3.48). Note also that, for imaginary chemical potentials, we have found earlier in this section that $f_{\text {asymp }}$ undergoes phase transitions due to massless particles. This only changes $\mathcal{O}\left(\mathrm{m}^{3}\right)$ or lower order terms, so that the $m^{4}$ order that we are going to compute is unaffected.

We also note in passing that, we can turn the logic around and use our D0-D4 results to constrain the $5 \mathrm{~d}$ effective action. Namely, we know from our D0-D4 calculus the $\mathcal{O}\left(\mathrm{m}^{2}\right)$ and $\mathcal{O}\left(\mathrm{m}^{3}\right)$ coefficients of $\operatorname{Im}\left(f_{\text {asymp }}\right)$, and also the vanishing of the $\mathcal{O}\left(\mathrm{m}^{0}\right)$ coefficient. This knowledge can be used to constrain $\kappa_{1}, \kappa_{2}, \kappa_{3}, \cdots$ of (3.48). This information may be useful for studying other high temperature partition functions of the $6 \mathrm{~d}(2,0)$ theories.

Coming back to the computation of (3.40), we plug $\mathcal{A}=-A_{6} a$ and $A_{6}=\frac{2 m}{\beta}$ into $S_{\mathrm{CS}}^{(2)}$ of (3.48) to obtain

$$
\frac{i N^{3}\left(A_{6}\right)^{4} r_{1}}{96 \pi^{2}} \int a \wedge d a \wedge d a .
$$

To compute this, one should evaluate the gravi-photon Chern-Simons term,

$$
\int a \wedge d a \wedge d a=\int\left(1+\mu^{2}+\frac{4 \epsilon_{a}^{2}\left|z_{a}\right|^{2}}{\beta^{2}}\right)^{-3}(-\mu d x) \wedge 2 \frac{4 \epsilon_{1} \epsilon_{2}}{\beta^{2}} 4 d x_{1} \wedge d y_{1} \wedge d x_{2} \wedge d y_{2}
$$

where $z_{a} \equiv x_{a}+i y_{a}$, with $x_{1}, y_{1}, x_{2}, y_{2}$ being the Cartesian coordinates of $\mathbb{R}^{4}$. Since $\int d x=2 \pi, \int d x_{a} d y_{a}=\pi \int d\left(r_{a}^{2}\right),(3.52)$ becomes

$$
-\frac{64 \pi^{3} \mu \epsilon_{1} \epsilon_{2}}{\beta^{2}} \int_{0}^{\infty} \frac{d\left(r_{1}^{2}\right) d\left(r_{2}^{2}\right)}{\left(1+\mu^{2}+\frac{4 \epsilon_{a}^{2} r_{a}^{2}}{\beta^{2}}\right)^{3}}=-\frac{4 \pi^{3} \mu \beta^{2}}{\epsilon_{1} \epsilon_{2}} \int_{0}^{\infty} \frac{d X d Y}{\left(1+\mu^{2}+X+Y\right)^{3}}=-\frac{2 \pi^{3} \mu \beta^{2}}{\left(1+\mu^{2}\right) \epsilon_{1} \epsilon_{2}},
$$


where $X=\frac{4 \epsilon_{1}^{2} r_{1}^{2}}{\beta^{2}}, Y=\frac{4 \epsilon_{2}^{2} r_{2}^{2}}{\beta^{2}}$. So one obtains

$$
\frac{i N^{3}\left(A_{6}\right)^{4} r_{1}}{96 \pi^{2}} \int a \wedge d a \wedge d a=-i \frac{N^{3} \beta}{3 \cdot 2^{7} \pi^{3}} \cdot \frac{16 m^{4}}{\beta^{4}} \cdot \frac{2 \pi^{3} \mu \beta^{2}}{\left(1+\mu^{2}\right) \epsilon_{1} \epsilon_{2}}=-i \frac{N^{3} m^{4} \mu}{12 \epsilon_{1} \epsilon_{2} \beta\left(1+\mu^{2}\right)},
$$

where we plugged in $r_{1}=\frac{\beta}{4 \pi}$. This precisely agrees with (3.40), based on D0-D4 calculus.

Finally, let us comment that the same calculation can be done to test some part of (3.25) for all ADE theories. For ADE, (3.25) yields the imaginary part

$$
\left.\operatorname{Im}\left(S_{\text {eff }}\right)\right|_{m^{4}}=-\frac{\mu\left(c_{2}|G|+r\right) m^{4}}{12 \epsilon_{1} \epsilon_{2} \beta\left(1+\mu^{2}\right)}
$$

simply by changing the coefficient $N^{3} \rightarrow c_{2}|G|+r$ from (3.25). On the other hand, the anomaly polynomial (3.33) is replaced by the following polynomial

$$
I_{8}=r I_{8}(1)+c_{2}|G| \frac{p_{2}(N)}{24}
$$

for ADE. Again after restricting $\mathrm{SO}(5)_{R}$ to $\mathrm{U}(1) \subset \mathrm{SU}(2)_{L}$, the term $\frac{N^{3}}{24} F^{4}$ of (3.37) is replaced by $\frac{c_{2}|G|+r}{24} F^{4}$. So the calculations of this subsection can be done by replacing all $N^{3}$ by $c_{2}|G|+r$, completely reproducing (3.55).

\section{Conclusions and remarks}

In this paper, we explored S-duality of the prepotential of the $6 \mathrm{~d}(2,0)$ theories compactified on $T^{2}$, on the Coulomb branch. We found evidences of S-duality and its anomaly. Using this result, we computed the asymptotic free energy of this system compactified on $S^{1}$ (in the index version), when the Omega background parameters $\epsilon_{1,2}$ and the chemical potential $\beta$ conjugate to the KK momentum are small. The asymptotic free energy is proportional to $N^{3}$ in a suitable large $N$ limit, showing that the light KK fields exhibit the $N^{3}$ degrees of freedom. After suitably complexifying the chemical potentials, we showed that the imaginary part of the free energy proportional to $N^{3}$ is completely reproduced from the $6 \mathrm{~d}$ chiral anomaly of the SO(5) R-symmetry. Most results are generalized to the ADE class of $(2,0)$ theories.

In the literatures, the $N^{3}$ scalings of various observables of $6 \mathrm{~d}(2,0)$ theory have been found, using various approaches. Thermal entropy of black M5-branes [33] or various other quantities are computed from the gravity dual. Chiral anomalies are computed from the anomaly inflow mechanism [34]. The supersymmetric Casimir energy on $S^{5}$ was computed from the superconformal index [35-40]. Perhaps among these, the mysteries of $6 \mathrm{~d}$ CFT may be most directly addressed from the thermal partition function calculus of [33]. So it would be desirable to have a microscopic view of this phenomenon by directly counting states of the 6d CFTs. As far as we are aware of, such a direct account for $N^{3}$ scaling of states has not been available from a microscopic quantum calculus yet. Our studies show the $N^{3}$ scalings of the microscopically counted degrees of freedom. More precisely, we compactified the $6 \mathrm{~d}$ SCFT on $S^{1}$, so $N^{3}$ degrees of freedom are absent at low energy. 
However, at high temperature compared to the inverse-radius of the circle, we expect the 6d CFT physics to be visible, hopefully in our $F$. One subtlety is that fermionic states are counted with minus sign in the index, so there may be cancelation between bosons and fermions. Even after this possible cancelation, we find that the uncanceled free energy still exhibits $N^{3}$ scaling, which proves that the 6d CFT has $N^{3}$ degrees of freedom. We have provided an alternative study of the asymptotic free energy based on $6 \mathrm{~d}$ chiral anomalies, which completely agrees with our D0-D4 calculus.

Our studies based on D0-D4 system also shows that the light D0-brane particles are responsible for the UV enhancement of degrees of freedom. Since D0-branes are the key objects which construct M-theory at strong coupling limit of the type IIA strings, it is natural to see that they are also responsible for the $N^{3}$ degrees of freedom of the $6 \mathrm{~d}(2,0)$ theory. It will be interesting to better understand the single particle index $f\left(\tau, \epsilon_{1,2}, m, v\right)$ which yields this behavior. In particular, conjectures on instanton partons [29] may be addressed in more detail.

The Coulomb branch partition function on $\mathbb{R}^{4} \times T^{2}$ was used as building blocks of interesting CFT indices in the symmetric phase. We comment that our asymptotic free energy proportional to $N^{3}$ does not appear in these symmetric phase indices. Let us explain this with the $6 \mathrm{~d}$ superconformal index, and the DLCQ index.

Firstly, it has been proposed that the D0-D4 partition function, or more precisely this partition function multiplied by the $5 \mathrm{~d}$ perturbative part, is a building block for the 6 d superconformal indices [35-38] on $S^{5} \times S^{1}$. So one might wonder whether our finding $\log Z \propto \frac{N^{3} m^{4}}{\epsilon_{1} \epsilon_{2} \beta}$ (with $\tau_{D}=\frac{i \beta}{2 \pi}$ ) at high temperature has implications to the supercofonrmal index. One can immediately see that the answer is negative. For this discussion, the relevant formula is presented in [38], which uses the product of 3 copies of Coulomb branch partition functions on $\mathbb{R}^{4} \times T^{2}$ as the integrand. The angular momentum chemical potentials of $\mathrm{U}(1)^{2} \subset \mathrm{SO}(6)$ on $S^{5}$ are labeled by three numbers $a_{1}, a_{2}, a_{3}$ satisfying $a_{1}+a_{2}+a_{3}=0$. In this setting, the 3 sets of Omega deformation parameters are given by $\left(\epsilon_{1}, \epsilon_{2}\right)=\left(a_{2}-\right.$ $\left.a_{1}, a_{3}-a_{1}\right),\left(a_{3}-a_{2}, a_{1}-a_{2}\right),\left(a_{1}-a_{3}, a_{2}-a_{3}\right)$ respectively. Since the asymptotic formula for $Z$ is obtained in the limit of small $\epsilon_{1}, \epsilon_{2}$, one can study the superconformal index in the limit of small $a_{1}, a_{2}, a_{3}$. In this limit, the most divergent part in $\epsilon_{1,2}$ is given by

$$
\log Z_{S^{5} \times S^{1}} \sim \frac{N^{3} m^{4}}{\beta}\left[\frac{1}{\left(a_{2}-a_{1}\right)\left(a_{3}-a_{1}\right)}+\frac{1}{\left(a_{3}-a_{2}\right)\left(a_{1}-a_{2}\right)}+\frac{1}{\left(a_{1}-a_{3}\right)\left(a_{2}-a_{3}\right)}\right] .
$$

It is an identity that the sum in the square bracket vanishes, so that the leading asymptotic part proportional to $N^{3}$ vanishes on $S^{5} \times S^{1}$. So our $f_{\text {asymp }}$ has no implication to the superconformal index. However, study of the subleading part $\mathcal{O}\left(\epsilon_{1,2}\right)^{0}$ will be interesting, along the lines of our section 2.2. We hope to come back to this problem in the near future.

Secondly, the M5-brane theory compactified on a lightlike circle can be studied using the D0-D4 quantum mechanics [41, 42]. Its index at DLCQ momentum $k$ can be computed by integrating the D0-D4 index in the Coulomb branch suitably with the Coulomb VEV $v$, as explained in [7]. So one finds (again with $\tau_{D}=\frac{i \beta}{2 \pi} \rightarrow 0$ )

$$
Z_{\mathrm{DLCQ}} \sim \exp \left[-\frac{N^{3} m^{4}}{24 \epsilon_{1} \epsilon_{2} \beta}\right]
$$


Here, unlike the partition function on $\mathbb{R}^{4} \times T^{2}$, where we have notion of multi-particles so that $\log Z$ itself is meaningful as the singe particle index, the DLCQ index is defined with a confining harmonic potential on $\mathbb{R}^{4}[7]$. Thus, the exponent cannot be physically meaningful separately. Also, the definition of $Z_{\mathrm{DLCQ}}$ is such that $\epsilon_{+}=\frac{\epsilon_{1}+\epsilon_{2}}{2}$ has to be real and bigger than other fugacities, as $e^{-\epsilon_{+}}<1$ plays the role of main convergence parameter. So one has to set $\epsilon_{1} \epsilon_{2}>0$. This implies that $Z_{\mathrm{DLCQ}}$ does not exhibit exponential growth, but is rather highly suppressed at small $\beta$, presumably due to boson/fermion cancelation.

From these observations on the superconformal index and the DLCQ index, one realizes that $Z_{\mathbb{R}^{4} \times T^{2}}$ contains interesting dynamical information which may be wiped out in other observables.

Omega deformed partition functions can also be used to study $6 \mathrm{~d}(1,0)$ superconformal field theories. In fact, for many $6 \mathrm{~d}(1,0)$ systems, the index on $\mathbb{R}^{4} \times T^{2}$ is known in the 'self-dual string expansion,' similar to the M-string expansion explained in our section 2.2. The coefficients like $Z_{\left(n_{i}\right)}$ of section 2.2 are elliptic genera of $2 \mathrm{~d}$ CFTs for the $6 \mathrm{~d}$ self-dual strings in the tensor branch. Those elliptic genera have been studied for various $6 \mathrm{~d}(1,0)$ theories [43-48]. The S-duality anomaly and the high temperature asymptotic free energies could be studied using the approaches explored in this paper. This may be an interesting approach to explore the rich physics of $6 \mathrm{~d}$ CFTs and their compactifications to $5 \mathrm{~d} / 4 \mathrm{~d}$.

It would also be interesting to further study the S-duality of the full index of the $(2,0)$ theory, based on some ideas sketched in our section 2.2. Following [26], we find it interesting to study the Wilson/'t Hooft line defects uplifted to 6d surface operators. S-dualities of other defect operators should also be interesting.

Finally, one may ask if a suitable M2-brane partition function on $\mathbb{R}_{\epsilon}^{2} \times S^{1}$ can exhibit $N^{\frac{3}{2}}$ scaling, where $\epsilon$ is the Omega deformation parameter. Although this scaling has been microscopically computed from the $S^{3}$ partition function, or the entanglement entropy, perhaps better physical intuitions can be obtained by directly accounting for where such degrees of freedom come from, like we did for 6d SCFTs on $S^{1}$ from D0-branes (instanton solitons).

\section{Acknowledgments}

We thank Prarit Agarwal, Joonho Kim, Kimyeong Lee, Jaemo Park, Jaewon Song, Shuichi Yokoyama for helpful discussions, and especially Hee-Cheol Kim for many inspiring discussions and comments. We also thank Joonho Kim for helping us with the $\mathrm{SO}(8)$ instanton calculus. This work is supported in part by NRF Grant 2015R1A2A2A01003124 (SK, JN), and Hyundai Motor Chung Mong-Koo Foundation (JN).

Open Access. This article is distributed under the terms of the Creative Commons Attribution License (CC-BY 4.0), which permits any use, distribution and reproduction in any medium, provided the original author(s) and source are credited. 


\section{References}

[1] C. Montonen and D.I. Olive, Magnetic Monopoles as Gauge Particles?, Phys. Lett. B 72 (1977) 117 [INSPIRE].

[2] H. Osborn, Topological Charges for $N=4$ Supersymmetric Gauge Theories and Monopoles of Spin 1, Phys. Lett. B 83 (1979) 321 [INSPIRE].

[3] A. Sen, Dyon-monopole bound states, selfdual harmonic forms on the multi-monopole moduli space and $\mathrm{SL}(2, \mathbb{Z})$ invariance in string theory, Phys. Lett. B 329 (1994) 217 [hep-th/9402032] [INSPIRE].

[4] E. Witten, String theory dynamics in various dimensions, Nucl. Phys. B 443 (1995) 85 [hep-th/9503124] [INSPIRE].

[5] E. Witten, Some comments on string dynamics, in Los Angeles 1995, Future perspectives in string theory, World Scientific, (1996), pp. 501-523, hep-th/9507121 [INSPIRE].

[6] N.A. Nekrasov, Seiberg-Witten prepotential from instanton counting, Adv. Theor. Math. Phys. 7 (2003) 831 [hep-th/0206161] [InSPIRE].

[7] H.-C. Kim, S. Kim, E. Koh, K. Lee and S. Lee, On instantons as Kaluza-Klein modes of M5-branes, JHEP 12 (2011) 031 [arXiv:1110.2175] [INSPIRE].

[8] B. Haghighat, A. Iqbal, C. Kozçaz, G. Lockhart and C. Vafa, M-Strings, Commun. Math. Phys. 334 (2015) 779 [arXiv: 1305.6322] [INSPIRE].

[9] Y. Hwang, J. Kim and S. Kim, M5-branes, orientifolds and S-duality, JHEP 12 (2016) 148 [arXiv: 1607.08557] [INSPIRE].

[10] M. Billó, M. Frau, F. Fucito, A. Lerda and J.F. Morales, S-duality and the prepotential in $\mathcal{N}=2^{\star}$ theories (I): the ADE algebras, JHEP 11 (2015) 024 [arXiv:1507.07709] [INSPIRE].

[11] L. Di Pietro and Z. Komargodski, Cardy formulae for SUSY theories in $d=4$ and $d=6$, JHEP 12 (2014) 031 [arXiv: 1407.6061] [INSPIRE].

[12] R. Flume and R. Poghossian, An algorithm for the microscopic evaluation of the coefficients of the Seiberg-Witten prepotential, Int. J. Mod. Phys. A 18 (2003) 2541 [hep-th/0208176] [INSPIRE].

[13] U. Bruzzo, F. Fucito, J.F. Morales and A. Tanzini, Multiinstanton calculus and equivariant cohomology, JHEP 05 (2003) 054 [hep-th/0211108] [INSPIRE].

[14] C. Hwang, J. Kim, S. Kim and J. Park, General instanton counting and 5d SCFT, JHEP 07 (2015) 063 [Addendum ibid. 04 (2016) 094] [arXiv: 1406.6793] [INSPIRE].

[15] M. Bullimore, H.-C. Kim and P. Koroteev, Defects and Quantum Seiberg-Witten Geometry, JHEP 05 (2015) 095 [arXiv: 1412.6081] [INSPIRE].

[16] M. Bullimore and H.-C. Kim, The Superconformal Index of the (2,0) Theory with Defects, JHEP 05 (2015) 048 [arXiv: 1412.3872] [INSPIRE].

[17] A. Iqbal, C. Kozçaz and K. Shabbir, Refined Topological Vertex, Cylindric Partitions and the U(1) Adjoint Theory, Nucl. Phys. B 838 (2010) 422 [arXiv:0803.2260] [InSPIRE].

[18] R. Gopakumar and C. Vafa, $M$ theory and topological strings. 1., hep-th/9809187 [INSPIRE].

[19] R. Gopakumar and C. Vafa, M theory and topological strings. 2., hep-th/9812127 [INSPIRE].

[20] B. Haghighat, C. Kozçaz, G. Lockhart and C. Vafa, Orbifolds of M-strings, Phys. Rev. D 89 (2014) 046003 [arXiv:1310.1185] [InSPIRE]. 
[21] J. Kim, S. Kim and K. Lee, Little strings and T-duality, JHEP 02 (2016) 170 [arXiv: 1503.07277] [INSPIRE].

[22] M. Billó, M. Frau, L. Gallot, A. Lerda and I. Pesando, Modular anomaly equation, heat kernel and $S$-duality in $N=2$ theories, JHEP 11 (2013) 123 [arXiv:1307.6648] [INSPIRE].

[23] L.F. Alday, D. Gaiotto and Y. Tachikawa, Liouville Correlation Functions from Four-dimensional Gauge Theories, Lett. Math. Phys. 91 (2010) 167 [arXiv:0906.3219] [INSPIRE].

[24] N. Drukker, D. Gaiotto and J. Gomis, The Virtue of Defects in $4 D$ Gauge Theories and 2D CFTs, JHEP 06 (2011) 025 [arXiv: 1003.1112] [INSPIRE].

[25] K. Hosomichi, S. Lee and J. Park, AGT on the S-duality Wall, JHEP 12 (2010) 079 [arXiv: 1009.0340] [INSPIRE].

[26] D. Galakhov, A. Mironov and A. Morozov, S-duality and Modular Transformation as a non-perturbative deformation of the ordinary pq-duality, JHEP 06 (2014) 050 [arXiv: 1311.7069] [INSPIRE].

[27] N. Nemkov, On modular transformations of toric conformal blocks, JHEP 10 (2015) 039 [arXiv: 1504.04360] [INSPIRE].

[28] R. Dijkgraaf, G.W. Moore, E.P. Verlinde and H.L. Verlinde, Elliptic genera of symmetric products and second quantized strings, Commun. Math. Phys. 185 (1997) 197 [hep-th/9608096] [INSPIRE].

[29] B. Collie and D. Tong, The Partonic Nature of Instantons, JHEP 08 (2009) 006 [arXiv:0905.2267] [INSPIRE].

[30] B. Assel, D. Cassani, L. Di Pietro, Z. Komargodski, J. Lorenzen and D. Martelli, The Casimir Energy in Curved Space and its Supersymmetric Counterpart, JHEP 07 (2015) 043 [arXiv: 1503.05537] [INSPIRE].

[31] F. Benini, R. Eager, K. Hori and Y. Tachikawa, Elliptic Genera of $2 d \mathcal{N}=2$ Gauge Theories, Commun. Math. Phys. 333 (2015) 1241 [arXiv:1308.4896] [InSPIRE].

[32] N. Banerjee, J. Bhattacharya, S. Bhattacharyya, S. Jain, S. Minwalla and T. Sharma, Constraints on Fluid Dynamics from Equilibrium Partition Functions, JHEP 09 (2012) 046 [arXiv: 1203.3544] [INSPIRE].

[33] I.R. Klebanov and A.A. Tseytlin, Entropy of near extremal black p-branes, Nucl. Phys. B 475 (1996) 164 [hep-th/9604089] [INSPIRE].

[34] J.A. Harvey, R. Minasian and G.W. Moore, NonAbelian tensor multiplet anomalies, JHEP 09 (1998) 004 [hep-th/9808060] [INSPIRE].

[35] H.-C. Kim and S. Kim, M5-branes from gauge theories on the 5-sphere, JHEP 05 (2013) 144 [arXiv:1206.6339] [INSPIRE].

[36] G. Lockhart and C. Vafa, Superconformal Partition Functions and Non-perturbative Topological Strings, arXiv:1210.5909 [INSPIRE].

[37] H.-C. Kim, J. Kim and S. Kim, Instantons on the 5-sphere and M5-branes, arXiv: 1211.0144 [INSPIRE].

[38] H.-C. Kim, S. Kim, S.-S. Kim and K. Lee, The general M5-brane superconformal index, arXiv: 1307.7660 [INSPIRE]. 
[39] J. Kallen, J.A. Minahan, A. Nedelin and M. Zabzine, $N^{3}$-behavior from $5 D$ Yang-Mills theory, JHEP 10 (2012) 184 [arXiv:1207.3763] [INSPIRE].

[40] N. Bobev, M. Bullimore and H.-C. Kim, Supersymmetric Casimir Energy and the Anomaly Polynomial, JHEP 09 (2015) 142 [arXiv:1507.08553] [INSPIRE].

[41] O. Aharony, M. Berkooz, S. Kachru, N. Seiberg and E. Silverstein, Matrix description of interacting theories in six-dimensions, Adv. Theor. Math. Phys. 1 (1998) 148 [hep-th/9707079] [INSPIRE].

[42] O. Aharony, M. Berkooz and N. Seiberg, Light cone description of (2,0) superconformal theories in six-dimensions, Adv. Theor. Math. Phys. 2 (1998) 119 [hep-th/9712117] [INSPIRE].

[43] J. Kim, S. Kim, K. Lee, J. Park and C. Vafa, Elliptic Genus of E-strings, JHEP 09 (2017) 098 [arXiv: 1411.2324] [INSPIRE].

[44] B. Haghighat, A. Klemm, G. Lockhart and C. Vafa, Strings of Minimal 6d SCFTs, Fortsch. Phys. 63 (2015) 294 [arXiv:1412.3152] [INSPIRE].

[45] A. Gadde, B. Haghighat, J. Kim, S. Kim, G. Lockhart and C. Vafa, 6d String Chains, arXiv: 1504.04614 [INSPIRE].

[46] J. Kim, S. Kim and K. Lee, Higgsing towards E-strings, arXiv:1510.03128 [INSPIRE].

[47] H.-C. Kim, S. Kim and J. Park, 6d strings from new chiral gauge theories, arXiv:1608.03919 [INSPIRE].

[48] M. Del Zotto and G. Lockhart, On Exceptional Instanton Strings, JHEP 09 (2017) 081 [arXiv: 1609.00310] [INSPIRE]. 